\title{
Investigation of spatio-temporal coding in the olfactory bulb of larval Xenopus laevis using fast confocal imaging
}

\author{
PhD Thesis \\ in partial fulfilment of the requirements for the degree \\ Doctor of Philosophy (PhD) in the Neuroscience Program \\ at the Georg August University Göttingen, Faculty of Biology
}

submitted by

Stephan Junek

born in

Potsdam, Germany

December 2008 

Herewith I declare, that I prepared the PhD Thesis 'Investigation of spatiotemporal coding in the olfactory bulb of larval Xenopus laevis using fast confocal imaging' on my own and with no other sources and aids than quoted.

Göttingen, December $8^{\text {th }} 2008$. 

Für Thomas 



\section{CONTENTS}

Table of Contents

vii

List of Figures $\quad$ xi

List of Tables $\quad$ xiii

List of Abbreviations $\quad$ xv

Abstract $\quad$ xvii

$\begin{array}{ll}\text { Acknowledgments } & \text { xviii }\end{array}$

1 Introduction $\quad 1$

1.1 Tasks and Function of Sensory Systems . . . . . . . . . . . . . 1

1.2 The Olfactory System . . . . . . . . . . . . . . 3

1.2.1 Functional organization of the olfactory system . . . . . . 3

1.2.2 Coding principles in the olfactory system . . . . . . . . 9 9

1.3 New Scientific Insights Through New Experimental Tools . . . . . . . 15

1.3.1 Why olfactory research could profit from fast image acquisition 15

1.3.2 Demands on a high-speed fluorescent microscope to study neuronal populations .................... 16

1.3.3 Visualizing neuronal morphology based on fast 3D image acquisition ........................ 17

1.4 Goals of this Thesis . . . . . . . . . . . . . . . 18 
2.1 Electronic Components and Data Acquisition System . . . . . . . . . 19

2.1.1 Control of electronic components . . . . . . . . . . 19

2.1.2 Data acquisition system ................ 20

2.1.3 Tuning and characterization of scan mirror and piezo actuator 22

2.2 Slice Preparation and Physiological Experiments . . . . . . . . . 22

2.2.1 Slice preparation . . . . . . . . . . . . 22

2.2.2 Staining protocols . . . . . . . . . . . . 22

2.2.3 Application of odorants. . . . . . . . . . 24

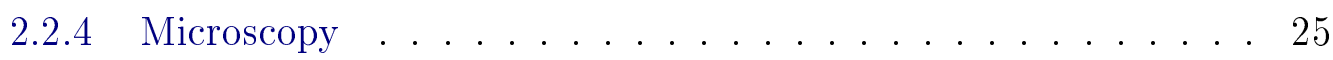

2.2.5 Electrophysiology . . . . . . . . . . . . . 25

2.3 Data Analysis . . . . . . . . . . . . . . . . 26

2.3.1 Characterization of point spread functions . . . . . . 26

2.3 .2 Image analysis . . . . . . . . . . . . . . 26

2.3.3 Autocorrelation map . . . . . . . . . . . 27

2.3.4 Activity correlation imaging ............. 27

$\begin{array}{llr}3 & \text { Results } & 29\end{array}$

3.1 Design, Realization and Characterization of a Fast Confocal Line-

Scanning Microscope . . . . . . . . . . . . . . . 29

3.1.1 Design of the optical pathway . . . . . . . . . . 29

3.1 .2 Spatial resolution . . . . . . . . . . . . . 37

3.1.3 Acquisition speed for 2D- and 3D-imaging . . . . . . . 43

3.1.4 Discussion of the microscope setup . . . . . . . . . . 52

3.2 Activity Correlation Imaging . . . . . . . . . . . . . . . 54

3.2.1 The general principle of activity correlation imaging . . . . . 54

3.2.2 Combining ACI with fast time lapsed 3D imaging . . . . . . 56

3.2 .3 ACI and single cell dye injection . . . . . . . . . . . 61

3.2 .4 Discussion of ACI . . . . . . . . . . . . . 63

3.3 Olfactory Coding with Population Onset Times . . . . . . . . . 66

3.3.1 Measuring odor-evoked responses with high temporal resolution 67

3.3.2 Determination of the response onset time . . . . . . . 69 
3.3.3 Reproducibility and concentration dependence of response on-

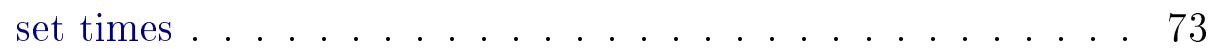

3.3.4 Measures for the similarity of population onset times . . . . 78

3.3.5 Concentration and stimulus dependence of the inversion index $I 83$

3.3.6 Preliminary results ................ 87

$\begin{array}{lll}4 & \text { Discussion } & 97\end{array}$

4.1 Conclusions . . . . . . . . . . . . . . . . . 97

4.2 Future experiments . . . . . . . . . . . . . . . 99

4.3 Response latencies in sensory systems . . . . . . . . . . . . . . 101

4.3.1 Response latencies in the olfactory system . . . . . . . . . 101

4.3.2 Response latencies in other sensory systems . . . . . . . . 102

4.4 Onset patterns and properties of the olfactory system . . . . . . . . 104

4.5 Decoding of response latency patterns . . . . . . . . . . . . 107

$\begin{array}{lll}5 & \text { Summary } & 109\end{array}$

$\begin{array}{lr}\text { Bibliography } & \mathbf{1 1 0}\end{array}$

$\begin{array}{lr}\text { Curriculum Vitæ } & 129\end{array}$

$\begin{array}{ll}\text { List of Publications } & 132\end{array}$ 


\section{List OF FIGURES}

1.1 Organization of the olfactory epithelium . . . . . . . . . 4

1.2 Organization of the olfactory bulb ............. 5

1.3 Larval Xenopus laevis as a model organism for olfactory research . . 8

2.1 Overview of the control system of the scanning microscope . . . . 20

2.2 Schematics of scan protocol. . . . . . . . . . . . . 21

3.1 The microscopic setup of the LIM . . . . . . . . . . . . 30

3.2 Excitation beam pathways . . . . . . . . . . . . 31

3.3 The microscopic setup of the LIM . . . . . . . . . . . . 34

3.4 Point spread function using the $63 \times$ Achroplan $0.95 \mathrm{~W}$. . . . . . . 38

3.5 Response behavior of the scan mirror to voltage steps . . . . . . . . 44

3.6 Response of the scan mirror to ramps at different frequencies and

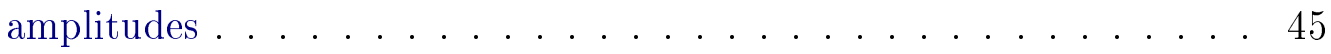

3.7 Response behavior of the piezo actuator to voltage steps . . . . . . . 49

3.8 Response behavior of the piezo actuator at different frequencies . . . 50

3.9 Imaging of spontaneous activity in the olfactory bulb . . . . . . . 55

3.10 Principle of activity correlation imaging . . . . . . . . . . 57

3.11 Activity correlation imaging using fast 3D image acquisition . . . . 58

3.12 Multiple correlation maps using different reference traces . . . . . . 59

3.13 Multi-color representation of the neuronal network . . . . . . . . . 60

3.14 ACI and dye injection . . . . . . . . . . . . . 62

3.15 Scan protocol for odor stimulation experiments . . . . . . . 68

3.16 Determination of the response onset . . . . . . . . . . 70 
3.17 Investigation of latency patterns in the olfactory bulb . . . . . . . 74

3.18 Variability of response onset times . . . . . . . . . . . . 75

3.19 Concentration dependence of response onset times . . . . . . . . . 76

3.20 Inter-neuronal correlations between onset times . . . . . . . . 77

3.21 Two measures for the similarity of latency patterns . . . . . . . . . 79

3.22 Inversion indices for experiments using different concentrations . . . . 84

3.23 Inversion index for experiments using different odorants . . . . . . 86

3.24 Statistical analysis of results . . . . . . . . . . . . 88

3.25 Inversion index for experiments using different complex mixtures . . . 89

3.26 Response onset times in binary mixtures . . . . . . . . . . . . 90

3.27 Concentration dependence of glomerular response onset times . . . . 92

3.28 Strategies for the detection of single action potentials . . . . . . . . 94 


\section{LIST OF TABLES}

3.1 Dimension of illumination line and pixel size . . . . . . . . . 33

3.2 Fit results for the size of point spread functions . . . . . . . . . 41

3.3 Frame acquisition rates for different scan parameters . . . . . . . 46 


\section{List of Abbreviations}

ACI Activity correlation imaging

AP Action potential

FWHM Full width at half maximum

LIM Line illumination microscope

OB Olfactory bulb

ORN Olfactory receptor neuron

$\mathrm{M} / \mathrm{T} \quad$ Mitral/tufted

NA Numerical aperture

PI(D) Proportional - integral (- differential)

PSF Point spread function

ROI Region of interest

Abbreviations exclusively used in figures are explained in the respective figure caption. 


\begin{abstract}
The olfactory bulb (OB) is the only central processing station of the vertebrate olfactory system. It is assumed that odors are represented by a spatio-temporal code in the $\mathrm{OB}$, with both the identity of the activated neurons and the temporal sequence of their activity patterns being stimulus-relevant parameters. An adequate investigation of these coding principles thus relies on the simultaneous recording of a large number of cells with a high temporal resolution. To date, few studies have been based on recordings of this type, and even less have attempted to investigate and quantify aspects of inter-neuronal dependencies in the odor evoked activity patterns. A fast confocal microscope was designed, built, characterized, and subsequently used to study inter-cellular dependencies in neuronal activity patterns, which would be obscured by inter-trial variability in successive single-cell recordings. Specifically, the onset times of odor-evoked activity in ensembles of mitral/tufted cells in the Xenopus larvae $\mathrm{OB}$ were investigated, visualized using the $\mathrm{Ca}^{2+}$ indicator dye Fluo-4. A novel measure, the inversion index, was introduced to quantify the similarity in the order of response onsets between pairs of stimulus applications. Using this measure, it could be shown that these patterns are highly reproducible between applications of a given odor, both for mono-molecular odorants and for complex mixtures. Additionally, the onset order was found to be highly informative about odor identity, while it only relies weakly on the odor concentration. These observations make the onset pattern of $\mathrm{OB}$ neurons a promising candidate for a stimulus-relevant feature of the code implemented in the OB. Relation to other properties of the olfactory system and possible decoding strategies are discussed. In a second application, the fast imaging system was used to acquire time series of image stacks of spontaneous activity in the OB. By exploiting the diverse temporal activity patterns as a means of intrinsic contrast, the individual neurons' dendritic morphology could be visualized. The result of this novel method, termed activity correlation imaging, is a high-contrast multi-color visualization of the neuronal network, as demonstrated on the mitral/tufted cells of the Xenopus larvae OB. Yielding both functional and structural information about neuronal populations, this method opens up unprecedented possibilities for the investigation of neuronal networks.
\end{abstract}




\section{Acknowledgments}

First of all, I would like to thank my supervisor Prof. Schild, for the opportunity to work in his lab for the past years. He gave me an incredible freedom for my work, and was there for me with advice and guidance whenever I needed it.

Many thanks to André Zeug for his inspiration, guidance, amazing patience and for being a great friend during the last years. He helped in ways too numerous to list, from the first drafts of the microscope to the last corrections of this thesis.

Many thanks to Prof. Neher and Dr. Wolf from my PhD committee, who evaluated my work and advised me regularly.

Thanks to Mihai for a great time building the microscope and for being a fun neighbor and a great friend ever since. Your software brought the microscope to life.

I am grateful to Mr. Mesecke, who actually built all the strange shapes that I had come up with, which would eventually be assembled into the microscope. His experience and constant desire to improve and re-invent, but foremost his kindness and modesty made it a great pleasure to work with him. Also many thanks to Mr. Gräbe, and everybody else from the mechanical workshop for their great support whenever I needed it. I always enjoyed coming to your workshop!

I want to thank Thorsten Nägel, Daniel Governatori and Jens Kowalski for help with everything electronic. They were always available and helped immensely in realizing the setup.

Many thanks to Arwed Weigel, with whom I shared the past years of ups and downs. Particularly, thanks for helping me with the setup characterization and, last but not least, for introducing me into the wizardry of making a Feuerzangenbowle. I think we both need one after the submission.

Thanks to Tsai-Wen Chen for working together on the ACI project. I learned a lot during this collaboration.

Thanks to everybody from the lab, Bao, Belle, Dirk, Esther, Eugen, Gudrun, Ivan, Joško, Sebastian, Stefanie and Thomas, for a great atmosphere and advice and support whenever asked for. Special thanks to Esther and Ivan for reading parts of the thesis.

I am grateful to have shared my time here in Göttingen with many wonderful 
people. You are the main reason why I enjoyed these years so much! Thank you, Felipe, Corinna, Alex, Lucian, Alexandra, Foteini, Phil and Michael.

I want to thank all the numerous musicians with whom I played during the last years. You gave me the opportunity to forget everything neuroscience for a couple of hours every week. These were most welcomed breaks.

I want to thank the Göttingen Neuroscience program, and in particular Michael Hörner, Sandra Drube and Simone Cardoso de Oliveira for their great organization.

Many thanks to the Bernstein Center for Computational Biology and to the state of lower saxony, who provided the funding for my work during these years.

My warmest thanks go to my parents, for their unconditional love and support during all my life.

Finally, I want to thank Ioanna, with whom I shared these last years. You gave me the strength to get to this point by distracting me when I was too caught up in work and making me work when I got too distracted, but foremost by always making me smile when I needed it. I will try my best to be as supportive during

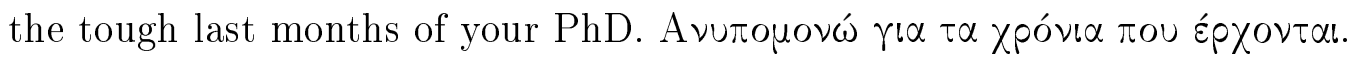




\section{1}

\section{INTRODUCTION}

\section{$1.1 \quad$ Tasks and Function of Sensory Systems}

- In short, our perceptions are not direct records of the world around us. Rather, they are constructed internally according to the constraints imposed by the architecture of the nervous system and its functional abilities.

'Principles of Neural Science', Eric R. Kandel (*1929)

All animals depend on the interaction with their environment. They have to locate food and distinguish it from harmful substances, detect a predator in time, find mating partners, and recognize and protect their offspring. For all these tasks, animals depend on a reliable and immediate knowledge about the world around them. To this end, species have developed a variety of sophisticated sensory systems. Each of these systems captures a certain physical aspect of the environment and translates it into a neural representation. This representation is the basis for a perceptual sensation, an appropriate behavioral response or serves a learning process. The understanding of both the capacity and the function of a sensory system does thus depend on the knowledge of what physical aspects of the environment can be captured by this system, how they are translated into a neural representation, and how this representation on a neuronal or network level relates to the actual sensation that it elicits.

Each sensory system is adjusted to efficiently capture the relevant features of a stimulus space, i.e. the space of all possible values the respective physical quantity can assume. This implies a trade-off between the accuracy set by the sampling 
density and the amount of information the nervous system has to process. Most sensory systems use one of two strategies for an efficient sampling and representation of the respective modality. The first way is to use a small number of functionally specialized receptor types for a sparse sampling of the stimulus space, thus gaining efficiency by sacrificing detail. This strategy is found for example in the five basic tastes of the gustatory system, the three color receptors in the visual system, or the four different receptor types of the somato-sensory system (these numbers refer to the human nervous system, but are similar in other species). Another way is to use a one- or two-dimensional receptor array of identical receptors for a dense sampling of the receptor space. In systems employing this strategy, specificity is derived from the spatial location of the receptor cells. This architecture offers the possibilities of feature classification based on spatial activation patterns and contrast enhancement by local inhibitory networks. These transformations combine the large amount of information contained in the activity patterns of these receptor arrays into a condensed and abstracted representation. At the same time, they maintain and even enhance details contained in the stimulus. Examples for this kind of receptor arrays are found in the retina or the array of hair cells placed along the basilar membrane in the inner ear. Most physical phenomena can be represented with sufficient detail using one of these two strategies or a combination of them (e.g. combining aspects of colors and shapes in the visual system).

The olfactory sense is unique in that it has to cover a very large and heterogeneous stimulus space, which cannot be represented by a two-dimensional map in which the distance of two stimuli is directly related to their degree of difference (Friedrich and Korsching, 1997; Laurent, 2002). This precludes the use of a small number of receptor types, since the sampling of the space would be too sparse. It neither allows a functionally consistent, and architecturally efficient, representation in a oneor two-dimensional odor map. This property of the olfactory system is manifested in the perception of humans. There is no apparent order as found in the pitch of a chromatic scale or the color of the rainbow; there are no fundamental sensations that are perceived independently like the basic tastes of the gustatory system; and there is no direct correlate between the spatial activation patterns and the elicited 
perception as for the recognition of shapes by the visual system. Thus, compared to other sensory systems, we still have a limited understanding of how this sense identifies, categorizes and combines information into the final percept.

\subsection{The Olfactory System}

- Und er roch nicht nur die Gesamtheit dieses Duftgemenges, sondern er spaltete es analytisch auf in seine kleinsten und entferntesten Teile und Teilchen. Seine feine Nase entwirrte das Knäuel aus Dunst und Gestank zu einzelnen Fäden von Grundgerüchen, die nicht mehr weiter zerlegbar waren. Es machte ihm unsägliches Vergnügen, diese Fäden aufzudröseln und aufzuspinnen.

(And he did not merely smell the mixture of odors in the aggregate, but he dissected it analytically into its smallest and most remote parts and pieces. His discerning nose unraveled the knot of vapor and stench into single strands of unitary odors that could not be unthreaded further. Unwinding and spinning out these threads gave him unspeakable joy.)

‘Das Parfüm', Patrick Süskind (*1949)

The olfactory system was among the last of the sensory systems for which the basic principles of transduction and coding were understood, with many details still being highly debated today (Fletcher et al., 2005; Mombaerts, 2004). In fact, while the molecular basis of odor sampling is mostly agreed upon, the means of information representation and processing in the olfactory bulb, which are important for odor learning, discrimination and identification, are active areas of research with a variety of proposed hypotheses, but without a common consensus to date (for reviews, see (Friedrich, 2006; Laurent, 2002; Lledo et al., 2005)).

\subsubsection{Functional organization of the olfactory system}

\section{The olfactory epithelium}

The detection of on odor starts in the olfactory epithelium, a sheet of cells covering the nasal cavity. The olfactory epithelium is composed of three cell types (Fig. 1.1): the olfactory receptor neurons (ORNs) are responsible for the transduction process and the relay of action potentials into the olfactory bulb (OB); the sustentacular cells are glia-like supporting cells (Getchell, 1977; Okano and Takagi, 1974); the 


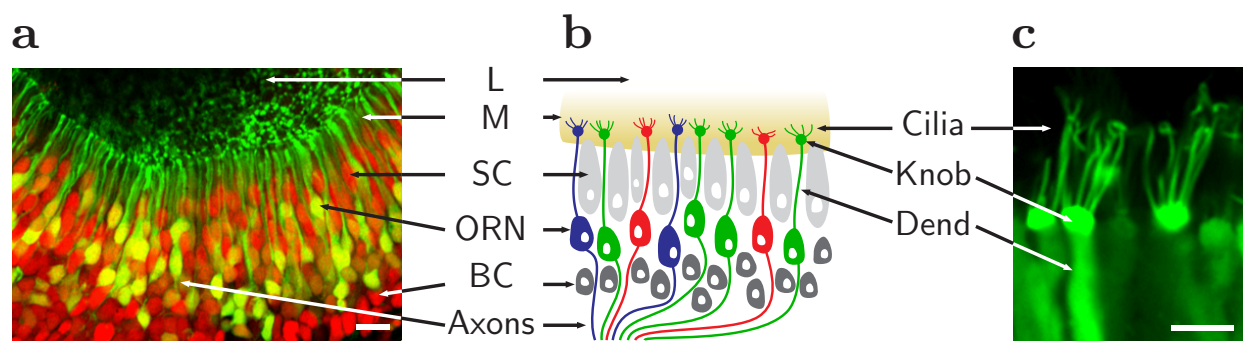

Figure 1.1: Organization of the olfactory epithelium. The olfactory epithelium consists of three cell types: the olfactory receptor neurons; the sustentacular cells, which are glia-like supporting cells; and the basal cells, which provide the epithelium with the capacity of cell regeneration. (a) Staining of the olfactory epithelium of a Xenopus laevis tadpole, adopted from Manzini et al. (2002). The ORNs were backfilled through the nerve using the biocytin/avidin staining (green), the slice was counterstained with propidium iodide (red). Scale bar, $20 \mu \mathrm{m}$. (b) Schematic representation of the olfactory epithelium. Each ORN expresses a single type of olfactory receptor, indicated here by different colors. The receptors are located in the membrane of the cilia, which extend into the lumen and are covered by a layer of mucus. The axons of the ORNs are projecting to the olfactory bulb through the olfactory nerve. (c) Magnification of the dendritic terminal, termed the dendritic knob, which is decorated with numerous cilia. This is the place of odor binding, that initiates the odor recognition process. Adopted from Manzini et al. (2002). Scale bar, $5 \mu \mathrm{m}$.

Abbreviations L: lumen of the nasal cavity; M: mucus; SC: sustentacular cell; ORN: olfactory receptor neuron; BC: basal cell; Dend: dendrite.

basal cells provide the epithelium with the capacity of cell regeneration (Graziadei, 1971). The ORNs have a large dendrite projecting towards the lumen of the nasal cavity. The dendrite terminates in the dendritic knob, a protrusion decorated with a number of fine cilia extending into the lumen (Fig. 1.1). These cilia contain the olfactory receptors, which are responsible for binding of odorous molecules. The olfactory receptors form a family of seven-transmembrane proteins belonging to the group of G-protein coupled receptors (Buck and Axel, 1991). The specificity of odor recognition is achieved by a differential expression of olfactory receptors in the ORNs. From the large receptor family (up to about 1,000 in higher vertebrates (Buck and Axel, 1991; Mombaerts, 1999), about 400 in the frog (Niimura and Nei, 2006)) typically a single receptor is expressed in each ORNs (Buck, 1996). Each of 
a

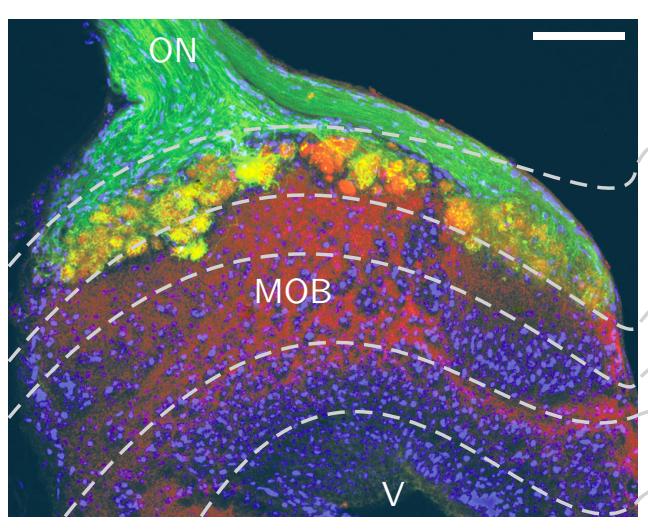

b

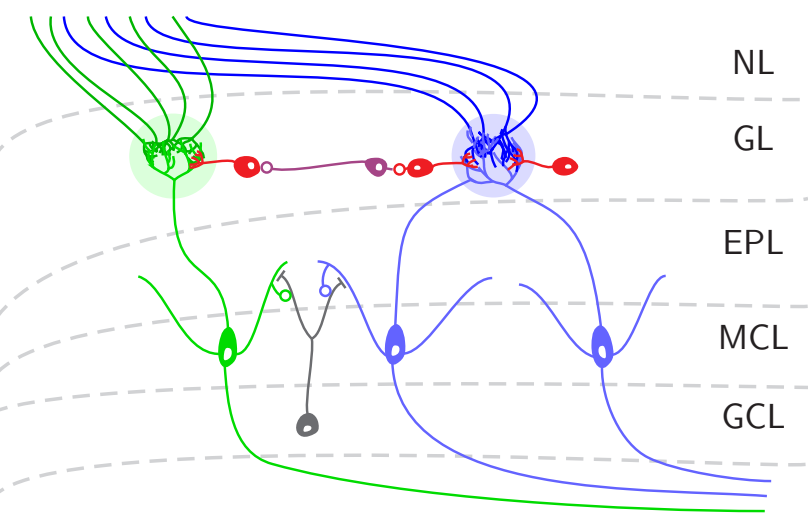

Figure 1.2: Organization of the olfactory bulb. The OB is a layered structure, consisting of the nerve layer, the glomerular layer, the external plexiform layer, the mitral cell layer, and the granule cell layer. Refer to the text for a summary of the anatomy and connectivity of the OB. (a) Triple staining of the OB of a Xenopus laevis tadpole, adopted from Nezlin et al. (2003). Biocytin injection into the olfactory nerve (green), synaptophysin immunostaining (red), and DAPI nucleic acid staining (blue). Scale bar, $100 \mu \mathrm{m}$. (b) Schematic representation of the OB. The axons of the ORNs converge in a receptor specific manner (indicated by colors) in the olfactory glomeruli. $\mathrm{M} / \mathrm{T}$ cells are the principle neurons of the vertebrate OB. They send their primary dendrite into the glomeruli, where they receive input from the ORNs and they send their axons to higher brain regions. Several types of local interneurons modulate the $\mathrm{M} / \mathrm{T}$ cell activity. The $\multimap-$ symbol indicates excitatory, the $\dashv$-symbol inhibitory synapses. Refer to the text for details.

Abbreviations ON: olfactory nerve; MOB: main olfactory bulb; V: ventricle; NL: nerve layer; GL: glomerular layer; EPL: external plexiform layer; MCL: mitral cell layer; GCL: granule cell layer.

these receptors can typically bind a variety of odor molecules with different affinities, resulting in odor specific activation patterns in the olfactory epithelium (DuchampViret et al., 1999; Kajiya et al., 2001; Malnic et al., 1999; Manzini and Schild, 2004).

\section{The olfactory bulb}

The OB receives information from the olfactory epithelium through the axons of the ORNs. It is the first and only central relay station of the olfactory system, since its projections diverge into various parts of the brain (Kandel et al., 2000). Accordingly, 
much effort has been directed during the last decades towards uncovering the coding strategies implemented by the OB.

The OB has an anatomically layered structure consisting of the nerve layer, the glomerular layer, the external plexiform layer, the mitral cell layer and the granule cell layer (Fig. 1.2). The axons of the ORNs terminate in large neuropil structures known as the olfactory glomeruli, which are located in the periphery of the OB. The ORNs show a specific convergence pattern, with the axons of neurons expressing the same receptor converging typically into two olfactory glomeruli (Ressler et al., 1994; Vassar et al., 1994). In this way, the specificity achieved by the differential receptor expression in hundreds of thousands of individual ORNs is not only maintained but condensed into a spatial map with only two elements per receptor type. This map is however different from the topographical maps found in other sensory systems (Kandel et al., 2000), as there is no consistent functional pattern evident in the spatial arrangement of this 'glomerular map' (Friedrich and Korsching, 1997; Lledo et al., 2005; Meister and Bonhoeffer, 2001).

The mitral/tufted $(\mathrm{M} / \mathrm{T})$ cells are the principle neurons of the $\mathrm{OB}$, they receive input from the receptor neurons and their axons project to higher brain regions. Their morphology and their connectivity with other neurons provide the possibility of processing the incoming 'raw' signal into the outgoing olfactory code. Each M/T cell has one apical dendrite that extends into the glomerular layer. At its end, it branches extensively, forming a glomerular tuft in typically a single glomerulus (Lledo et al., 2005), though innervation of several glomeruli by the same M/T cell have been reported (Nezlin et al., 2003). Additionally, M/T cells possess several lateral dendrites which extend into the external plexiform layer, and which can span almost the complete olfactory bulb (Mori et al., 1983; Orona et al., 1984). The following connectivity patterns have been found to date in the OB and are thought to be important for the olfactory coding (for a thorough review see Lledo et al. (2005)):

1. The glomerular tufts of both M/T (Berkowicz et al., 1994) and periglomerular (Bardoni et al., 1996) cells receive excitatory (glutamatergic) input from the axon terminals of the ORNs. These can act via NMDA and AMPA receptors 
(Aroniadou-Anderjaska et al., 1997; Bardoni et al., 1996; Berkowicz et al., 1994; Ennis et al., 1996).

2. The $\mathrm{M} / \mathrm{T}$ cells innervating the same glomerulus are inter-connected by electrical synapses (Christie et al., 2005; Paternostro et al., 1995).

3. The glomerular tufts of $\mathrm{M} / \mathrm{T}$ cells are innervated by dendrites of a variety of juxtaglomerular cells. These cells form both intra- and interglomerular connections and have been suggested to form a center-surround inhibitory network at the glomerular level in the mouse (Aungst et al., 2003).

4. Another presumed center-surround network is formed by the axon-less, GABAergic inhibitory granule cells which make contact with the secondary dendrites of M/T cells (Shipley and Ennis, 1996).

5. M/T cells form dendrodendritic synapses with granule cells (Pinching and Powell, 1971; Rall et al., 1966), providing a mechanism of $\mathrm{M} / \mathrm{T}$ cell selfinhibition (Isaacson and Strowbridge, 1998; Jahr and Nicoll, 1982) which appears to depend on NMDA receptors (Schoppa et al., 1998). Dendrodendritic synapses are also the main connection between juxtaglomerular cells (Pinching and Powell, 1971).

6. Various cases of self-modulation are found in the OB (Isaacson, 1999; Nicoll and Jahr, 1982). These were reported to be involved in driving slow oscillations of the membrane potential (Schoppa and Westbrook, 2001), helping to synchronize M/T cells that project to the same glomerulus (Schoppa and Westbrook, 2002), and supporting action potential backpropagation (Salin et al., 2001). While all these phenomena depend on self-excitation of $\mathrm{M} / \mathrm{T}$ cells, selfinhibition has been reported for periglomerular cells (Smith and Jahr, 2002).

\section{Olfactory systems across species and the choice of a model organism}

Many properties of the olfactory system are very conserved across species from nematodes to mammals (for reviews, see Ache and Young (2005); Eisthen (2002); Hildebrand and Shepherd (1997)). Specifically, investigations of insects (Hansson 
a

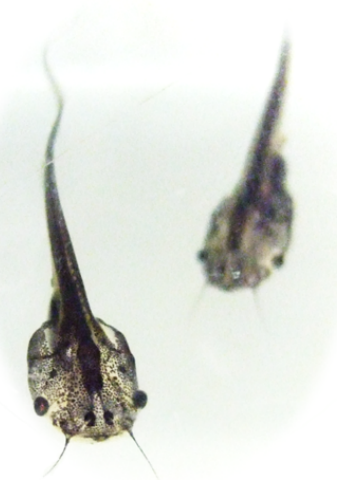

b

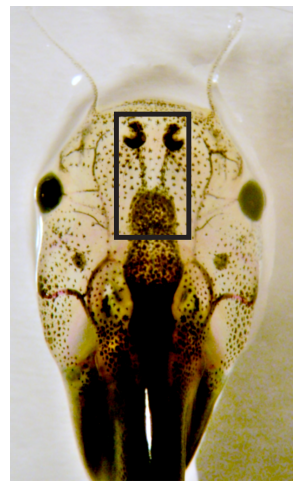

C

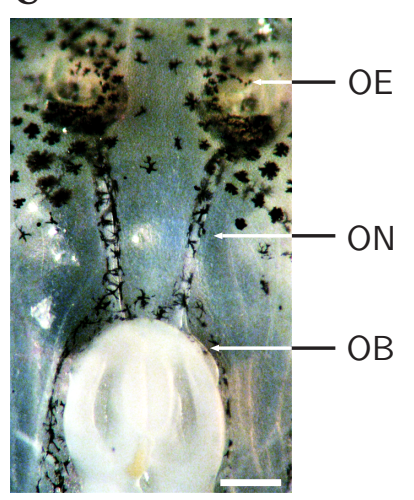

Figure 1.3: Larval Xenopus laevis as a model organism for olfactory research. (a) Larval Xenopus laevis. (b) Head of a Xenopus laevis tadpole. The outline indicates the tissue block that is cut out for the nose-brain preparation. (c) Slice of the tissue block indicated in (b), containing the olfactory epithelia, the olfactory nerves and the olfactory bulbs. This preparation is termed nosebrain preparation and is used for the physiological experiments throughout the thesis. Scale bar, $500 \mu \mathrm{m}$. Photographs kindly provided by T. Hassenklöver (a) and S. Gliem (b,c).

Abbreviations OE: olfactory epithelium; ON: olfactory nerve; OB: olfactory bulb.

and Anton, 2000; Stocker, 2001), amphibians (Manzini and Schild, 2004; Nezlin and Schild, 2000; Nezlin et al., 2003), fish (Byrd and Brunjes, 1995; Korsching et al., 1997), and mammals (Shipley and Ennis, 1996) indicate, that the main features of the olfactory epithelium (differential expression of olfactory receptors in ORNs) and the olfactory bulb or its analogous structures (ORN axons converge in an receptor specific manner in olfactory glomeruli; a single layer of principle neurons; inhibitory interneurons both on the level of glomeruli and of principle neurons) are shared among insects and different classes of vertebrates. This high degree of structural similarity suggests that there might be also similarities with respect to the olfactory code that is created by the underlying network. Consequently, a wide variety of model organisms from different classes have contributed to the current understanding of the olfactory system.

The ultimate goal of neuroscientific research is the understanding of the human nervous system, yet most neurophysiological research is conducted using animal 
models. Besides ethical considerations, these model systems often have the advantage of being less complex, while exhibiting the same fundamental features as higher evolved species. Furthermore, some species have properties that facilitate experimental research, such as short reproduction times, easy manipulation of the gene code, transparency of the tissue or simply small size. The choice of an appropriate model organism is thus in many cases the key to successful research.

The tadpole of Xenopus laevis (Fig. 1.3) has proven to be an excellent model system for investigations both of the olfactory epithelium (Hassenklöver et al., 2008; Manzini and Schild, 2003a, 2004) and of the olfactory bulb (Czesnik et al., 2001, 2003; Manzini et al., 2007a; Nezlin and Schild, 2005). The major experimental advantage of this animal is the lack of the cribriform plate, the bone separating the nasal cavity and the olfactory bulb. It is thus easy to create a 'nose-brain' preparation that can be used to study the effects of natural odors in the OB (Fig. 1.3c, Czesnik et al. (2003)). The olfactory system of tadpoles is known to be functional and behaviorally relevant (Kiesecker et al., 1996; Waldman, 1985), justifying the use of an embryonic animal for the functional investigation of a sensory system. Furthermore, it is known that amino acids are behaviorally relevant stimuli for Xenopus laevis tadpoles (Kiseleva, 1983, 1995), as they are for other aquatic animals (Carr, 1988; Ferrer and Zimmer, 2007). Amino acids form a convenient set of stimuli, as they are chemically well defined, and encompass both similar and dissimilar molecules. Consequently, many studies of the olfactory system of aquatic animals have used amino acids for odor stimulation (Czesnik et al., 2003; Friedrich and Korsching, 1998; Manzini et al., 2007b, 2002; Tabor et al., 2004).

\subsubsection{Coding principles in the olfactory system}

\section{Processing of information in the olfactory bulb}

In all sensory systems, the information captured by the receptor cells is processed on its way to higher brain centers. The investigation of these processes in different sensory systems revealed recurrent mechanisms, the most famous probably being the concept of local contrast enhancement by lateral inhibition, first discovered in the retina (Hartline, 1969). This processing of the sensory information serves two 
main purposes: to locally enhance contrast in order to emphasize differences between similar activation patterns; and to combine aspects of the activity patterns into more abstracted representations that allows a categorization of the captured stimulus.

Single molecules usually activate a variety of receptor types (Duchamp-Viret et al., 1999; Kajiya et al., 2001; Malnic et al., 1999; Manzini and Schild, 2004). This observation led to the conclusion that a given odor (which is in most cases a complex mixture of different molecules) is characterized by the specific combination of activated olfactory receptors. This strategy of odor coding is referred to as 'population coding'. The pattern of activated ORNs is transformed into a pattern of activated $\mathrm{M} / \mathrm{T}$ cells by the circuits in the $\mathrm{OB}$ described above. While much is known about the anatomical and molecular details of these circuits, the functional significance of this transformation is still debated. The problems of uncovering the coding strategies largely originate from the complexity of the odor space, and the difficulty to define odor categories and a measure for odor similarity.

It has been proposed that the lateral connections formed at the level of glomeruli by the juxtaglomerular cells (Aungst et al., 2003) and at the $\mathrm{M} / \mathrm{T}$ level by the granule cells (Shipley and Ennis, 1996) serve the function of local contrast enhancement (Aungst et al., 2003; Johnson et al., 1999; Vucinić et al., 2006; Yokoi et al., 1995), in analogy to other sensory systems (Hartline, 1969; Kandel et al., 2000). However, only one of these studies provided evidence for center-surround receptive fields of M/T cells (Yokoi et al., 1995). Furthermore, the role of the center-surround architecture for sharpening of tuning curves has been questioned (Fantana et al., 2008; Urban, 2002), including suggestions for alternative roles, such as the removal of concentration dependency of the activity patterns (Cleland et al., 2007). The significance of lateral interactions for odor discrimination and recognition tasks has additionally been challenged by the observation that ablation of large parts of the OB do not impair odor discrimination abilities (Bisulco and Slotnick, 2003; Fecteau and Milgram, 2001; Slotnick et al., 1987). Consequently, the precise role of the OBs' interneurons and of its characteristic connectivity pattern is still unknown.

Furthermore, while most sensory systems show a topographical organization of neurons at different levels, the $\mathrm{M} / \mathrm{T}$ cells are not organized in such a spatio-functional 
way. It has been shown recently, that $\mathrm{M} / \mathrm{T}$ cells innervating the same glomerulus are both highly synchronous in their activity but also spread across most of the OB (distances up to half the bulb diameter, Chen et al., submitted). The spatial map of activated $\mathrm{M} / \mathrm{T}$ cells is thus not informative about the stimulus, and one should rather use a term like 'identity map' to refer to the functional activity pattern of $\mathrm{M} / \mathrm{T}$ cells. For the sake of consistency, the former term is retained throughout this thesis.

\section{Spatio-temporal coding strategies}

While it is unquestioned that the combinatorial activity patterns of $\mathrm{M} / \mathrm{T}$ cells carry information about the stimulus, it has been hypothesized that odor identification and discrimination requires more information than the identity of the activated $\mathrm{M} / \mathrm{T}$ cells. In fact, some of the earliest investigations of OB neurons concerned the temporal patterning of spontaneous and odor-evoked activity (Adrian, 1942).

Walter J. Freeman later proposed a role of chaotic dynamics in the OB for olfactory coding. This theory was based on the complex temporal structure found in olfactory electroencephalograms (Freeman, 1991, 1994; Skarda and Freeman, 1987). These recordings had the disadvantage of averaging over larger populations of neurons, which proved to be unsuitable for the investigation of the olfactory system.

More recent models were motivated by the reproducibility and specificity of temporal activity patterns recorded from individual $\mathrm{M} / \mathrm{T}$ cells. These models were also considering two other properties of the olfactory system: (1) The M/T cells show clear temporal patterning of their activity in response to odor stimulation. (2) Smell is a sense with a comparably low temporal resolution, which allows the use of time as a coding parameter (Laurent, 1999). The two most influential approaches will be introduced briefly.

The model proposed and developed by Gilles Laurent (for a review, see Laurent et al. (2001)) is based on two observations: (1) During odor stimulation, projection neurons and local neurons (the insect analogs to $\mathrm{M} / \mathrm{T}$ cells and granule cells, respectively) exhibit sub-threshold oscillations $(20-30 \mathrm{~Hz})$ of their membrane potential. These oscillations are phase-locked for all projection neurons and have a 
fixed phase-shift of $90^{\circ}$ between projection neurons and local neurons. It is possible to measure these oscillations by extracellular recordings of the local field potential. The oscillations cannot be found in ORNs, and are thus attributed to the circuitry of the antennal lobe (the insect analog to the OB). (2) The odor-evoked responses of projection neurons are characterized by cell- and odor-specific temporal patterns of increased and decreased activity. The timing of action potentials relative to the local field potential oscillations depends on the cell, the stimulus and the phase of the response, creating a reproducible sequence of phase-locked and non-phase-locked epochs during the evoked response. According to this model, an understanding of the olfactory code relies on a combined analysis of the local field potentials oscillations and the spike pattern of the individual projection neurons. It was further shown that the temporal activity patterns become more odor specific over the course of the evoked response, resulting in a gradually optimized stimulus representation (Friedrich and Laurent, 2001). This was shown by analyzing the degrees of correlation between the $\mathrm{M} / \mathrm{T}$ cell activity pattern and by performing principle component analysis in $200 \mathrm{~ms}$ time windows for a variety of odorants. These analyses suggest that odor classification is best performed in the initial $600 \mathrm{~ms}$ of the response, during which similar odors elicit similar excitation patterns. During the later phase of the response, the patterns created by chemically similar odors become more distinct, facilitating odor identification. Another extension of the model uses a classification of phase-locked vs. non-phase-locked spikes, suggesting that these two populations of spikes carry distinct information about odor category and odor identity, respectively (Friedrich et al., 2004).

John J. Hopfield approached the question of olfactory coding from a more computational and information theoretical point of view, using properties of actual biological systems as an inspiration for rather general models for sensory coding. He proposed a variety of mechanisms that could play a role for olfactory coding, including the analysis of temporal fluctuations in stimulus intensity (Hopfield, 1991); the timing of action potentials relative to a global underlying oscillation (Hopfield, 1995); the role of differential activation of a large number of receptor types (Hopfield, 1999); and the use of synchronized subpopulations of $\mathrm{M} / \mathrm{T}$ cells for olfactory 
coding. Similar to the model proposed by Laurent, a global oscillation that serves as a temporal reference is an important aspect of Hopfield's models (Hopfield, 1995; Hopfield and Brody, 2004). However, while Hopfield only considers action potential timing relative to this oscillation, Laurent explicitly exploits the evolution of temporal $\mathrm{M} / \mathrm{T}$ cell dynamics over hundreds of milliseconds.

\section{Time scales set by behavioral discrimination tasks}

The described studies have significantly advanced our understanding of the OB dynamics. However, the coding strategies proposed by Hopfield are only loosely based on the actual olfactory system and have to be validated experimentally. The model proposed by Laurent, on the other hand, demands time scales for odor discrimination around $1 \mathrm{~s}$, which is significantly longer than the reaction times observed in most sensory systems, including the olfactory system. Odor discrimination tasks have been performed in humans and rodents. In rodents, different discrimination tasks have been tested, using single odors and binary mixtures (Abraham et al., 2004), odors with a varying degree of similarity (Slotnick, 2007b; Uchida and Mainen, 2003) and discrimination between novel and familiar odors (Wesson et al., 2008). While there are subtle differences in the results, the overall discrimination times range typically between 200 and $400 \mathrm{~ms}$, with the exception of tasks concerning the discrimination of novel odors (Slotnick, 2007a). The response times measured for humans are slightly longer (around $400 \mathrm{~ms}$, Laing (1986)), but the results are similar to the studies in rodents in that the odor identification can be achieved within a single sniff (Abraham et al., 2004; Uchida and Mainen, 2003). The response times measured in these discrimination tasks do not only rely on the processing of information in the OB, but they include decision making and initiation of the actual motor response. This implies that the time interval used for the 'discrimination analysis' is even shorter than the reported response times. As the coding strategy proposed by Laurent et al. is based on studies in insects and fish, and the behavioral studies were conducted in higher vertebrates, a direct comparison between their results is not possible. Nevertheless, the behavioral experiments indicate that odor identification and discrimination might not depend on the changes in neuronal ac- 
tivity over almost one second, but that the relevant information is contained in the activity patterns over shorter time scales. In order to investigate the odor specific variations in the population dynamics shortly after stimulus application, it would be required to observe a significant $\mathrm{M} / \mathrm{T}$ cell population with a high temporal resolution. The variability of temporal activity patterns within short time intervals (few hundred milliseconds) is limited, especially considering the stereotypic bursting found in M/T cells in various species (Margrie and Schaefer, 2003; Scheidweiler et al., 2001; Wellis et al., 1989). It is thus reasonable to investigate concrete parameters of the responses, such as the response latency, i.e. the time between odor onset and the first spike, or the mean firing rate. This approach is further supported by recent findings in other sensory systems, that indicate a role of response latencies for rapid and reliable coding of sensory information (Carr and Konishi, 1990; Gollisch and Meister, 2008; Johansson and Birznieks, 2004; Reich et al., 2001). First attempts in this direction have been made in the olfactory system, either using electrophysiological tools (Bathellier et al., 2008; Margrie and Schaefer, 2003), or functional imaging (Spors and Grinvald, 2002; Spors et al., 2006). The electrophysiological recordings suffer from the low number of simultaneously recorded neurons, while the imaging experiments were investigating olfactory glomeruli, rather than $\mathrm{M} / \mathrm{T}$ cells. Most importantly, none of these studies tried to quantitatively evaluate the observed parameters under a population coding paradigm. Such an approach requires a trial-by-trial population analysis, instead of first averaging over trials for single cells and subsequently investigating the population dynamics. 


\subsection{New Scientific Insights Through New Experimental Tools}

- Misura ciò che è misurabile, e rendi misurabile ciò che non lo è. 9

(Measure what can be measured, and make measurable what can not.) probably Galileo Galilei (1564 - 1642)

\subsubsection{Why olfactory research could profit from fast image acquisition}

Our understanding of the nervous system, and of nature in general, was always advanced by the introduction of novel experimental tools. The advent of molecular biology in the second half of the $20^{\text {th }}$ century has provided powerful tools that enabled the uncovering of the molecular principles underlying the early stages of olfactory coding (Buck and Axel, 1991; Ressler et al., 1994; Vassar et al., 1994). Significant improvements in photochemistry on the other hand provided functional dyes, such as voltage or calcium sensitive fluorophores (Orbach et al., 1985; Tsien, 1981), which are today ubiquitously used to monitor neuronal activities in large populations of cells. Thanks to confocal and multi-photon microscopy, it is possible to measure fluorescent signals with high specificity in all three spatial dimensions, even deep inside living tissue (Stosiek et al., 2003; Yaksi et al., 2007).

These advances allow the simultaneous observation of the activity of a large number of neurons with a high spatial resolution. They suffer however from a low temporal resolution, usually at the order of a few Hertz for both conventional confocal and widefield microscopes. The time scale at which neurons communicate is about a hundred times shorter. The investigation of functional aspects of neuronal systems is thus limited to either electrophysiological recordings with high temporal, but effectively no spatial resolution, or to the observation of neuronal populations using imaging systems with a low temporal resolution. Since the olfactory system is characterized by population coding, the investigation of a single or a small number of cells can yield only an incomplete understanding of the olfactory coding strategies. This motivates the investigation of odor-evoked population responses with a high temporal resolution. First attempts in this direction have been made (Spors and Grinvald, 2002; Spors et al., 2006), though with the drawback of a low spatial res- 
olution, which restricted the experiments to the observation of olfactory glomeruli, which are about three times larger than neuronal somata. Since the M/T cells are the neurons projecting to higher brain areas, it is of particular interest to investigate the activity patterns of these cells. This requires a microscopic tool with high spatial and high temporal resolution.

\subsubsection{Demands on a high-speed fluorescent microscope to study neu- ronal populations}

A microscope designed for the investigation of neuronal populations with high temporal resolution should meet the following requirements:

1. The spatial resolution has to be sufficient to distinguish single somata in all three spatial dimensions. This implies the ability of creating so called 'optical sections', i.e. to exclude out-of-focus light, as realized by the confocal microscope first introduced by Marvin Minsky (Minsky, 1957, 1988).

2. The temporal resolution should be sufficient to capture details of neuronal activity patterns. The time scale at which neurons communicate is in the range of milliseconds, which defines the desired resolution for a fast microscope.

3. Since biological systems show large inter-trial variability, it is required to repeat a given experiment several times in the same preparation, in order to separate systematic from random events.

These considerations motivated the design of a novel confocal microscope optimized for fast imaging of biological specimen. By focusing the light into a line instead of a point as in conventional laser scanning microscopes, the scanning is reduced to one dimension, and in combination with a fast detector high frames rates can be achieved. A number of line scanning realizations have been described (Im et al., 2005; Masters and Thaer, 1994; Sheppard and Mao, 1988), most often though with applications to non-fluorescent samples or non-biological specimens. The new microscope was thus designed to (1) maximize efficiency in the emission pathway, (2) optimize the trade-off between spatial resolution and signal-to-noise ratio for imag- 
ing of neuronal populations, and (3) provide the possibility to restrict fast image acquisition to short time intervals of interest.

\subsubsection{Visualizing neuronal morphology based on fast $3 \mathrm{D}$ image acquisi- tion}

While the microscope setup was primarily motivated by questions concerning olfactory coding, its application is not limited to fast 2D imaging. By extending the image acquisition to the third dimension, considerably larger fractions of a network could be observed, and the analysis could be extended from somata to neuronal processes. In this way, another important aspect of the study of the brain could be approached, namely the investigation of the structure of neuronal networks. The density of biological tissues generally requires a sparse staining in order to generate images with sufficient contrast for the visualization of individual neuron's morphology. Instead of using the fluorescent intensities for the generation of image contrast, it is possible to exploit other parameters, as it is commonly done in functional MRI. In the case of neuronal networks, the complex and diverse temporal structure of neuronal activity, visualized by a calcium indicator dye, could be exploited as a means of intrinsic contrast. By using fast image acquisition, it is possible to observe a large fraction of a network quasi simultaneously. Based on these recordings, the spatial positions exhibiting a given activity pattern can be detected by means of correlation analysis. This approach should enable the detection of functionally synchronous structures in the volume under observation.

This section of the thesis was a collaboration with Tsai-Wen Chen, Department of Neurophysiology and Cellular Biophysics, University Göttingen. 


\subsection{Goals of this Thesis}

This thesis aims at deepening our understanding of function and structure of the olfactory system, in particular the spatio-temporal coding in the olfactory bulb. To shed new light on this yet unresolved problem, novel experimental and analytical tools were designed, implemented and used. The presented work encompasses three successive parts:

1. A novel confocal microscope for fast 2- and 3-dimensional image acquisition was designed and realized. The microscope was characterized concerning its spatial and temporal resolution. This characterization was the basis for the subsequent biological applications.

2. A novel way of visualizing the architecture of neuronal networks from time series of functional 3D-imaging data was developed. By acquiring complete image stacks with sub-second resolution with the new microscope, large parts of a network could be imaged quasi simultaneously. The introduced algorithm generates a high-contrast multi-color visualization of the network, which complements the functional imaging data for an unprecedented combination of structural and functional information about the network under observation.

3. The early phase of the odor-evoked response in the OB of Xenopus laevis tadpoles was measured with high temporal resolution, taking advantage of the fast 2D-imaging capabilities of the new microscope. These recordings were used to investigate aspects of olfactory coding, in particular the population patterns of $\mathrm{M} / \mathrm{T}$ cell response onset times. 


\section{2}

\section{Materials AND Methods}

\subsection{Electronic Components and Data Acquisition System}

The novel line-illumination microscope contains a number of electronic components, such as the CCD camera, the scan mirror and the piezo actuator. It depends therefore on a system to control and synchronize these elements. Additionally, a synchronization with external devices, such as a stimulus application system or a patch clamp unit is desirable.

\subsubsection{Control of electronic components}

The control of the electronic components and the data acquisition was distributed to two PCs to avoid conflicts of these demanding processes. The control unit PC is the 'master unit' of the setup (Fig. 2.1). It creates the scan schemes for the scan mirror and the piezo actuator as well as the trigger for the image acquisition, controls the shutter, the neutral density filters, and provides an external trigger to synchronize the image acquisition to other instruments (e.g. a patch clamp setup or an stimulus application system). These processes are controlled and interfaced using the software linlab custom written in $\mathrm{C}++$ by Mihai Alevra (Department of Neurophysiology and Cellular Biophysics, University Göttingen). The user interface enables the creation of standard scan protocols, resulting in scan schemes as shown in Figure 2.2, including the specification of pre-acquisition lines and pre-acquisition frames to exclude the 'fly-back' times of the scan mirror and the objective from the image acquisition. More complex scan protocols can be created and exported using an interface written for MATLAB by Stephan Junek, the exported files can then be loaded into linlab. This software allows for example the change of temporal 


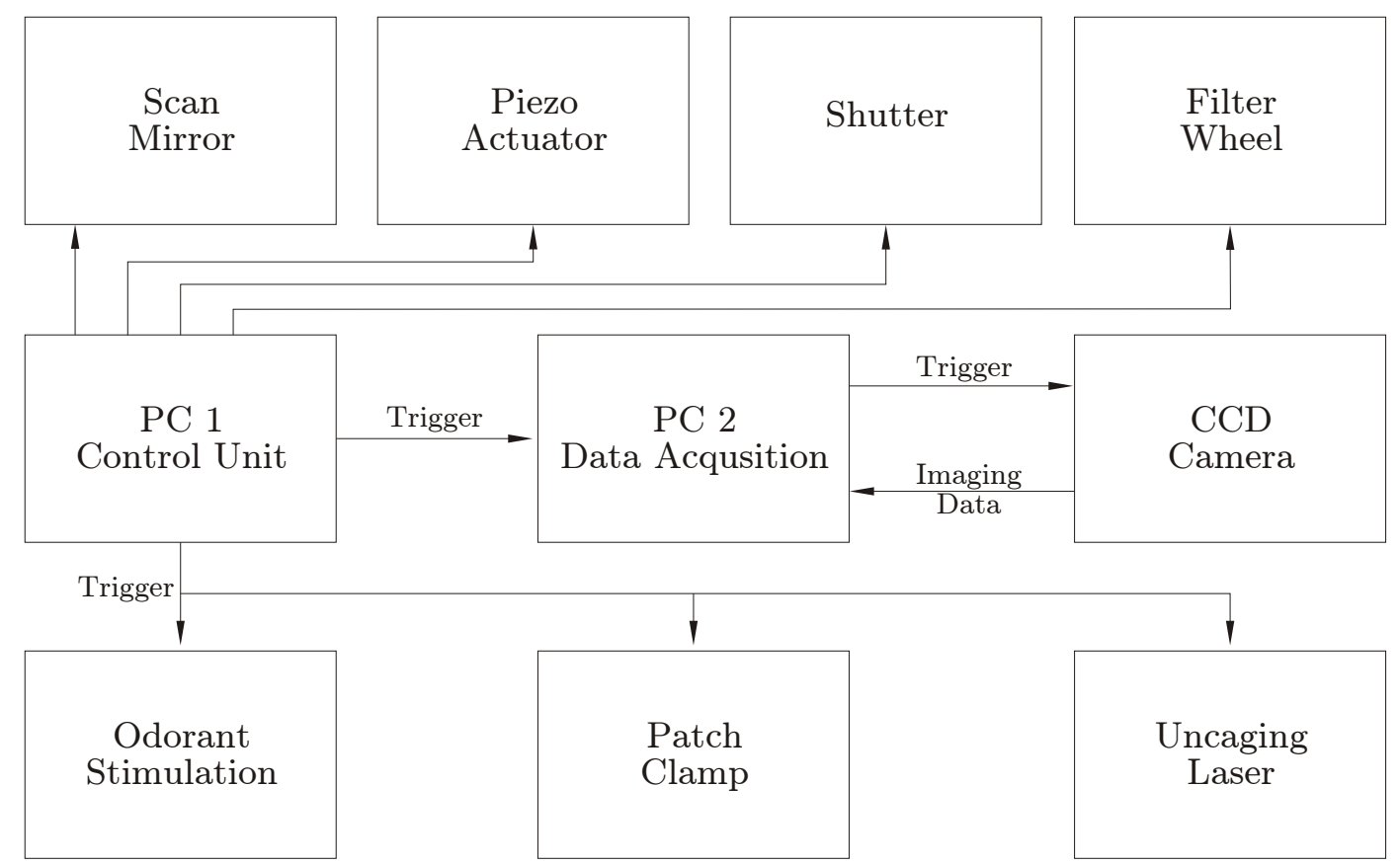

Figure 2.1: Overview of the control system of the scanning microscope. The 'master control' of the setup is $\mathrm{PC} 1$ that produces the signals for all electronic components (scan mirror, piezo actuator, shutter, camera trigger, external trigger). The camera trigger is fed into a $\mathrm{PC} 2$ which is used for the image acquisition. The external trigger can be used to synchronize the data acquisition to other elements of the experimental setup, such as the odor application system or a patch clamp unit.

resolution during a single acquisition, as used in the Results (see Fig. 3.15).

\subsubsection{Data acquisition system}

The data acquisition trigger supplied by the master PC is fed into the PC used for image acquisition. The software omap custom written in $\mathrm{C}++$ by Mihai Alevra provides the interface to set image acquisition parameters and it controls the actual image acquisition by communicating with the CCD camera. The image data of each acquisition, which can be up to 4-dimensional, are directly written to the hard drive into a single file. These data can be visualized and analyzed using software custom written for MATLAB by Stephan Junek. 
a Scan Mirror Position ( $y$-position)

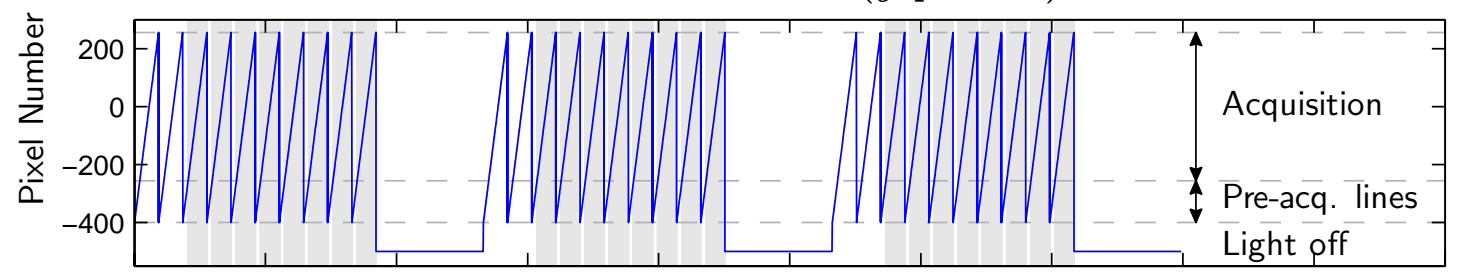

b

Piezo Actuator ( $z$-position)

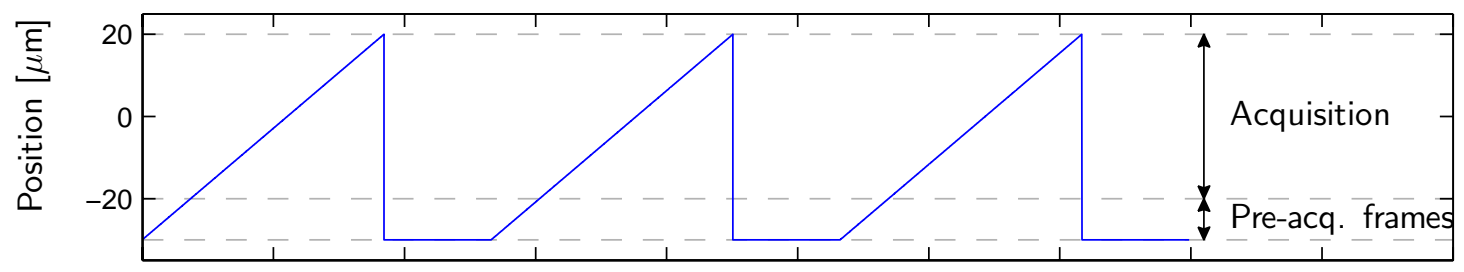

C

Output Trigger

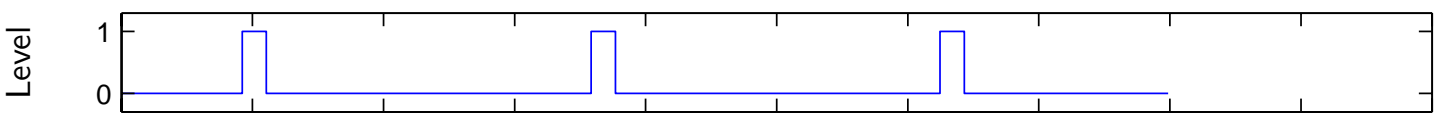

d

Shutter Trigger

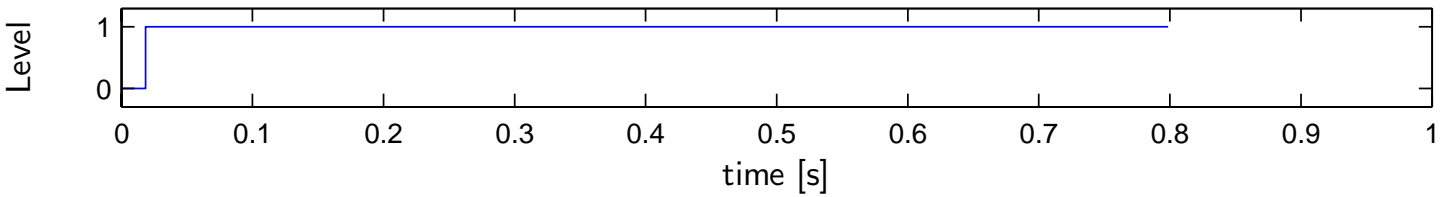

Figure 2.2: Schematics of a scan protocol used to control the electronic components of the microscope. (a) The scan mirror is controlled by a periodic stair-case like function. The image acqusition is paused during the 'pre-acquisition lines' to exclude the return movement of the scan mirror from the acquisition. When deflecting the scan mirror strongly, the light does not reach the objective and the sample is not illuminated ('Light-off'). This can be used as a fast shutter to introduce short breaks in the acquisition. The image acquisition periods are indicated by gray bars. (b) The piezo actuator is driven by a ramp function. Similarly to the scan mirror, the return of the objective can be excluded from the acquisition interval by the definition of 'pre-acquisition frames'. (c) The output trigger can be used to control external devices, such as the odor application system. (d) Another trigger is used to control the shutter, thereby restricting the illumination of the sample to the time of the experiment. 


\subsubsection{Tuning and characterization of scan mirror and piezo actuator}

The behavior of the scan mirror and the piezo actuator were evaluated using custom written routines (MATLAB, The MATHWorks, USA) to generate command patterns and read out the monitor signal from the control boards. Signals were sampled at $25 \mathrm{kHz}$. This configuration was used to adjust the settings of the proportional - integral - differential controllers to achieve optimal performance for the experimentally relevant parameters. In case of the piezo actuator, the dependence of the response on the weight of the objective motivated the addition of a second control board. The user can now select from two settings, one optimized for light and fast responding objectives, the other one optimized for heavy and inert objectives. For display purposes, the signals were filtered off-line at $10 \mathrm{kHz}$.

\subsection{Slice Preparation and Physiological Experiments}

\subsubsection{Slice preparation}

Tadpoles of Xenopus laevis (stage 51 - 54; staged after Nieuwkoop and Faber (1967)) were chilled in a mixture of ice and water and decapitated, as approved by the Göttingen University Committee for Ethics in Animal Experimentation. A block of tissue containing the olfactory mucosa, the olfactory nerves and the anterior two-thirds of the brain were cut out and kept in bath solution containing (in $\mathrm{mM}$ ): $98 \mathrm{NaCl}$,

$2 \mathrm{KCl}, 1 \mathrm{CaCl}_{2}, 2 \mathrm{MgCl}_{2}, 5$ glucose, 5 sodium pyruvate, 10 Hepes; 230 mosmol, pH 7.8. The tissue block was glued onto the stage of a vibroslicer (VT 1000S, Leica, Bensheim, Germany) and cut horizontally into approximately $200 \mu \mathrm{M}$-thick slices (Manzini et al., 2002). The slices were transfered into custom built microscopy chambers and stabilized with a grid (Edwards et al., 1989).

\subsubsection{Staining protocols}

\section{Bath incubation}

For the staining solution, Fluo-4/AM (Molecular Probes, Karlsruhe, Germany) was first dissolved in 20\% Pluronic F-127 in DMSO, and then diluted in the bath 
solution to reach the final concentration. To avoid transporter-mediated destaining of the slices, MK571 (50 $\mu \mathrm{M}$; Alexis Biochemicals, Lörrach, Germany), a specific inhibitor of multidrug resistance-associated proteins, was added to the staining solution (Manzini and Schild, 2003b; Manzini et al., 2008). For 2D imaging, the tissue slices were incubated in a solution with dye concentration of $2-5 \mu \mathrm{M}$ for $30 \mathrm{~min}$, followed by a post-incubation period of 30 min in ringer solution with MK571 (50 $\mu \mathrm{M})$.

\section{Bolus loading}

For 3D imaging, a solution containing $100-500 \mu \mathrm{M}$ Fluo-4/AM was pressureinjected at a depth of approx. $70 \mu \mathrm{m}$ using patch pipettes $(6-8 \mathrm{M} \Omega, 50-100 \mathrm{hPa}$ for $1-5 \mathrm{~min}$ ) into two sites per bulb hemisphere (adopted from Garaschuk et al. (2006); Stosiek et al. (2003)). The progress of the injection was monitored using a $40 \times$ water immersion objective. The spontaneous activity of the neurons was investigated after an incubation period of $30-40$ min following the last injection.

\section{Staining of ORNs by electroporation}

The animal was anesthetized in ice cold water, placed on a preparation dish covered with silicon, and gently fixed by restraining its movements with fine needles. The caudal part of the tadpole was covered with wet cellulose tissue. Small crystals (1$10 \mu \mathrm{g}$ ) of Fluo-4 dextran (Invitrogen, Karlsruhe, Germany) were placed in both nasal cavities. After the crystals dissolved, two platinum electrodes were inserted in the nasal cavities. The dye was transferred into the cells by electroporation. Six pulses of $20 \mathrm{~V}$ and $20 \mathrm{~ms}$ duration were applied, with a break of $5 \mathrm{~min}$ between the third and fourth pulse. After another $5 \mathrm{~min}$, the animal was placed back into the water. Experiments were carried out $1-2$ days later. This staining procedure was established by Eugen Kludt, Department of Neurophysiology and Cellular Biophysics, University Göttingen. 


\subsubsection{Application of odorants}

\section{Application system}

For odor application experiments, the recording chamber was constantly perfused by gravity feed from a syringe through a slightly modified version of a funnel applicator described earlier (Manzini, 2002; Schild, 1985). The tip of the applicator was placed in front of the ipsilateral mucosa using a $10 \times$ objective. The odorants were applied into the funnel without stopping the flow of the bath solution using an electronic pipette (HandyStep electronic, BRAND, Wertheim, Germany). The pipette was modified to control the outflow with the trigger signal (TTL) provided by the control PC. The timing and reproducibility of the stimulus application was measured by adding fluorescent dye ( $1 \mu \mathrm{M}$ Fluorescein, Sigma, Deisenhofen, Germany) to the stimulus solution, and imaging the outflow from the tip of the applicator with a $10 \times$ objective at $400 \mathrm{~Hz}$. The delay was measured to be $(363 \pm 9) \mathrm{ms},(436 \pm 19) \mathrm{ms}$ and $(716 \pm 108) \mathrm{ms}$ for $10 \%, 50 \%$ and $90 \%$ of the maximum concentration, respectively (see Fig. 3.15b). The absolute time of this delay might slightly vary from slice to slice due to the positioning of the applicator relative to the mucosa. Fluid was removed from the recording chamber using a syringe needle connected to a vacuum pump (Hyflo Model C vacuum pump, Medcalf Brothers Ltd., Potters Bar, England) via a waste-bottle.

\section{Odor stimuli}

The olfactory system was stimulated with solutions containing amino acids or an extract of amphibia food based on Spirulina algae (Mikrozell, Dohse Aquaristik, Bonn, Germany). The amino acids (Sigma, Deisenhofen, Germany) were dissolved in bath solution (10 $\mathrm{mM}$ stock) and diluted prior to the experiment to the final concentrations as indicated in the text. The amino acids were applied individually or as a mixture of 15 amino acids (L-proline, L-valine, L-leucine, L-isoleucine, L-methionine, L-glycine, L-alanine, L-serine, L-threonine, L-cysteine, L-arginine, L-lysine, L-histdine, L-tryptophane, L-phenylalanine), excluding amino acids that could have a direct effect on the neurons in the OB (L-glutamate, L-aspartate, L- 
glutamine and L-asparagine). For the solution containing the food extract, $0.5 \mathrm{~g}$ of Spirulina algae were dissolved in $100 \mathrm{ml}$ bath solution and filtered through a single use filter $(0.5 \mu \mathrm{m}$ pore size, Minisart, Sartorius AG, Göttingen, Germany). Application of bath solution was used as a negative control. Odors were applied in a randomized order, with a minimum interstimulus duration of $1.5 \mathrm{~min}$.

\subsubsection{Microscopy}

The custom built line-illumination microscope, described in detail in 3.1, was used for all image acquisition except for imaging the results of the dye injection experiments in 3.2. After dye injection, slices were imaged using an Axiovert 100M equipped with a laser-scanning unit LSM 510 (ZEISS, Jena, Germany). Alexa Biocytin 532 was imaged in the red channel (helium/neon laser, $543 \mathrm{~nm}$, NFT 545, LP 560). The green channel (Fluo-4) was imaged for alignment with the physiological recordings from the line illumination microscope (argon ion laser, $488 \mathrm{~nm}$, HFT 488/53, BP 505-550). The following objectives were used as indicated in the text: $25 \times$ LD LCI Plan-Apochromat $0.8 \mathrm{~W} ; 40 \times$ Achroplan $0.8 \mathrm{~W} ; 63 \times$ Achroplan $0.95 \mathrm{~W}$; 40× C-Apochromat $1.2 \mathrm{~W} ; 10 \times$ Plan-NeoFluar 0.3 (all ZEISS, Jena, Germany). For each experiment, 'dark images' were acquired by closing the laser shutter with otherwise identical acquisition parameters. These images were used for background estimation.

\subsubsection{Electrophysiology}

Patch clamp was performed using an EPC7 plus amplifier (HEKA, Germany) and pipettes with a series resistance between 8 and $12 \mathrm{M} \Omega$. Alexa Biocytin 532 (Invitrogen, Germany) was added to the intracellular solution (in mM: $2 \mathrm{NaCl}$, $11 \mathrm{KCl}, 2 \mathrm{MgSO}_{4}, 80 \mathrm{~K}$-Gluconat, 10 Hepes, 0.2 EGTA, $2 \mathrm{Na}_{2}$ ATP, $0.1 \mathrm{Na}_{2} \mathrm{GTP}$ ). After breaking the seal, the cell was held at $-65 \mathrm{mV}$ for one minute to allow diffusion of the dye into the cytosol. 


\subsection{Data Analysis}

All data analysis was performed using custom software written in MATLAB (THE MATHWORKS, USA).

\subsubsection{Characterization of point spread functions}

In order to determine the width of the point spread functions (PSF) of the microscope, image stacks of individual fluorescent silica beads with a diameter of $175 \mathrm{~nm}$ (Fluospheres, Molecular Probes, Karlsruhe, Germany) were analyzed and the resulting values averaged. The analysis consisted of three steps:

1. Determination of the 'center of gravity' of the intensity distribution. The voxel closest to this position was used as the center of the PSF. Using neighbouring voxels or interpolating the intensities into the sub-pixel position of the bead center did not significantly alter the following results.

2. The $(x, y)$-image plane through the bead center was used to determine the lateral size of the PSF. The intensity distribution in the $(x, y)$-plane was fitted with a two-dimensional Gaussian.

3. Similarly, the axial intensity distribution along the center of the bead was determined, and fitted with a one-dimensional Gaussian.

From the fit values in the three spatial directions, the full width at half maximum (FWHM) was calculated for each bead, and the mean and standard deviation of these values were calculated from many beads for each objective.

\subsubsection{Image analysis}

Drift of the slice was corrected whenever necessary using a custom written routine. The image data recorded by the CCD camera were transformed into $\Delta F / F_{0}=$ $\left(F(t)-F_{0}\right) / F_{0}$ values after subtraction of the background. $F_{0}$ was determined as the mean of the intensity values of the first 10 data points for each pixel. 


\subsubsection{Autocorrelation map}

To facilitate the identification of neurons that respond to a given stimulus reproducibly, an 'autocorrelation map' was calculated. This map is calculated on a pixelby-pixel basis. For each pixel, the time traces $I_{a}(t)(t=1, \ldots, T)$ for all applications $a \in\{1, \ldots, A\}$ of a given stimulus were concatenated into a single vector $I^{*}(t)$ $(t=1, \ldots, A \cdot T)$. For this vector, autocorrelation values $C$ were calculated for time shifts that are multiples of one application sequence $T$ :

$$
C(n)=\frac{\sum_{t=1}^{A T}\left(I^{*}(t+n T \bmod A T)-\overline{I^{*}}\right) \cdot\left(I^{*}(t)-\overline{I^{*}}\right)}{\sum_{t=1}^{A T}\left(I^{*}-\overline{I^{*}}\right)^{2}},
$$

for $n=1, \ldots,(A-1)$. The values $C(n)$ of each pixel were averaged. The result is map that indicates positions which exhibited similar time courses during the repeated applications of the stimulus. As the time courses of $\mathrm{Ca}^{2+}$ indicators show transients with decay times around $1 \mathrm{~s}$ due to the delayed removal of $\mathrm{Ca}^{2+}$ from the cytosol, this map is insensitive to small temporal fluctuations in the activity patterns. This map was used as a guide for placing the regions of interest (ROIs). This procedure was additionally guided by visually evaluating the fluorescent time courses from all applications.

\subsubsection{Activity correlation imaging}

Drift of the slice was corrected whenever necessary using a custom written routine. A bleach correction was performed for all pixels by subtracting a linear trend from each pixel's time trace. The reference traces were obtained by averaging the fluorescence intensities across individual regions of interests (ROIs) for each time point. To facilitate the selection of regions of interest, a 'pixel correlation map' was obtained by calculating the cross-correlation between the fluorescence signals of a pixel to that of its immediate neighbors and then displaying the resulting value as a grayscale map. As physiological responses often give similar signals in adjacent pixels, this method specifically highlights those pixels. In contrast, pixels that contain only noise show uncorrelated traces and thus appear dark in the cross-correlation map (Manzini et al., 2007a). The ROIs were then selected semi-automatically, based on the correlation between the time traces of a manually selected pixel and the time 
traces of the pixels in a certain neighborhood (approx. $20 \times 20 \times 20 \mu^{3}$ ) around this pixel. The correlation map for the $j^{\text {th }}$ ROI was then created by calculating the correlation coefficients between the reference trace $r_{j}(t)$ and the time traces $v_{i}(t)$ of each pixel. The $i^{\text {th }}$ pixel in the $j^{\text {th }}$ correlation map gets thus assigned the value

$$
c_{i j}=\frac{\sum_{t=1}^{T}\left(v_{i}(t)-\overline{v_{i}}\right)\left(r_{j}(t)-\overline{r_{j}}\right)}{\left\|v_{i}(t)-\overline{v_{i}}\right\| \cdot\left\|r_{j}(t)-\overline{r_{j}}\right\|},
$$

with $T$ being the number of time points of the observation, and $\|\cdot\|$ denoting the 2-norm of the respective vector. Displayed correlation maps were in some cases mildly Gauss-filtered (width $<1$ pixel, indicated in the text). 


\section{3}

\section{RESULTS}

\subsection{Design, Realization and Characterization of a Fast Con- focal Line-Scanning Microscope}

\subsubsection{Design of the optical pathway}

A confocal laser scanning microscope that projects a line of excitation light (instead of a point) onto the sample was designed and built (Fig. 3.1). The object is scanned in one direction only, perpendicular to the excitation line. Provided a fast sensor and acquisition system, high frame rates can thus be achieved. This microscope will be referred to as Line Illumination Microscope (LIM).

\section{Excitation pathway}

In a conventional laser scanning microscope, the arrangement of the optical elements (scan mirrors, scan lens, tube lens and objective; Pawley (2006)) results in a focus of the laser beam in the front focal plane of the objective (Fig. 3.2, bottom panel). In order to create an illumination line that is scanned across the sample, this optical design is modified by adding a cylindrical lens in front of the dichroic mirror (Fig. 3.2, top panel). In this way a focus of the initially parallel laser beam is created in only one direction on the scan mirror, and subsequently in the back-focal plane of the objective. Due to the introduced anisotropy in the light beam, the light propagation has to be discussed separately for the two lateral directions (i.e. directions perpendicular to the optical axis). The direction of the focus line on the scan mirror will be referred to as the $x$-direction, the $y$-direction being perpendicular to it. Figure 3.2 shows the light propagation of the excitatory pathway for both 


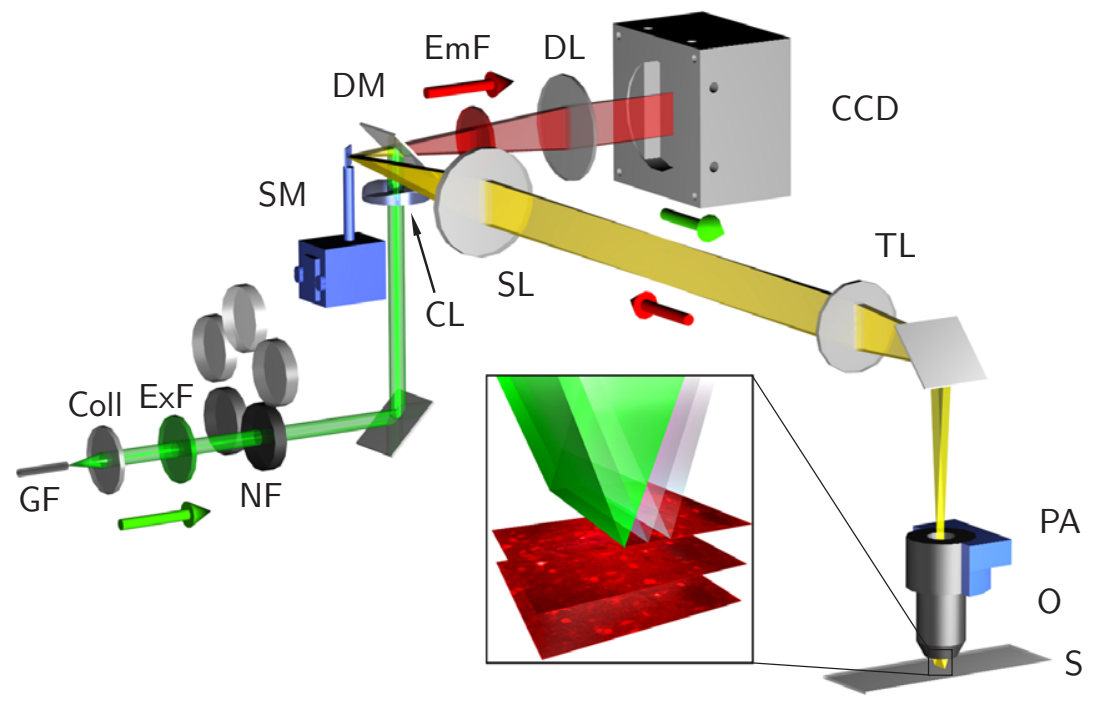

Figure 3.1: Schematic of the complete assembly of the line illumination microscope. The foci of the beam can be recognized, arrows indicate the direction of light propagation (green: excitation light, red: emission light, yellow: 'overlap' of excitation and emission light). The inset illustrates the scan scheme for the acquisition of an image stack: The light is focussed into a line in the object plane, which is scanned across the sample for the acquisition of a single frame. Changing the position of the objective with the piezo actuator allows to move the imaged plane along the optical axis.

Abbreviations GF: glass fiber, Coll: collimator, ExF: excitation filter, NF: neutral density filter, CL: cylindrical lens, DM: dichroic mirror, SM: scan mirror, SL: scan lens, TL: tube lens, PA: piezo actuator, Obj: objective, S: specimen (object plane), EmF: emission filter, DL: detector lens, CCD: CCD camera.

directions (the scan mirror position is indicated, while the deflection caused by the mirror is omitted for clarity). The introduction of the cylindrical lens results in a parallel light beam in the $x$-direction in front of the objective. Since the $y$-direction is not affected by the cylindrical lens, the objective focuses the light into the object plane along the $y$-direction, and the desired 'focussed line' is created. One of the two scan mirrors used in conventional confocal microscopes becomes thus obsolete. While the light propagation in $y$-direction is the same as in a confocal microscope, the $x$-direction resembles the pathway of a widefield microscope. 


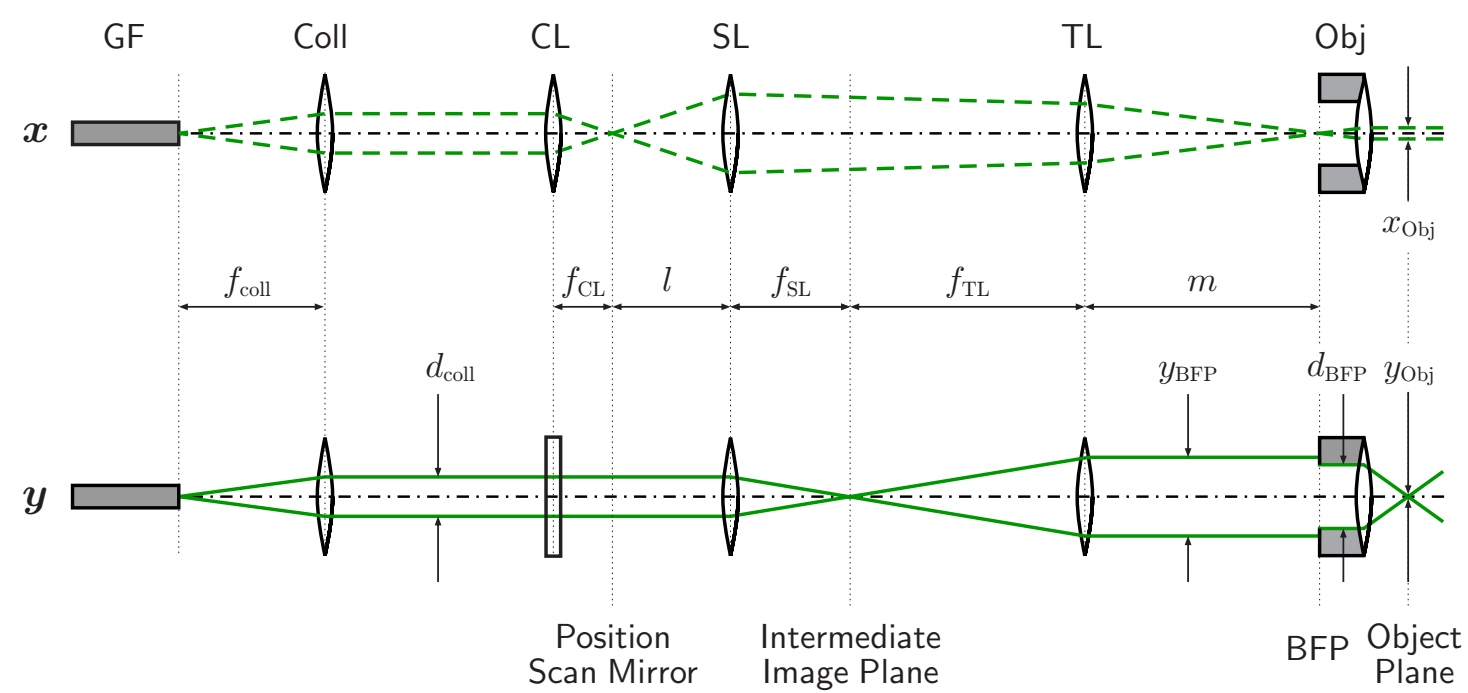

Figure 3.2: The optical arrangement for the excitation pathway of the LIM in $x$-direction (top, broken lines) and $y$-direction (bottom, solid lines) direction. The two directions differ due to the cylindral lens that introduces a focus in $x$-direction on the scan mirror and in the objective's backfocal plane. The light consequently propagates through the object plane in a parallel and focussed fashion for the $x$ - and $y$-directions, respectively. In this way, a focussed line is created in the object plane. While the arrangement of elements for the $y$-direction is identical to a conventional confocal point-scanning microscope, the optical pathway of the $x$-direction resembles a widefield microscope. For the sake of clarity, only the lenses, but no filters and mirrors are shown. The indicated variables refer to the calculations of the pathway in the text.

Abbreviations GF: glass fiber, Coll: collimator, CL: cylindrical lens, SL: scan lens, TL: tube lens, BFP: backfocal plane of the objective, Obj: objective.

In order to assure good imaging properties, two requirements have to be satisfied: (1) The length $x_{\mathrm{Obj}}$ of the line should be significantly larger than the desired field of view, since the Gaussian profile of the laser beam will lead to a drop of intensity toward the periphery of the line. (2) The width $y_{\mathrm{Obj}}$ of the line in the object plane (i.e. the focus width) should be very small to achieve good spatial resolution.

The width of the focus $y_{\mathrm{Obj}}$ depends on the excitation wavelength $\lambda_{\text {exc }}$, the numerical aperture $N A$ of the objective and the ratio of the beam diameter in the back-focal plane $y_{\mathrm{BFP}}$ and the diameter of the back-focal aperture $d_{\mathrm{BFP}}$ (Fig. 3.2). 
The width of the line can be calculated according to (Wilhelm et al., 2003)

$$
y_{\mathrm{Obj}}=0.71 \cdot \frac{\lambda_{\mathrm{exc}}}{N A} \cdot \sqrt{0.51+\frac{0.28}{\left(y_{\mathrm{BFP}} / d_{\mathrm{BFP}}\right)^{2}}},
$$

measured as full width at half maximum (FWHM). Expressing this value as a multiple of airy units $\left(1 \mathrm{AU}=1.22 \cdot \frac{\lambda_{\mathrm{exc}}}{N A}\right)$ removes the explicit dependency on wavelength and $N A$ :

$$
y_{\mathrm{Obj}}=\frac{0.71}{1.22} \cdot \mathrm{AU} \cdot \sqrt{0.51+\frac{0.28}{\left(y_{\mathrm{BFP}} / d_{\mathrm{BFP}}\right)^{2}}} .
$$

Equation 3.1 shows that there is a fundamental lower bound for the width of the focus given by $y_{\mathrm{Obj}} \geq 0.42 \cdot \mathrm{AU}\left(\right.$ as $\left.y_{\mathrm{BFP}} / d_{\mathrm{BFP}} \rightarrow \infty\right)$. This limit is approached for large beam diameters in the back-focal plane. A focus width of $y_{\mathrm{Obj}} \leq 0.5 \cdot \mathrm{AU}$ thus requires $y_{\mathrm{Obj}} \geq 1.1 \cdot d_{\mathrm{BFP}}$, where $y_{\mathrm{BFP}}$ depends on the diameter of the collimated laser beam $d_{\text {coll }}$ and the focal length of scan and tube lens (Fig. 3.2)

$$
y_{\mathrm{BFP}}=d_{\mathrm{coll}} \cdot \frac{f_{\mathrm{TL}}}{f_{\mathrm{SL}}} .
$$

The length of the line $x_{\mathrm{Ob} j}$ can be calculated by means of geometrical optics from the initial beam diameter $d_{\text {coll }}$ and the focal length of cylindrical lens, scan lens and the magnification $M$ of objective and tube lens (Fig. 3.2):

$$
x_{\mathrm{Obj}}=d_{\mathrm{coll}} \cdot \frac{f_{\mathrm{SL}}}{f_{\mathrm{CL}}} \cdot \frac{1}{M}
$$

The field of view is chosen to enable observation of a complete Xenopus laevis tadpole olfactory bulb with a $25 \times$ objective, i.e. to be in the range of $400 \mu \mathrm{m} \times 400 \mu \mathrm{m}$ for this magnification.

The requirements for both $x_{\mathrm{Obj}}$ and $y_{\mathrm{Obj}}$ can be satisfied by expanding the laser beam to a sufficiently large diameter $d_{\text {coll }}$. The following values were chosen for the focal length of the various lenses: $f_{\mathrm{CL}}=40 \mathrm{~mm}$ for the cylindrical lens (LinOs, Göttingen, Germany), $f_{\mathrm{SL}}=80 \mathrm{~mm}$ for the scan lens (Linos, Göttingen, Germany) and $f_{\mathrm{TL}}=$ $164.5 \mathrm{~mm}$ for the tube lens (ZEISs, Göttingen, Germany). The diameter $d_{\mathrm{BFP}}$ of the objective backfocal plane is different for each objective $(10.6 \mathrm{~mm}, 6.6 \mathrm{~mm}$ and $5.0 \mathrm{~mm}$ for the $25 \times, 40 \times$ and $63 \times$ objectives listed below, respectively). Choosing the diameter of the collimated beam $d_{\text {coll }}=12 \mathrm{~mm}$ results in the values for $x_{\text {Obj }}$ and $y_{\text {Obj }}$ listed in Table 3.1. For these three objectives the width of the line is close to 
Table 3.1: Size of the illumination line and pixel scale for three different objectives as specified in the Materials and Methods. The values for the length $x_{\mathrm{Obj}}$ and the width $y_{\mathrm{Obj}}$ of the line as well as the pixel scale as multiples of airy units are derived from the calculations in the text, while the pixel scale in $\mu \mathrm{m}$ and the size of the field of view result from measuring a standardized grid.

\begin{tabular}{ccccccc}
\hline Objective & $\begin{array}{c}x_{\mathrm{Obj}} \\
{[\mu \mathrm{m}]}\end{array}$ & $\begin{array}{c}y_{\mathrm{Obj}} \\
{[\mu \mathrm{m}]}\end{array}$ & $\begin{array}{c}y_{\mathrm{Obj}} \\
{[\mathrm{AU}]}\end{array}$ & $\begin{array}{c}\text { Pixel Scale } \\
{[\mathrm{AU}]}\end{array}$ & $\begin{array}{c}\text { Pixel Scale } \\
{[\mu \mathrm{m}]}\end{array}$ & $\begin{array}{c}\text { Field of View } \\
{[\mu \mathrm{m} \times \mu \mathrm{m}]}\end{array}$ \\
\hline $25 \times 0.80 \mathrm{~W}$ & 960 & 0.32 & 0.44 & 1.18 & 0.90 & $461 \times 461$ \\
$40 \times 0.80 \mathrm{~W}$ & 600 & 0.32 & 0.42 & 0.73 & 0.54 & $276 \times 276$ \\
$63 \times 0.95 \mathrm{~W}$ & 380 & 0.26 & 0.42 & 0.55 & 0.30 & $154 \times 154$ \\
\hline
\end{tabular}

the theoretical limit of $0.42 \mathrm{AU}$ and the length of the line is about twice as large as the field of view. The selected configuration therefore meets the aforementioned demands. The beam diameter of $d_{\text {coll }}=12 \mathrm{~mm}$ is achieved by using a lens with focal length $f_{\text {coll }}=d_{\text {coll }} /\left[2 \cdot \tan \left(\arcsin N A_{\mathrm{GF}}\right)\right]=50 \mathrm{~mm}($ Linos, Göttingen, Germany) to collimate the laser beam emerging from the single mode glass fiber with numerical aperture $N A_{\mathrm{GF}}=0.12$ (LAser Components, Olching, Germany). The position of the scan mirror (GSI Luomincs, Billerica, USA), which has to be placed in a conjugated plane to the back-focal plane of the objective, is determined through the (fixed) distance $m=152 \mathrm{~mm}$ between the tube lens and the back-focal plane of the objective (Fig. 3.2, (Gennerich, 1999)):

$$
l=f_{\mathrm{SL}}\left[1+\frac{f_{\mathrm{SL}}\left(f_{\mathrm{TL}}-m\right)}{f_{\mathrm{TL}}^{2}}\right]=82.9 \mathrm{~mm} .
$$

The complete design of the microscopic setup including both excitation (green lines) and emission (red lines) pathways, as well as all optical elements (excitation filter: z488/10, emission filter: HQ532/70, dichroic mirror: z488rdc, all from AHF ANALYSENTECHNIK, Tübingen, Germany) is depicted schematically in Figure 3.3 and in the final 3D assembly in Figure 3.1. An argon ion laser (LASER TECHNOLOGIES, Kleinostheim, Germany) at $488 \mathrm{~nm}$ is used as light source. The maximum laser intensity of $250 \mathrm{~mW}$ ensures sufficient illumination intensity across the line. 

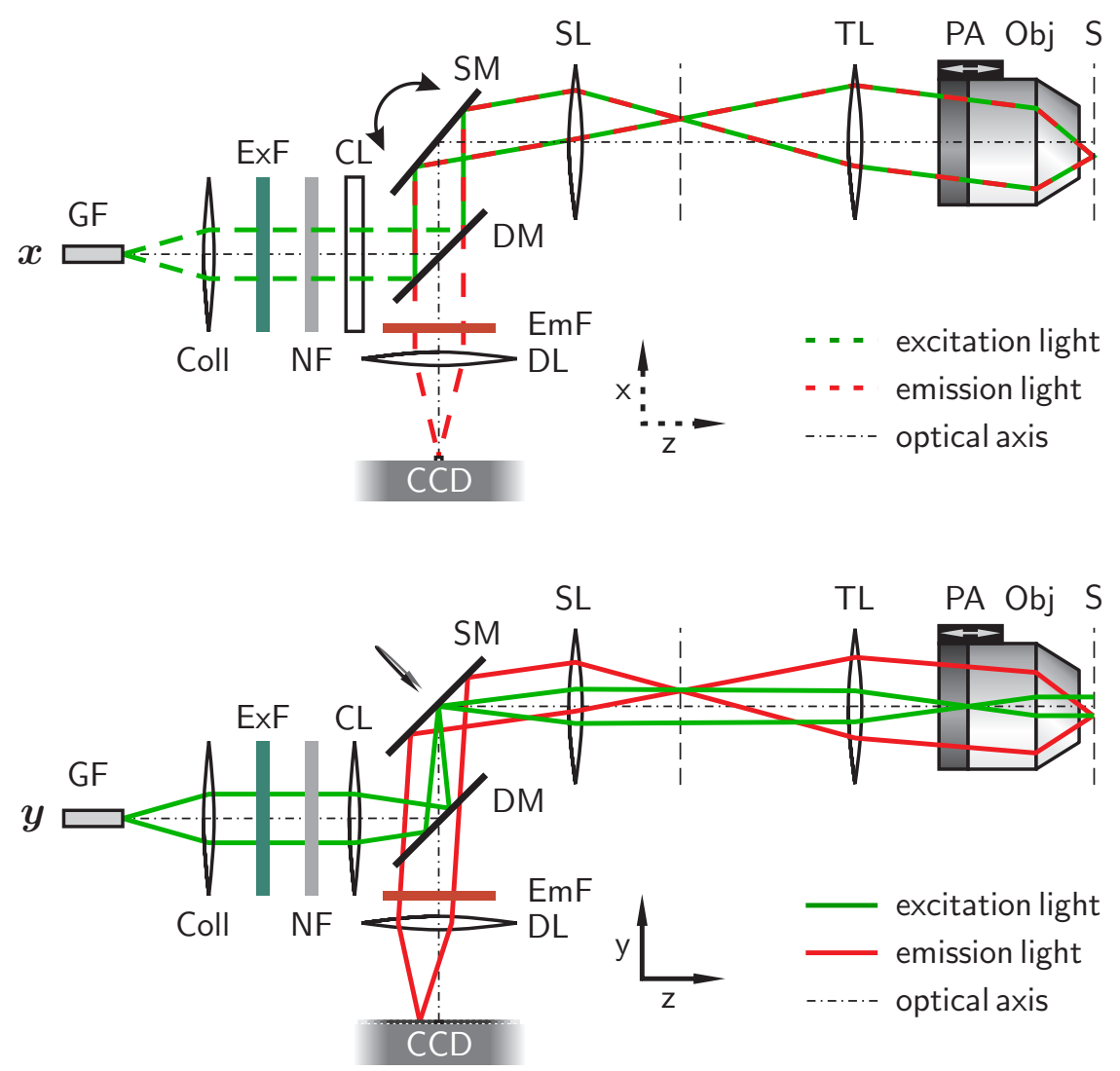

Figure 3.3: Propagation of beams for excitation and emission pathways. Schematic of the propagation of the light rays for the $x$-direction (top, broken lines) and the $y$-direction (bottom, solid lines) for both excitation (green) and emission (red) light. By inclining the scan mirror, the line is scanned across the sample. In $x$-direction, the mirror de-scans the emission light, while in $y$ direction the line is imaged onto the linear CCD array at any time. Behind the dichroic mirror, a single lens forms the image on the sensor. The width of the CCD pixel acts as the confocal aperture.

Abbreviations GF: glass fiber, Coll: collimator, ExF: excitation filter, NF: neutral density filter, CL: cylindrical lens, DM: dichroic mirror, SM: scan mirror, SL: scan lens, TL: tube lens, PA: piezo actuator, Obj: objective, S: specimen (object plane), EmF: emission filter, DL: detector lens, CCD: CCD camera.

\section{Emission pathway}

In $x$-direction, each point of the line is imaged onto a different position on the detector, while in $y$-direction the tilt of the scan mirror ensures that the deflection of the 
focus is compensated and that the line is always projected onto the same $y$-position on the detector ('de-scanned'). Similarly to the excitation pathway, the $y$-direction resembles a conventional confocal scanning microscope, while the $x$-direction is reminiscent of a widefield microscope.

High-speed 2D or time-lapsed 3D imaging usually requires the acquisition of a large number of images, which is typically accompanied by significant bleaching of the fluorescent dye. The design of the emission pathway was thus optimized for maximum efficiency by reducing the number of optical elements. To this end, advantage was taken of the properties of the detector, a fast read-out, linear CCD array consisting of 512 pixel (AViiVA SM2 CL0514, Atmel, France). The small size of the pixel $(14 \mu \mathrm{m} \times 14 \mu \mathrm{m})$ allows to use the pixel width as the confocal aperture. While this design sacrifices the ability to adjust the thickness of the optical section, it maximizes photon efficiency by omitting the associated optics required for a confocal aperture. Consequently, a single lens behind the dichroic mirror suffices to focus the emission light onto the CCD camera (Fig. 3.3). The fact that the CCD array acts as both detector and the confocal aperture, puts consequently a constraint on the magnification from the object plane to the plane of the sensor: most of the light emanating from the (finite-sized) focus in the object plane (Eq. 3.1) has to be projected onto the CCD-pixel, while the out-of-focus light should not reach the detector. The total magnification from the object plane to the position of the sensor $M_{\text {tot }}$ is the product of the magnification of the objective / tube lens $\left(M_{\mathrm{Obj}}\right)$ and the magnification of the telescope-like pair scan lens / detector lens $\left(M_{\mathrm{SL} / \mathrm{DL}}=f_{\mathrm{DL}} / f_{\mathrm{SL}}\right)$ : $M_{\mathrm{tot}}=M_{\mathrm{Obj}} \cdot M_{\mathrm{SL} / \mathrm{DL}}$. The width of the line at the sensor is thus

$$
y_{\mathrm{CCD}}=M_{\mathrm{tot}} \cdot y_{\mathrm{Obj}}=0.5 \mathrm{AU} \cdot M_{\mathrm{tot}}=0.5 \mathrm{AU} \cdot M_{\mathrm{SL} / \mathrm{DL}} \cdot M_{\mathrm{Obj}}
$$

The width of the image of the line depends on the magnification of the objective, resulting in a dependency of the optical sectioning properties on the choice of objective. For a conventional LSM, a pinhole size of $1 \mathrm{AU}$ is a good compromise between signal strength and resolution (Pawley, 2006). The magnification of the lens pair scan lens / detector lens was thus chosen to achieve a pixel scale (i.e. width of the confocal aperture) of approximately 1 AU for the $25 \times$ objective by selecting a focal length of $f_{\mathrm{DL}}=50 \mathrm{~mm}$ for the detector lens (Linos, Göttingen, Germany). The 
pixel scale for the higher magnification objectives is consequently smaller than $1 \mathrm{AU}$ (Tab. 3.1). To determine the precise magnification from the object plane into the detector plane for different objectives, images of standardized gold grids (PLANO, Wetzlar, Germany) were taken and analyzed. The pixel scales measured from these images and the respective dimensions of the field of view (for $512 \times 512$ square pixel) are provided in Table 3.1. It should be mentioned that the pixel width in $y$-direction can be adjusted by changing the amplitude of the scan mirror movements. This can be used to either increase the sampling density (smaller scan steps) or to increase the field of view (larger scan steps). For most experiments, including the investigation of the spatial resolution in 3.1.2, the sampling in $y$-direction was chosen to be identical to the fixed pixel width in $x$-direction.

\section{The axial position of the object plane}

The object is mounted on a piezo - driven actuator (PI, Karlsruhe, Germany) that allows the adjustment of the $z$-position of the object plane (Fig. 3.3). The change of the position of the objective's back-focal plane relative to the excitation beam is small $(\leq 100 \mu \mathrm{m})$ compared to the focal length of the tube lens $\left(f_{\mathrm{TL}}=164.5 \mathrm{~mm}\right)$. It does thus not have a significant effect on the properties of the illumination line.

\section{Characterization of the microscopic setup}

Before using a novel tool to address biological questions, it is necessary to characterize and evaluate its performance. This analysis provides the technical range of potential applications and the expertise to select the optimal settings for a given experiment. The LIM was designed to enable functional imaging with high temporal resolution in $2 \mathrm{D}$ and $3 \mathrm{D}$. This aim puts demands on the technical abilities of the setup (high acquisition rates have to be realized) as well as on the biological specimen under observation (a large number of frames has to be acquired). Especially the limitations posed by the sensitivity of biological specimen will often require a tradeoff between experimental parameters (e.g. temporal vs. spatial resolution), making a good understanding of the technical possibilities even more crucial. Specifically the following factors are important for the investigations of neuronal network dynamics: 
- To decide which structures of a given network can be resolved (olfactory glomeruli $>$ somata $>$ dendrites $>$ axons), the spatial resolution in $x$-,y- and $z$-direction has to be known.

- To decide which kind of temporal processes can be studied, the temporal resolution for both 2D- and 3D-imaging needs to be known.

\subsubsection{Spatial resolution}

The cylindrical lens in the excitation pathway and the slit-like confocal aperture of the CCD sensor introduce two principle axes in the image plane. Apart from the anisotropy of the beam and the aperture, these two directions differ in that the sampling density is fixed along the $x$-direction (for a given objective) by the pixel pitch of the CCD sensor, while it can be adjusted in the $y$-direction by changing the amplitude of the scan mirror movement. The resolution of the microscope was investigated for isotropic sampling intervals along the $x$ - and $y$-directions.

\section{Resolution limits in optical microscopy}

The resolution of an imaging system is defined as the minimum distance at which two objects can be distinguished. For conventional imaging systems it is fundamentally limited by the wave nature of light, restricting the resolution to the range of the wavelength of the light (Abbe (1873), but see Betzig et al. (2006) and Hell (2003) for recently developed exceptions). The second important factor for the resolution of an imaging system is the numerical aperture $(N A)$ of the objective, since it determines how strongly the light is focussed and which spatial frequencies of the light emanating from the object are captured. A number of slightly different numerical definitions for the resolution exist. For the lateral resolution (i.e. the resolution in the image plane), the Rayleigh criterion is commonly used (Pawley, 2006),

$$
d_{\mathrm{min}}^{\mathrm{lat}}=\frac{1.22 \cdot \lambda_{\mathrm{em}}}{2 \cdot N A}
$$

with $\lambda_{\mathrm{em}}$ being the wavelength of the emission light. It holds both for widefield and confocal microscopes, unless the confocal pinhole is smaller than $0.25 \mathrm{AU}$. The 

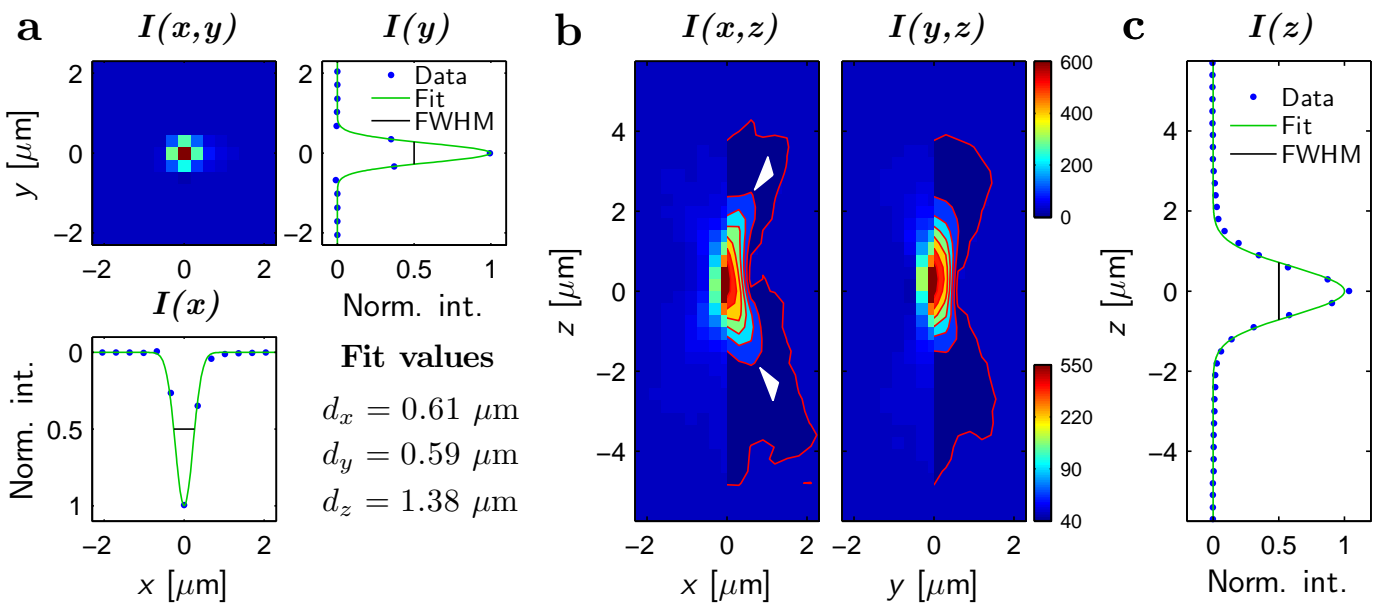

Figure 3.4: Point spread function using the $63 \times$ Achroplan $0.95 \mathrm{~W}$ objective. For a quantification, analytical functions were fitted to the intensity profiles of individual beads. For display purpose, the image stacks were aligned using the bead center obtained from the fit, and averaged. Shown are the intensity profiles through the center of the bead as 2D images and 1D plots. (a) Intensity profile in the $(x, y)$-plane and along the $x$ - and $y$-axis. Shown are the data points (blue) and fitted Gaussians (green). The black line indicates the full width at half maximum (FWHM) of the fitted curve, which was used for the quantification of the width of the PSF. The lateral profiles show a symmetric intensity distribution along the principle axis, with virtually identical FWHM. Listed are the fit values for the three spatial directions. See Table 3.2 for the respective values of other objectives and standard deviations. (b) The intensity profiles in the $(x, z)$ - and $(y, z)$-planes are symmetric with respect to the optical axis. Therefore, two types of visualization could be combined in each graph. The averaged intensity values are shown in the left half of each image (top colorbar), the right half shows superimposed a logarithmic contour plot for better visualization of the low intensities (bottom colorbar). The slit aperture results in different profiles in these two planes, with cone-like side lobes in the $(x, z)$-plane (white arrowheads), indicating a reduced rejection of out-of-focus light. (c) Axial intensity profile with fitted Gaussian and FWHM.

anisotropy of the beam path is thus not expected to have an effect on the lateral resolution. The axial resolution of a conventional point-scanning confocal microscope with a pinhole diameter larger than $0.25 \mathrm{AU}$ is given by (Wilhelm et al., 2003)

$$
d_{\min }^{\mathrm{ax}}=\frac{0.88 \cdot \lambda_{\mathrm{exc}}}{n-\sqrt{n^{2}-N A^{2}}},
$$

with $\lambda_{\text {exc }}$ being the wavelength of the excitation light. While these definitions specify a theoretical lower bound, a given confocal microscope might not reach these limits. 
A common reason is the insufficient illumination of the backfocal plane, which leads to a smaller effective $N A$. Aberrations or misalignment of optical elements can further reduce the resolution of an imaging system (Pawley, 2006; Wilhelm et al., 2003). Additionally, the axial resolution of the LIM might be different from a conventional confocal microscope for which Equation 3.4 was derived, as the illumination with a line instead of a point can be expected to decrease the optical sectioning capabilities (Pawley, 2006). The sampling theorem by Shannon adds another fundamental limitation to the resolution of a measuring system (Pawley, 2006; Shannon, 1949). When sampling a continuous, one-dimensional signal, the highest frequency that is contained in the discrete measurements is half the sampling frequency (or expressed in spatial coordinates: the smallest distance that can be resolved is twice the distance between sampling points). The sampling frequency for the $x$-direction is fixed by the size of the CCD pixels (scaled with respect to the object plane, see Tab. 3.1). For the $y$-direction, the same sampling interval is chosen in order to create square pixels, while the sampling rate in $z$-direction is only limited by the precision of the piezo actuator, thus not posing an effective limit to the resolution.

Since all of these factors have an effect on the resolution of an imaging system, it is necessary to determine the resolution of the LIM experimentally.

\section{Determination of the resolution}

A common way to measure the resolution of a microscope is by imaging fluorescent beads with a diameter significantly below the theoretical resolution limit. Since optical systems behave over a large range linearly with respect to signal intensity, the image of such bead can be understood as the 'impulse response function' of the microscope. In microscopy this function is referred to as the point spread function (PSF) and it characterizes the imaging properties of the optics (in the linear approximation). While a comprehensive characterization requires the complete threedimensional intensity distribution of the PSF, the intensity profiles along the three principles axis can be used to assess the resolution of the microscope along its three principle directions. The resolution along the $x$-, $y$ - and $z$-axes can be obtained from fits of appropriate functions to the intensity profiles along these axes. The lateral 
intensity distribution of the PSF can be described by a squared Bessel function, the axial profile by a sinc-function. For the sake of determining the width of these profiles, Gaussian functions can be used instead, as they describe the profile sufficiently well ((Pawley, 2006) and Materials and Methods for details of fitting procedure).

Measurements of PSFs are very susceptible to noise, especially due to the fast drop of intensity toward the periphery of the profile. It is thus necessary to acquire large numbers of 3D image stacks of single beads. For each of these image stacks, the center of the PSF is determined as the 'center of gravity' of the light intensities. A two-dimensional Gaussian is then fitted to the intensity profile through this center. The results of this fit yield the full width at half maximum (FWHM) values for the $x$ - and $y$-direction. The axial intensity profile through the image center is fitted by a one-dimensional Gaussian, yielding the FWHM for the z-direction. For display purposes, the results of the fits can be used to align and average the individual image stacks, creating a representative visualization of the PSF for a given objective.

The PSFs of the objectives used throughout this thesis were acquired and analyzed $(25 \times, 40 \times$ and $63 \times$, see Materials and Methods for details). Figure 3.4 shows the following results for the $63 \times$ objective as an example: the averaged intensity distributions in the $(x, z)$-, $(y, z)$ - and $(x, y)$-plane, the averaged profiles along the $x-, y$ - and $z$-axes as well as the fitted functions and the mean of their FWHM. The mean values and standard deviations of the FWHM are listed in Table 3.2 for the three objectives. 
Table 3.2: Fit results and theoretical limits for the FWHM of the PSFs along the three spatial coordinates for various objectives. The fit values are the results of fitting Gaussian functions, as shown in Figure 3.4 for the $63 \times$ objective. Theoretical limits are calculated according to the equations (3.3) and (3.4) for lateral and axial resolution, respectively. The pixel pitch imposes an additional limit due to the resulting sampling rate. This limit is given by twice the pixel size relative to the object plane, as specified for each objective in $\mu \mathrm{m}$ and Airy units in the first column. Number of beads: $25 \times: n=42,40 \times: n=25,63 \times: n=50$.

\begin{tabular}{ccccc}
\hline $\begin{array}{c}\text { Objective } \\
(\text { Pixel pitch })\end{array}$ & $\begin{array}{c}\text { Dimension } \\
{[\mu \mathrm{m}]}\end{array}$ & $\begin{array}{c}\text { Fit } \\
\text { values }\end{array}$ & $\begin{array}{c}\text { Theoretical } \\
\text { limit }\end{array}$ & $\begin{array}{c}\text { Sampling } \\
\text { limit }\end{array}$ \\
\hline $\mathbf{2 5} \times / \mathbf{0 . 8} \mathbf{W}$ & $x$ & $1.58 \pm 0.16$ & 0.39 & 1.80 \\
$(0.90 \mu \mathrm{m} / 1.18 \mathrm{AU})$ & $y$ & $1.48 \pm 0.15$ & 0.39 & 1.80 \\
\hline & $z$ & $4.22 \pm 0.66$ & 1.61 & - \\
\hline $\mathbf{4 0 \times / 0 . 8 ~ W}$ & $x$ & $1.07 \pm 0.12$ & 0.39 & 1.08 \\
$(0.54 \mu \mathrm{m} / 0.73 \mathrm{AU})$ & $y$ & $1.03 \pm 0.11$ & 0.39 & 1.08 \\
\hline & $z$ & $2.26 \pm 0.28$ & 1.61 & - \\
\hline $\mathbf{6 3} \times / \mathbf{0 . 9 5} \mathbf{W}$ & $x$ & $0.61 \pm 0.05$ & 0.33 & 0.60 \\
$(0.30 \mu \mathrm{m} / 0.55 \mathrm{AU})$ & $y$ & $0.59 \pm 0.04$ & 0.33 & 0.60 \\
\hline
\end{tabular}

\section{Lateral resolution}

The symmetry of the intensity profile of the PSF in the $(x, y)$-plane (Fig. 3.4a) indicates that the resolutions in $x$ - and $y$-direction are identical for the $63 \times$ objective. This is confirmed by the fit results. For each of the three objectives, the difference of the FWHM in $x$ - and $y$-direction lies within the variance of the measurements (3.2). The anisotropic image acquisition does thus not affect the lateral resolution. The lateral FWHM are significantly larger than limits calculated according to Equation 3.3. This discrepancy is mainly due to the low sampling rate. Based on the pixel pitch with respect to the object plane (first column in Tab. 3.2), the resolution limit imposed by the sampling rate can be calculated for each objective (last 
column in Tab. 3.2). For the $40 \times$ and $63 \times$ objectives, these theoretical values lie within the error interval of the measurements. For the $25 \times$ objective, the measured FWHM is slightly smaller than the theoretical limit. This can be explained by the fact that unlike the resolution limit, the FWHM of a PSF is not scaling linearly with the sampling distance, especially for large differences between object size and sampling distance. The lateral resolution of the LIM is thus limited by the sampling rate, which is defined by the pixel pitch of the CCD camera and the magnification factor from the object to the image plane. It should be noted that imaging systems sample the space anisotropically by creating a pixel (or voxel) grid with an arbitrary orientation to the specimen. The resolution in directions other than the principle directions defined by this grid is slightly lower, due to larger sampling intervals(Pawley, 2006).

\section{Axial resolution}

A comparison of the intensity profiles in the $(x, z)$ - and $(y, z)$-plane illustrates the effect of the anisotropy introduced by the slit aperture (Fig. 3.4b). The profile in the $(x, z)$-plane, which is parallel to the direction of the slit, shows side lobes in the logarithmic contour plot (white arrowheads in Fig. 3.4, (Pawley, 2006)). These resemble the PSFs measured using confocal microscopes with large pinhole diameters or widefield microscopes. In contrast, the profile in the $(y, z)$-plane, perpendicular to the direction of the slit, shows the ellipsoid contours typical for a confocal microscope with a small pinhole diameter $(\leq 1 \mathrm{AU})$. This profile demonstrates the rejection of out-of-focus light by the confocal aperture which is the basis for optical sectioning. The FWHM values are larger than the theoretical limit calculated for a point-scanning confocal microscope. The difference between the measured values and the theoretical limits can have different reasons. The described effect of the one-dimensional aperture reduces the axial resolution (Pawley, 2006). Further, an insufficient illumination of the objective's backfocal plane will reduce its effective $N A$. In the LIM, the laser beam is focussed into a line in the backfocal plane. Despite the effort to increase the length of this line, the Gaussian profile of the laser beam results in an intensity drop towards the periphery of the line. An effect on 
the resolution can thus not be excluded. Further, as the objectives cover a large field of view, aberrations by the optical system can be an additional cause for an increase in the axial FWHM. The limit given by Equation 3.4 is calculated for optimal imaging conditions, which are only satisfied for the vicinity of the optical axis. Imaging quality is typically reduced in the periphery of the field of view. Another source for the deviation of the measurement from the theoretical limit is the low lateral sampling rate, which is imposed by the pixel pitch of the camera. Due to this undersampling, each value of the axial intensity profile is an average over a significant area in the $(x, y)$-plane. As the width of off-axis profiles through a bead's image increases with the distance from the optical axis, this results in a broadening of the recorded axial profile. The differences between the theoretical limits and the actual values are largest for the low magnification $25 \times$ objective. However, even for this objective, the axial FWHM of the PSF is significantly smaller that the typical diameter of a single neuron, enabling observation of somata without strong overlap of signals emitted by different cells. Test measurements with thin fluorescent layers confirmed these results (data not shown). The width of the PSFs along the $z$-direction is an indicator for the thickness of the optical section. It can serve as a guide for the optimal separation of $z$-planes for 3D imaging and for the choice of an objective for a given experiment. Only for extensively fluorescing specimens, the optical sectioning might be reduced due to the side lobes in the $(x, z)$-profiles of the PSFs (Fig. 3.4c).

The obtained values are summarized in Table 3.2 and serve as a guideline for which objective is appropriate for the application of interest.

\subsubsection{Acquisition speed for 2D- and 3D-imaging}

\section{Acquisition speed for $2 D$ imaging}

The acquisition speed of a laser scanning microscope with mechanical (non-resonant) scan mirrors is limited by the inertia of the oscillating mirrors (Pawley, 2006; Tan et al., 1999). CCD-camera based systems on the other hand are limited by the read-out speed of the camera (see e.g. Appendix 3 in (Pawley, 2006)). The LIM combines 1D scanning with a fast CCD sensor, it is thus not obvious which factor 


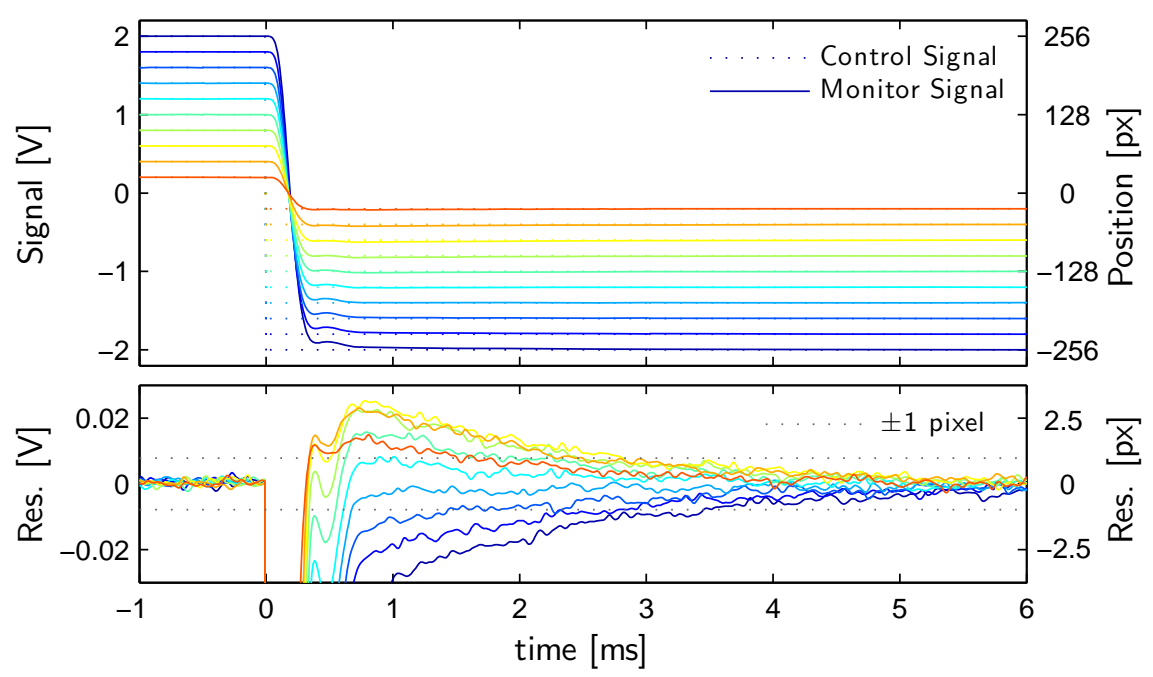

Figure 3.5: Response behavior of the scan mirror to voltage steps of different amplitude. The control signal (top, broken lines) is a step function, the voltage applied to the mirror and the corresponding pixel position are indicated by the left and right $y$-axes, respectively. The monitor signal of the mirror (solid lines) shows a steep shoulder and, depending on the amplitude of the control signal, a slight overshoot or a creep behavior for small and large amplitudes, respectively (bottom: residuals). The control system is optimized for medium frames sizes (light blue curves; see text for details), for which the residuals are smaller than one pixel width after about $0.5 \mathrm{~ms}$ (bottom graph, pixel width of the camera indicated by broken lines).

will be limiting for the acquisition speed of this microscope.

The acquisition time $t_{\mathrm{Acq}}^{\mathrm{Line}}$ for a single line is the sum of the exposure time $t_{\mathrm{Exp}}^{\mathrm{Line}}$ and the constant read-out time of the camera of approximately $7 \mu \mathrm{s}$. Since the read-out of each line is triggered in order to be synchronous to the scan mirror movement, $t_{\text {Acq }}^{\text {Line }}$ is restricted to multiples of the length of the clock cycle provided by the control unit PC $(32 \mu \mathrm{s})$. The minimum frame acquisition time $t_{\text {Acq }}^{\text {Frame }}$ additionally depends on the number of lines that a frame is composed of. While in $x$-direction always the complete line (i.e. the 512 pixel the sensor is composed of) has to be read out, the size of the $y$-dimension can be selected by the user, with a maximum of 512 lines per frame. $t_{\text {Acq }}^{\text {Frame }}$ can then be calculated as the product of $t_{\text {Acq }}^{\text {Line }}$ and the number of lines $N_{\text {Acq }}$. It is thus necessary to investigate whether the scan mirror is fast enough to achieve the respective scan frequencies $f_{\text {Acq }}^{\text {Frame }}=1 / t_{\text {Acq }}^{\text {Frame }}$. 

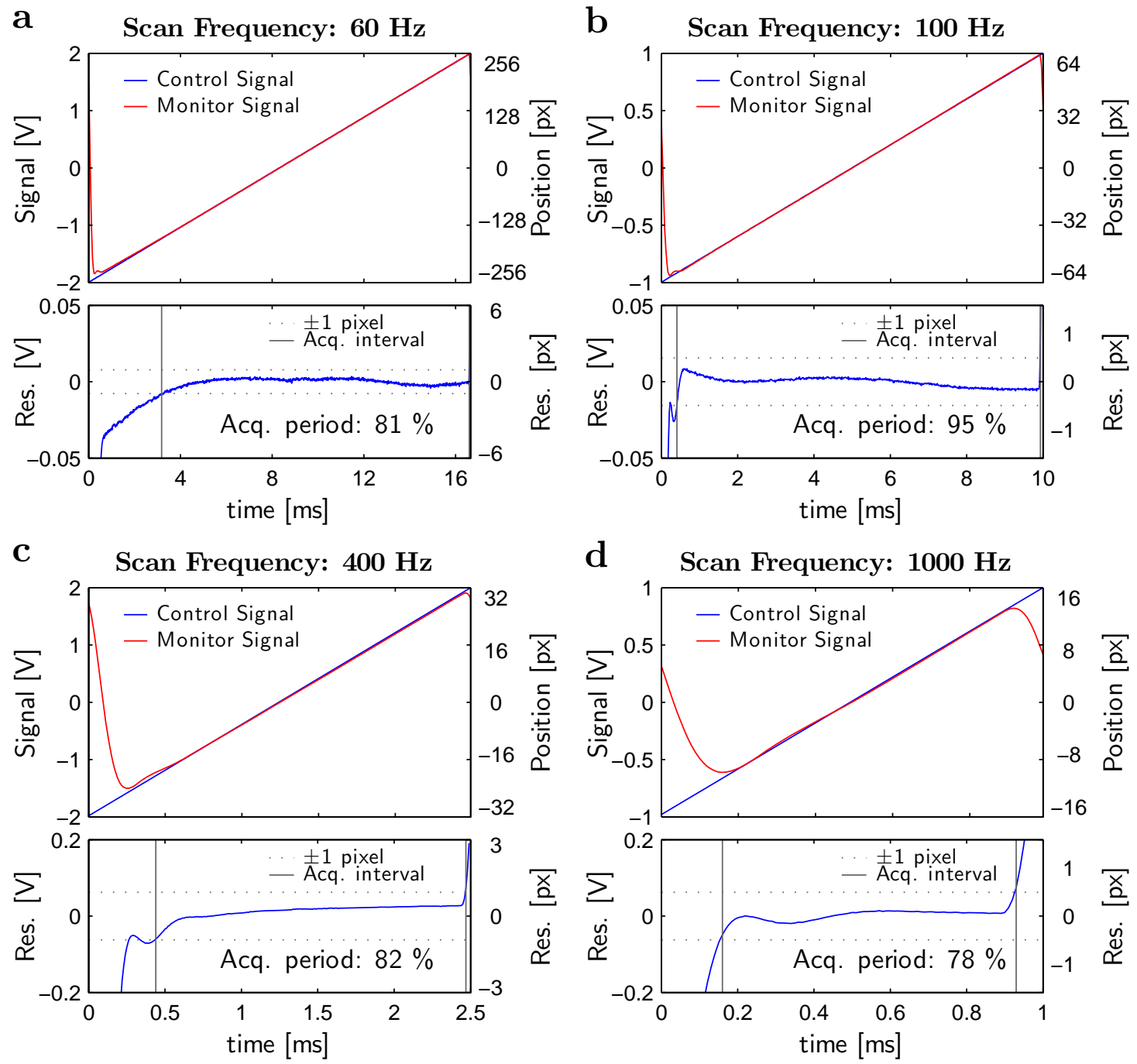

Figure 3.6: Response behavior of the scan mirror to voltage ramps of different frequencies and amplitudes. The depicted scan schemes are test charts used to determine which fraction of a scan cycle can be used for data acquisition. Due to the addition of pre-acquisition lines, the effective frame rates differ from the values indicated in these graphs. Table 3.3 lists effective frame rates for a variety of experimental parameters. Shown are four examples of scanning parameters relevant to experiments in this thesis (top: control and monitor signal; bottom: residual). $\pm 1 \mathrm{~V}$ corresponds to a frame width of $1 / 2$ frame height, $\pm 2 \mathrm{~V}$ corresponds to a square full frame. The best response is achieved for medium scan amplitudes and frequencies (b), where more than $90 \%$ of the cycle can be used for data acquisition. For large amplitudes (a) or very fast scan rates (c and d), only about $80 \%$ of the cycle can be used for acquisition (percentages for all protocols indicated in graphs). Note that for $(\mathrm{b})-(\mathrm{d})$ the pixel spacing in $y$-direction is larger than in $x$-direction (pixel aspect ratio $\Delta x / \Delta y \neq 1$ ). This reduces noise (longer exposure times for fewer pixel) by sacrificing spatial resolution. The scan scheme shown in (b) was used for most of the experiments in section 3.3, the scan protocol in (c) for the characterization of the perfusion system (Fig. 3.15b). 
Table 3.3: Recommended number of lines $N_{\text {Tot }}=N_{\text {Acq }}+N_{\text {Pre }}$ and resulting effective frame acquisition rates $f_{\mathrm{Acq}}^{\text {Frame }}$ for different scan parameters (number of acquisition lines $N_{\mathrm{Acq}}$, line acquisition time $t_{\operatorname{Exp}}^{\mathrm{Line}}$ and pixel aspect ratio $\left.\Delta x: \Delta y\right)$. These values result from the response curves of the scan mirror at the respective scan speeds as shown in Figure 3.6 and serve as a guideline for the design of scan protocols.

\begin{tabular}{ccccc}
\hline$N_{\text {Acq }}$ & $t_{\text {Acq }}^{\text {Line }}[\mu \mathrm{s}]$ & $\Delta x: \Delta y$ & $N_{\text {Tot }}$ & $f_{\text {Acq }}^{\text {Frame }}[\mathrm{Hz}]$ \\
\hline 32 & 32 & $1: 8$ & 39 & 800 \\
64 & 32 & $1: 8$ & 75 & 410 \\
64 & 64 & $1: 2$ & 68 & 230 \\
128 & 32 & $1: 1$ & 148 & 210 \\
128 & 64 & $1: 2$ & 134 & 117 \\
256 & 32 & $1: 1$ & 269 & 117 \\
256 & 64 & $1: 2$ & 304 & 50 \\
512 & 32 & $1: 1$ & 609 & 50 \\
\hline
\end{tabular}

The scan signal, which is provided by the control PC, is a stair-case like curve with variable amplitude and frequency. The scan mirror is controlled by a proportional - integral - derivative controller (PID controller), which has to be tuned to achieve optimal performance in response to a given command signal (Tietze and Schenk, 1999). Different experiments require different scan patterns in order to adjust the field of view (i.e. the amplitude of the scan signal) or temporal resolution (i.e. the frequency of the signal). When tuning the PID, a compromise had to be made to ensure good properties for the most common experimental conditions. In the following, the results of this tuning procedure will be presented for different scan parameters.

First, the amplitude dependence of the scan mirror behavior was examined. To this end voltage steps of varying amplitude were used as the command signal, and the position signal of the scan mirror (provided from the PID controller) was recorded (Fig. 3.5). The largest voltage step in Figure 3.5 corresponds to a full frame $(512 \times 512$ pixel $)$. The plot of the residuals between command and position signal 
shows that for the (most commonly used) amplitudes of one half or one quarter of a full frame, the error is less than one pixel after about $0.5 \mathrm{~ms}$. For all shown amplitudes, the command position is reached within less than $3.5 \mathrm{~ms}$ with a precision of one pixel width. The different shapes of the responses and the corresponding residuals demonstrate that the PID controller is not behaving linearly and that a precise adjustment to the correct range of parameters is important.

In order to evaluate the scan mirror behavior under the experimental conditions, voltage ramps of different amplitudes and frequencies were applied while measuring the response of the mirror. While it is possible to improve scan mirror behavior by optimizing the waveforms to the physical properties of the galvanometer (Tan et al., 1999), it was tested whether simple voltage ramps can yield sufficiently precise mirror movements for the required one-dimensional scanning procedure. Figure 3.6 shows a number of representative conditions which relate to actual experiments demonstrated in this thesis. For easier comparison between command and monitor signal, all curves are corrected for phase shift by aligning the zero-crossing of the two curves. Since this implies only a constant shift in time for a given curve it does not affect the interpretation of the data. As expected from Figure 3.5, for none of the cases the mirror can follow the command signal perfectly. It lags behind the sharp up-rise and subsequently minimizes the difference to the command signal.

The design of the scan protocol allows the introduction of an arbitrary number of 'pre-acquisition lines' which precede the start of measurement in each scan cycle (Fig. 2.2). Strong deviations of the position from the command signal can be avoided in this way for the acquisition period. The question remains whether the mirror follows the command signal reasonably well for the majority of the scan period. Deviations affect the acquired image in form of a deformation in $y$-direction (i.e. non-uniform pixel width across the frame). While excessive deformation should be avoided, a systematic error in the range of $1-2$ pixel can be accepted, especially since high-speed imaging is usually not combined with detailed morphological measurements. Figure 3.6 shows that even for large amplitudes (Fig. 3.6a) and high frequencies (Fig. 3.6d) the accuracy of the mirror movement is satisfactory over approximately $80 \%$ of the scan period. Medium scan speeds (around $100 \mathrm{~Hz}$ frame 
rate) and amplitudes (1/2 full frame) yield the best results and more than $90 \%$ of the scan period can be used for data acquisition. In order to maximize the frame rate, measurements like the examples in Figure 3.6 can be used to determine the minimum number of pre-acquisition lines. Table 3.3 gives some guideline numbers for different experimental conditions together with the resulting maximum frame rates. These numbers demonstrate that the minimum line acquisition time of $32 \mu \mathrm{s}$ is the limiting factor for the acquisition speed. It is therefore not necessary to use more complex command signals to reduce fly-back times (Tan et al., 1999).

It should be mentioned that the maximum frame rate is only achieved for the shortest possible exposure time provided by the camera $(25 \mu \mathrm{s})$. This time is in many practical cases insufficient given the low intensity emanating from most living biological samples. Especially for experiments that require the acquisition of a large number of frames, the excitation intensity has to be reduced to prevent excessive photo bleaching. Consequently, the experimenter is often forced to raise the frame acquisition time above the technical limit.

\section{Acquisition speed for $3 D$ imaging}

3D imaging is enabled using a piezo driven actuator to change the position of the objective along the optical axes. The piezo drive is regulated by a proportional integral controller (PI controller, (Tietze and Schenk, 1999)). While the physical properties of the scan mirror are fixed, the objective can - and often has to - be changed, resulting in different response behavior to identical scan schemes. This difference can be severe when objectives have very different masses. The optimal setting of the PI strongly depends on the inertia of the controlled system, leading to overshoot, oscillations or damping if not properly tuned. Increasing the gain leads to a faster response, but can also result in oscillations (proportional gain) or overshoot (integral gain) when the inertia of the controlled system is too high (Tietze and Schenk, 1999). On the other hand, lowering the gain decreases the responsiveness of the system but can show improved behavior for systems with a large inertia. Therefore the responses of both 'heavy' and 'light' objectives (the ZEISS Plan-Apochromat $25 \times \mathrm{W} / 0.8$ and the ZEISS Achroplan $40 \times \mathrm{W} / 0.8$, respectively) was examined for 

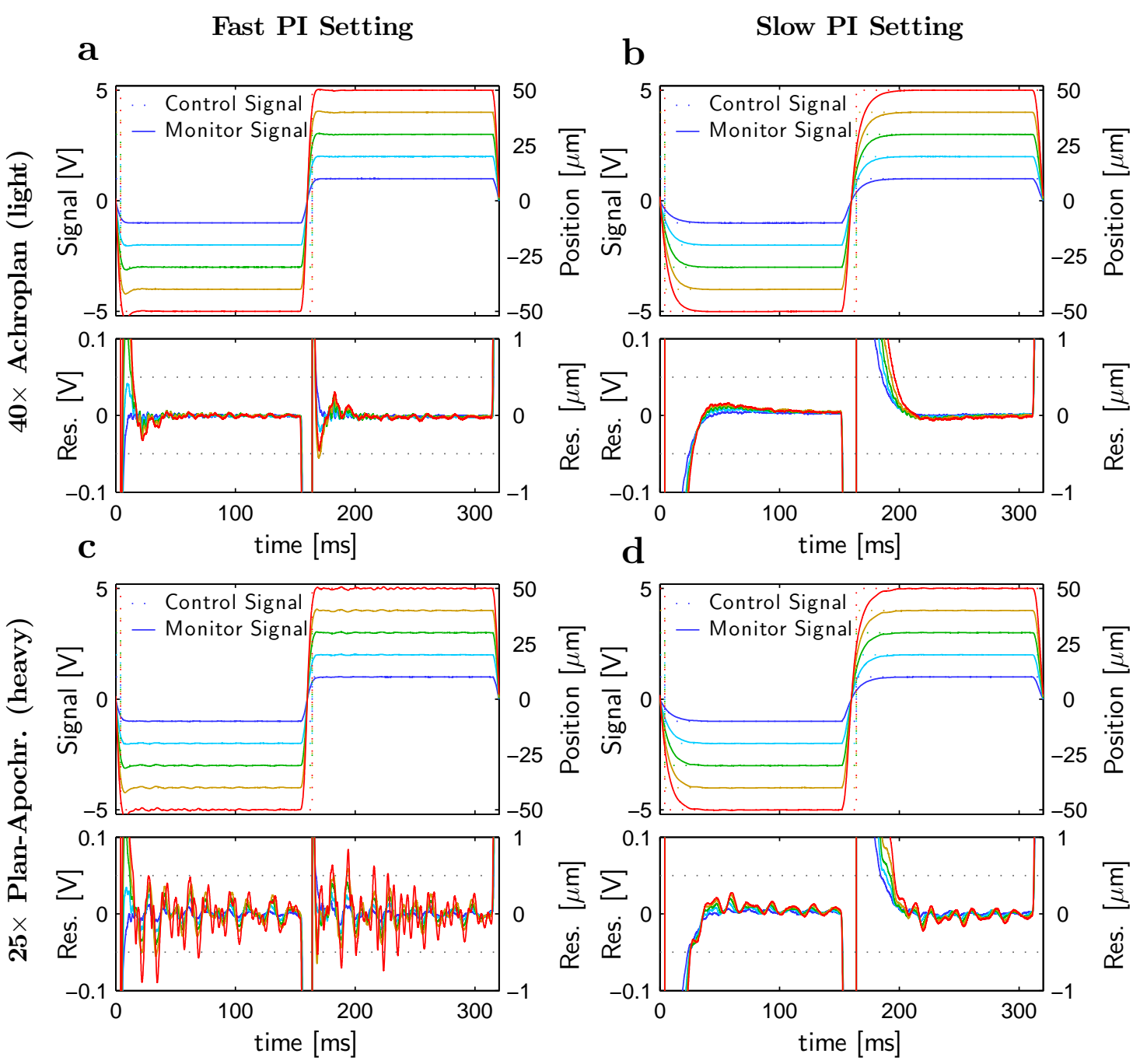

Figure 3.7: Response behavior of the objective's piezo actuator to voltage steps of different amplitudes. The position of the objective was monitored for both light and heavy objectives (first and second row, respectively), and using both fast and slow settings of the PI (first and second column, respectively). Top: control and monitor signal. The corresponding command voltage is indicated by the line colors. Bottom: residual and $\pm 0.5 \mu$ m indicated by broken lines for comparison between measurements. (a) The light objective shows ideal behavior when used with the fast PI setting, with only a small overshoot and fast decaying oscillations. The residual is less than $0.5 \mu \mathrm{m}$ after less than $15 \mathrm{~ms}$ even for the largest amplitude of $\pm 50 \mu \mathrm{m}$. (b) Using the slow PI settings with the light objective introduces a slow creep, which reduces the responsiveness of the system. Residual below $0.5 \mu \mathrm{m}$ are only achieved after about $30 \mathrm{~ms}$, even for small amplitudes. (c) Using the heavy objective with the fast PI settings results in strong and slowly decaying oscillations, making this combination unsuitable for experiments. (d) Combining the heavy objective with slow PI settings reduces the amplitude of the oscillations to an acceptable level. 
a

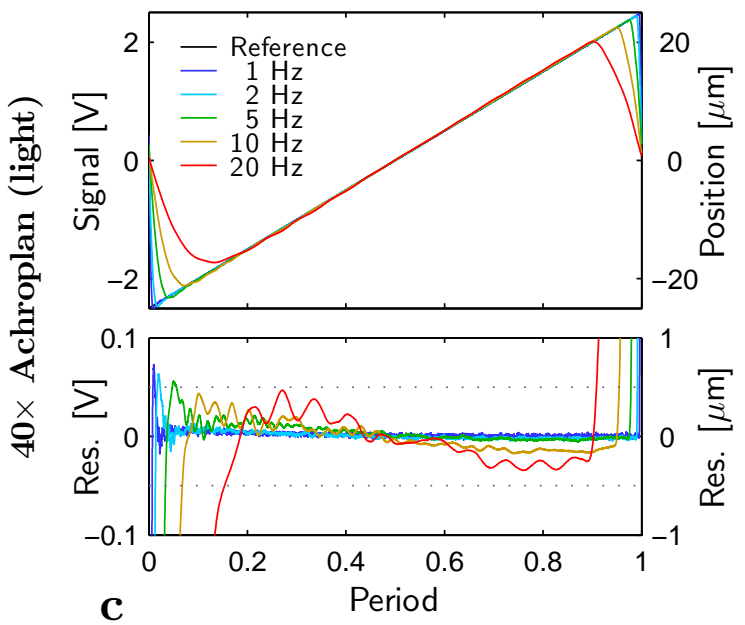

Fast PI Setting

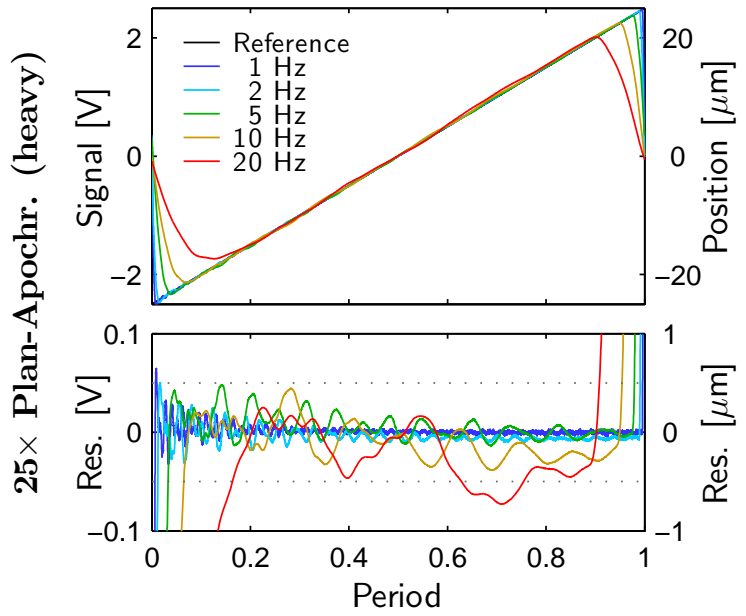

b

Slow PI Setting
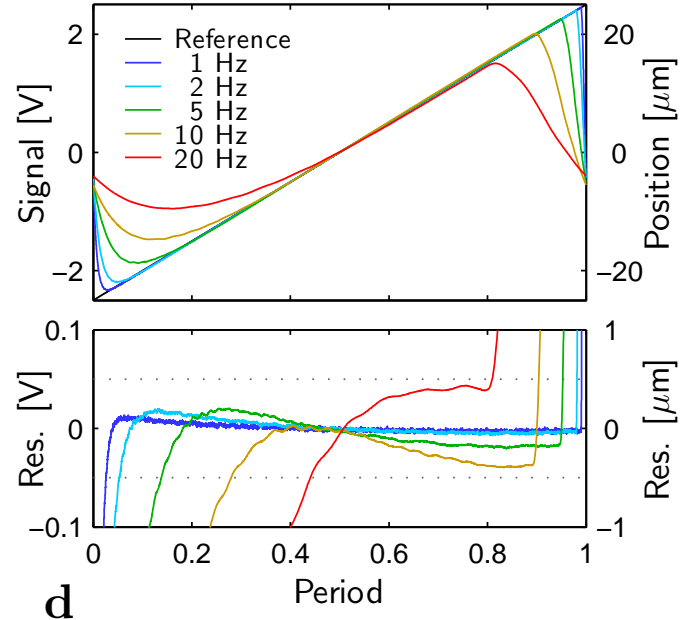
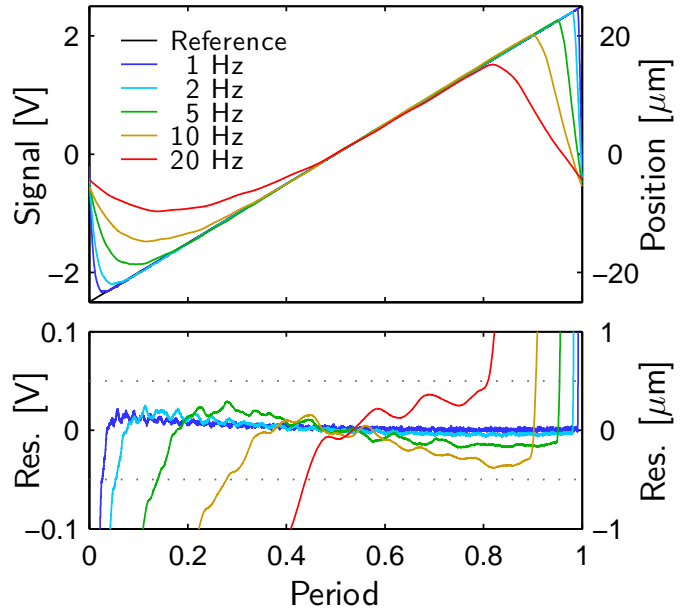

Figure 3.8: Response behavior of the piezo actuator that drives the objective at different frequencies for a fixed amplitude. Top: control and monitor signal. Bottom: residual and $\pm 0.5 \mu$ m indicated by broken lines for comparison. The time is scaled to one scan period to allow a direct comparison of different scan frequencies. (a) Similarly to the responses to voltage steps (Fig. 3.7), the light objective is best used with the fast PI setting. More than $90 \%$ of the scan cycle can be used for data acquisition for frequencies up to $10 \mathrm{~Hz}$. (b) Using the slow PI setting for the light objective reduces the responsiveness of the system significantly (compare to (a)). For most scan rates a large fraction of the cycle cannot be used for data acquisition. (c) The heavy objective combined with the fast PI setting shows large oscillations which degrade image quality. (d) Using the slow PI settings for the heavy objective reduces the amplitude of the oscillations and scan frequencies up to $5 \mathrm{~Hz}$ can be achieved with about $80 \%$ of the cycle available for imaging. 
different settings of the PI.

To evaluate the amplitude dependence of the system's responsiveness, the response to voltage steps is shown in Figure 3.7 for both 'fast' PI settings (i.e. larger gains) and 'slow' PI settings (i.e. smaller gains). In contrast to the scan mirror, the controller of the piezo actuator behaves approximately linearly, resulting in qualitatively similar residuals for different amplitudes (compare Fig. 3.5 and 3.7). This implies that larger scan amplitudes will be accompanied by larger residuals.

Using a light objective and the fast PI settings resulted in a rapid decrease of the difference between command and monitor signal, allowing for high scan frequencies in $z$-direction. Using the slow PI settings on the other hand, it took significantly longer to reach the command position, severely limiting the potential scan speed. While the heavy objective combined with slow settings showed a reasonably good response (as fast as possible given the large weight of the objective), it exhibited strong oscillations when used with the fast setting. In order to overcome either limitation, two sets of PI controllers were installed onto the control board, enabling the user to switch between 'fast' and 'slow' settings depending on the objective, scan amplitude and scan frequency in z-direction.

Figure 3.8 shows the response of the system to voltage ramps as they are used for the acquisition of $z$-stacks in actual experiments. Similarly to the scan mirror, the control software allows to introduce a certain time delay (in multiples of frames) at the beginning of each stack. In this way the return time of the objective can be excluded from the measurement (see Fig. 2.2). As expected from the experiments using voltage steps (Fig. 3.7), the fast setting is optimal for the light objective and allows precise movements up to $10 \mathrm{~Hz}$ after an initial time delay (about $10 \%$ of the scan period, Fig. 3.7a). The residuals are below $0.5 \mu \mathrm{m}$. Since this is less than the axial resolution for all tested objectives (see Fig. 3.4 and Tab. 3.2), it does not have a significant impact on the image quality. For the heavy objective, the highest usable frequency is $5 \mathrm{~Hz}$, where about $80 \%$ of the cycle can be used for image acquisition (Fig. 3.7d). 


\subsubsection{Discussion of the microscope setup}

The idea to modify the concept of a confocal point-scanning system into a linescanning microscope with a slit aperture was first introduced by Maurice (1974). The imaging properties of confocal microscopes with slit apertures were quantitatively discussed by Sheppard and Mao (1988). Several realizations of this approach have since been described (Engelmann, 2006; Im et al., 2005; Masters and Thaer, 1994; Poher et al., 2008). Interestingly, there is few literature addressing biological questions with such devices. In many cases, non-fluorescent (Masters and Thaer, 1994; Maurice, 1974) or non-biological (Im et al., 2005; Poher et al., 2008) applications were used for the demonstration of the capabilities of these microscopes. In order to addressing biological questions with fast imaging of fluorescent indicator dyes, it is necessary to meet various requirements. High spatial resolution, high temporal resolution, long observation times, repeated trials of a given experiment to enable statistical evaluations, high signal-to-noise ratio, no photo-bleaching of the dye and no effect on the biological preparation are some of the desired properties. These requirements are however mutually restricting, mainly due to the limitations in fluorescent microscopy using living specimen. Besides the technical difficulty of achieving fast frame rates, increasing the acquisition rate implies an increase of acquired images for a given observation time. The acquisition of large numbers of frames is typically accompanied by photo-bleaching, which results in a decrease of signal over time and can additionally result in the production of toxic substances (Knight et al., 2003). In fact, fluorescent dyes have a statistical 'life-time' in terms of excitation cycles (typically $10^{4}$ to $10^{5}$ per dye molecule). This poses a strict physical limit on the number of frames that can be acquired without using exaggerated dye concentrations. Experiments using fluorescent dyes thus always face a trade-off between various experimental parameters.

The current implementation of a line-scanning confocal microscope was thus designed to optimize temporal resolution and signal efficiency, slightly compromising flexibility (no adjustable pinhole) and spatial resolution. In this way, the setup is optimized for addressing biological questions that demand a high temporal resolution. 
Both lateral and axial resolution suggest that small structures (such as fine dendritic branches or axons) ares adequately sampled using the $63 \times$ objective (and presumable higher magnification / higher $N A$ objectives). The ability to resolve these structure is limited for the $25 \times$ and to a smaller extent for the $40 \times$ objective. This does not exclude the detection of these processes, but it rather limits the discrimination of closely positioned fine structures. Obviously, the spatial resolution could have been improved by choosing a different design of the optics, i.e. by increasing the magnification from the object plane to the image plane. However, the current configuration was chosen to distribute the emission light over a smaller number of pixels. As the read-out of each pixel adds noise to the data, the reduction of the pixel number can be described as a pre-read-out binning. This results in an increase of the signal-to-noise ratio at the cost of spatial specificity. Additionally, the field of view is enlarged by the smaller magnification, which is beneficial for the investigation of neuronal networks.

The LIM shows a highly increased acquisition speed when compared to conventional laser-scan or widefield microscopes. The scan mirror behavior was optimized for scan amplitudes that correspond to half the length of the linear CCD array and to frame rates around $100 \mathrm{~Hz}$ (compared to typically $1-10 \mathrm{~Hz}$ for conventional confocal microscopes). This gain in speed can for example be used for fast $2 \mathrm{D} \mathrm{Ca}^{2+}$ imaging, with a sampling frequency above the average spike rate of many types of neurons, in particular the principle neurons of the Xenopus olfactory bulb (Scheidweiler et al., 2001). This should even enable the detection of single action potentials from the time course of $\mathrm{Ca}^{2+}$-sensitive dyes (Lin et al., 2007; Smetters et al., 1999). Alternatively, the increased acquisition speed can be used to acquire complete image stacks at up to $10 \mathrm{~Hz}$. This resolution is sufficient for the observation of the neuronal activity patterns, as it has been done at comparable or even lower acquisition rates in 2D imaging for many years. Thanks to the confocality of the microscope, the investigation of these time courses is not restricted to somata, but can be extended to neuronal processes across the observed volume. It is thus possible to investigate activity patterns of a large fraction of a given neuronal network with high spatial and temporal resolution. 


\subsection{Activity Correlation Imaging (ACI)}

\subsubsection{The general principle of activity correlation imaging}

Analysis of neuronal network function would be drastically improved by a technique that allows the simultaneous measurement of both the activity of every neuron in a network and its morphology and projection pattern. Significant advances in one or the other direction have been made recently. On the one hand, transgenic strategies for combinatorial expression of fluorescent proteins allow the visualization of the detailed morphology of neuronal populations with excellent contrast (Livet et al., 2007) but fail to detect the functional activities of the labeled neurons. On the other hand, functional imaging using bolus loading of membrane-permeable $\mathrm{Ca}^{2+}$ indicators allows an almost complete visualization of neuronal activity at cellular resolution (Ohki et al., 2005, 2006; Stosiek et al., 2003). However, because this technique unspecifically stains every neuron in the network, it does not give sufficient contrast to visualize the fine processes of individual neurons (Garaschuk et al., 2006; Nagayama et al., 2007). Alternatively, $\mathrm{Ca}^{2+}$ indicators can be loaded into a sparse subset of neurons to enable a simultaneous visualization of activity and fine neuronal structures (Nagayama et al., 2007), but this approach is intrinsically limited to image a small fraction of cells. Combining the advantages of these approaches would provide an invaluable tool for neuroscience research.

A new method is proposed that allows high contrast visualization of neuronal circuitry during large-scale functional imaging experiments using bolus loading of membrane permeable $\mathrm{Ca}^{2+}$ indicators. Specifically, image contrast is derived from the diversity of temporal activities recorded with $\mathrm{Ca}^{2+}$ sensitive dyes, rather than from the differences in fluorescence intensities. This allows to visualize individual fine neuronal processes even when the entire neuronal circuit is unspecifically stained with fluorescence dye. This method develops its full potential when combined with the fast image acquisition provided by the LIM and provides a multi-color visualization of neuronal networks during functional imaging of brain tissue. The spontaneous $\mathrm{Ca}^{2+}$ activities in the mitral/tufted $(\mathrm{M} / \mathrm{T})$ cell layer of the olfactory bulb (OB) of Xenopus laevis tadpoles were imaged $(25 \times 0.8 \mathrm{~W}$ objective $)$ using bolus loading 
a

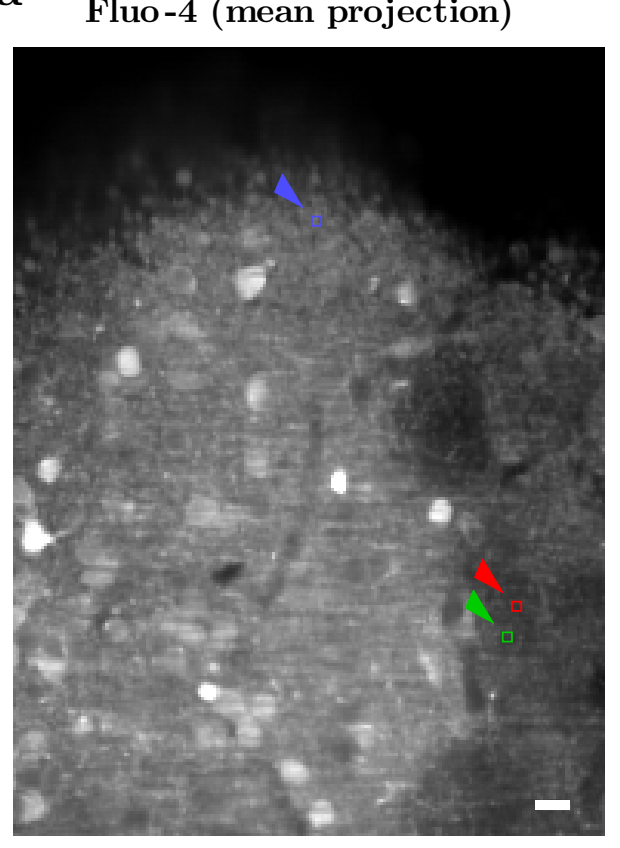

b

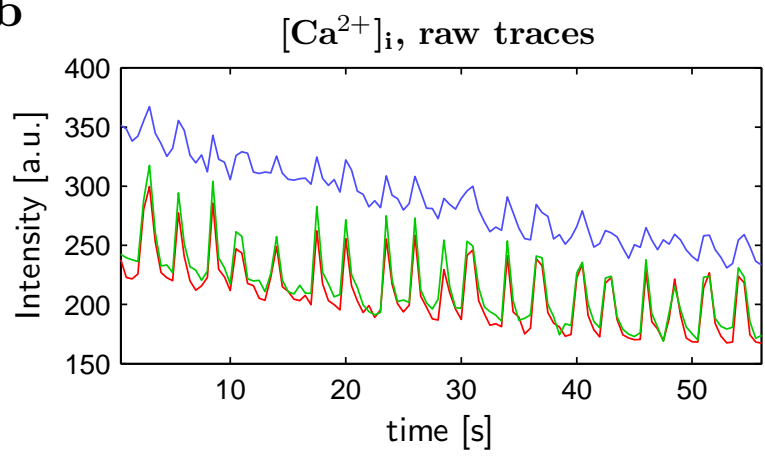

C

$\left[\mathrm{Ca}^{2+}\right]_{\mathrm{i}}$, bleach corrected and normalized

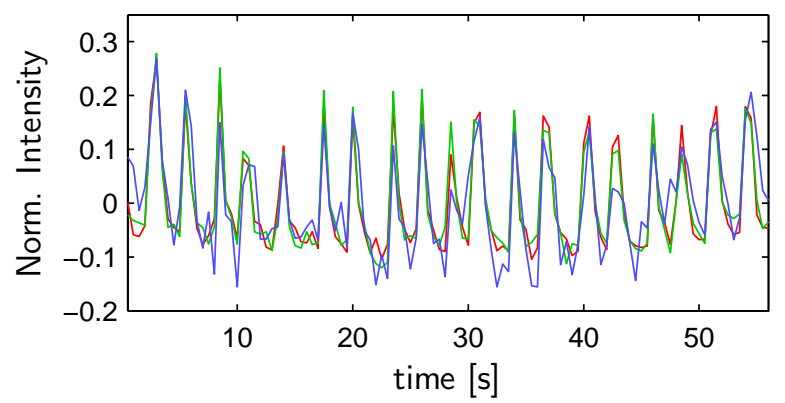

Figure 3.9: Imaging of spontaneous activity in the Xenopus laevis olfactory bulb. (a) A fluorescence image of the Xenopus olfactory bulb stained with $\mathrm{Ca}^{2+}$ indicator Fluo-4 (mean projection over time). The processes of the neurons are hardly visible due to a lack of contrast. Three regions are indicated by colored rectangles (arrowheads). Scale bar, $10 \mu \mathrm{m}$. (b) The fluctuations of the $\left[\mathrm{Ca}^{2+}\right]_{\mathrm{i}}$-dependent intensities of the three regions indicated in (a) appear very similar, despite different amplitudes. (c) The traces become virtually identical after correcting them for bleaching and normalization.

of the membrane permeable $\mathrm{Ca}^{2+}$ indicator Fluo-4/AM (Fig. 3.9). The high frame rates and the fast piezo actuator (3.1.3) of the LIM allows to image a significant fraction of the network by acquiring image stacks of about $20 z$-planes within $500 \mathrm{~ms}$. Within time series of such image stacks, individual pixels carry time-dependent signals. These reflect the fluctuations of the intracellular calcium concentration $\left[\mathrm{Ca}^{2+}\right]_{\mathrm{i}}$ of the imaged structures. In some cases, very distant pixels that do not show any relation or connection in the fluorescent intensity images (Fig. 3.9a) can be found to exhibit very similar temporal fluctuations (Fig. 3.9b). After correcting the traces for 
bleaching and for the different absolute intensities, the traces of these pixels become virtually identical (Fig. 3.9b), suggesting a functional or morphological relation between the underlying structures. This observation motivates the idea to exploit the time patterns of the $\left[\mathrm{Ca}^{2+}\right]_{i}$ fluctuations to visualize functionally related structures in the network, i.e. structures exhibiting similar $\left[\mathrm{Ca}^{2+}\right]_{\mathrm{i}}$ dynamics.

To visualize the spatial distribution of a particular time trace, 'activity crosscorrelation maps' were constructed. In this map, the brightness of each pixel encodes the cross-correlation value of the pixel's signal to a predetermined reference signal (e.g. the $\left[\mathrm{Ca}^{2+}\right]_{\mathrm{i}}$ signal of a particular soma or olfactory glomerulus). The crosscorrelation value of two traces is an indicator for their similarity, with highly similar traces producing a correlation value equal or close to one (Fig. 3.10b, top), and unrelated traces yielding a correlation value around zero (Fig. 3.10b, bottom). These correlation maps mostly showed a brightly labeled structure from which the reference signal was taken (Fig. 3.10c) on a relatively dark background. This reflects the fact that the spontaneous $\mathrm{Ca}^{2+}$ activities of most cells were uncorrelated (Chen et al., submitted). Interestingly, the correlation maps also revealed structures resembling neuronal processes (Fig. 3.10c), not seen in the raw fluorescence image (Fig. 3.10a).

\subsubsection{Combining ACI with fast time lapsed 3D imaging}

A two-dimensional correlation map as displayed in Figure 3.10c can be created with a conventional confocal or even widefield microscope. However, such a map mainly reveals neuronal processes close to the 'reference structure'. The LIM provides the possibility to virtually simultaneously acquire $\left[\mathrm{Ca}^{2+}\right]_{\mathrm{i}}$ signals from a number of different $z$-positions. It is thus possible to clarify whether the distal processes leave the optical slice or whether the correlations of the $\left[\mathrm{Ca}^{2+}\right]_{i}$ signals decrease along the processes. To this end, correlation maps to the same reference trace were calculated for all optical slices of the image stacks. Figure 3.11 shows for four (out of 18) slices the mean projection over time of the intensity images (a) as well as the corresponding correlation maps (b). It is obvious from these maps that temporally correlated structures can be found across the complete observed volume, with the neuronal processes meandering in and out of a given image plane. Each map by 
a

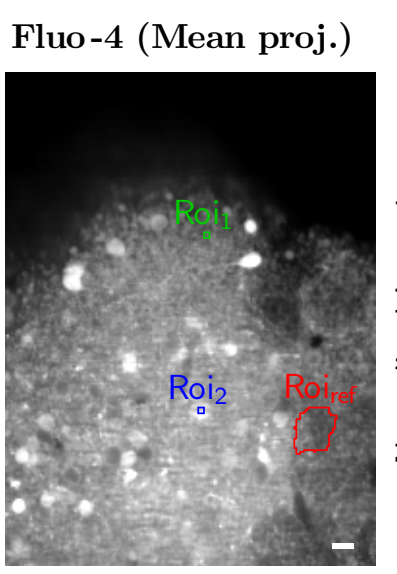

b

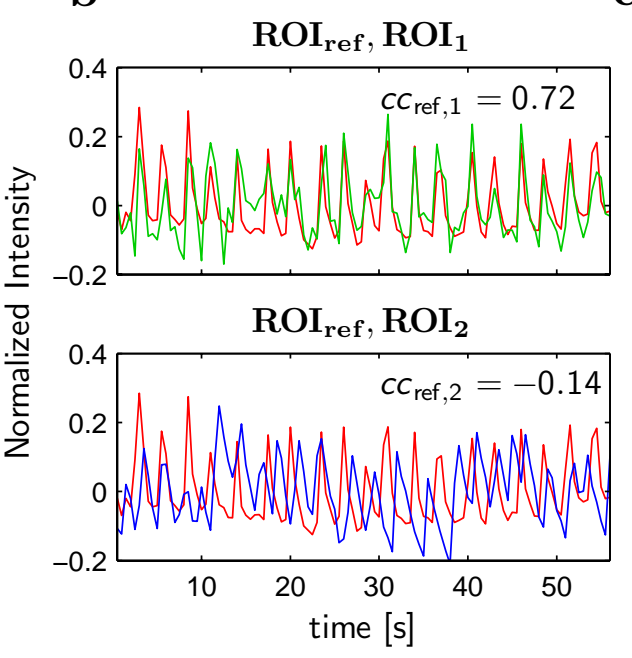

C Correlation Map

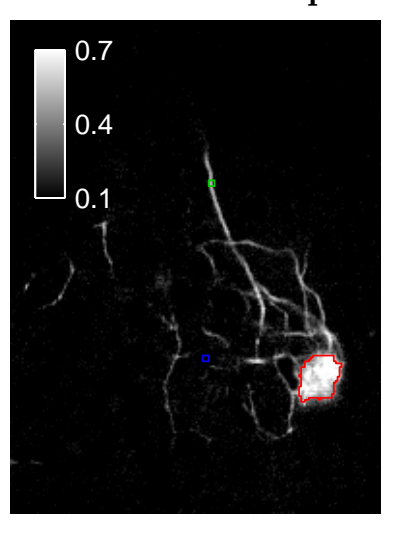

Figure 3.10: Principle of activity correlation imaging (ACI). ACI exploits the specificity of temporal fluctuations of the fluroescent signals to generate contrast. (a) Mean projection over time of fluorescent images of the Xenopus olfactory bulb. Indicated is a reference region $\mathrm{ROI}_{\mathrm{ref}}$ placed on an olfactory glomerulus (red) and two further regions, $\mathrm{ROI}_{1}$ (green) and $\mathrm{ROI}_{2}$ (blue). Scale bar, $10 \mu \mathrm{m}$ (applies also to (c)). (b) Top: The $\left[\mathrm{Ca}^{2+}\right]_{\mathrm{i}}$ fluctuations of $\mathrm{ROI}_{1}$ (green trace) and of $\mathrm{ROI}_{\text {ref }}$ (red trace) are very similar, yielding a cross-correlation value $c c_{\text {ref, }, 1}$ close to one. Bottom: In contrast, the $\left[\mathrm{Ca}^{2+}\right]_{\mathrm{i}}$ fluctuations of $\mathrm{ROI}_{\mathrm{ref}}$ and $\mathrm{ROI}_{2}$ (blue trace) are very different, resulting in a cross-correlation value $c c_{\mathrm{ref}, 2}$ close to zero. (c) For each pixel of the image frame, the crosscorrelation value between its own $\left[\mathrm{Ca}^{2+}\right]_{i}$ time course and the $\left[\mathrm{Ca}^{2+}\right]_{i}$ time course of the reference region was calculated. These values create a map that indicates which positions exhibit a time course similar to the reference region. This similarity in temporal behavior suggests a functional and / or morphological relationship between the underlying structures.

itself only shows some parts of the neuronal structures, their overall connectivity is revealed by a maximum z-projection (Fig. 3.11c). Alternatively, the spatial arrangement of the neuronal processes can be reconstructed into a three-dimensional model from the stack of correlation maps (not shown). Although such a map can contain a number of neurons, the 'labeling' is sparse enough to identify the neuronal processes and assign them to their respective somata. As long as the dendrites do not leave the observation volume, the connectivities between somata and glomerulus can be established unambiguously. This procedure of contrast generation is called 
a

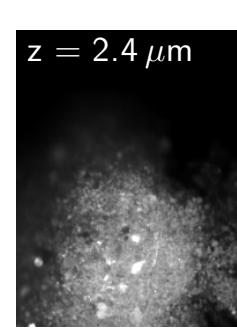

b

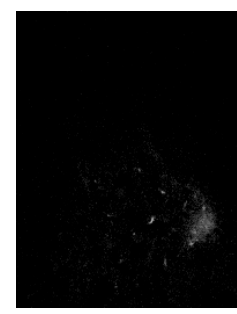

Fluo-4

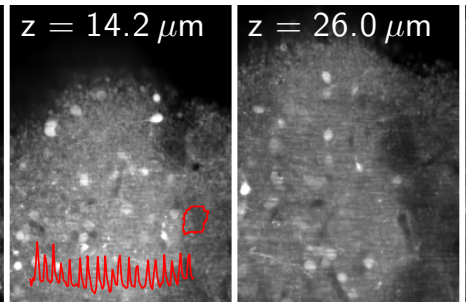

Correlation Maps
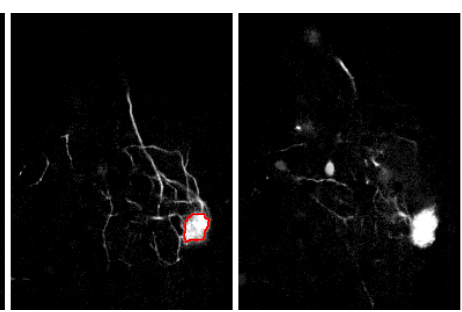

c

Correlation Map (Maximum Proj.)
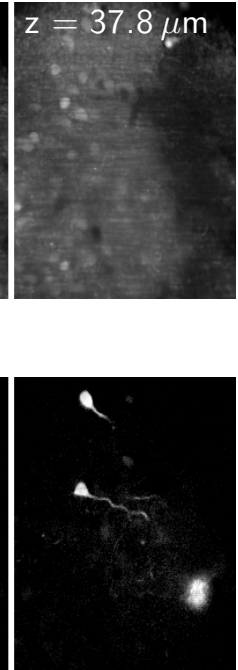

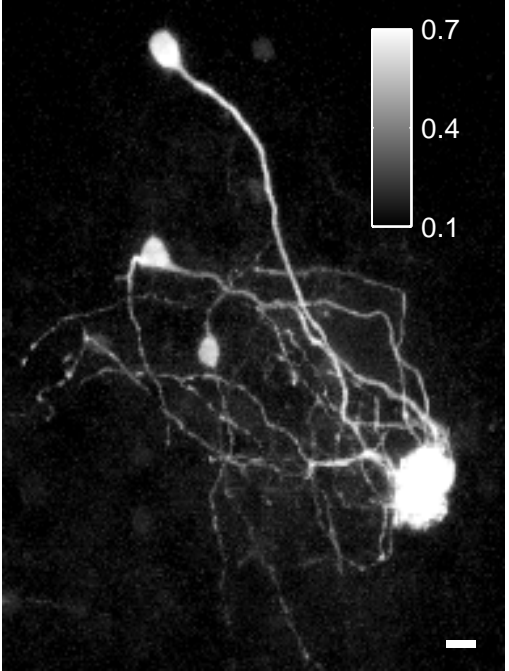

Figure 3.11: Activity correlation imaging using fast 3D image acquisition. Correlation maps can be calculated across all $z$-positions of the image stack, yielding a complete $3 \mathrm{D}$ reconstruction of the correlated structures. (a) Fluorescent images from four different $z$-positions as indicated. The reference region is indicated in red, its $\left[\mathrm{Ca}^{2+}\right]_{\mathrm{i}}$ time pattern is superimposed. (b) Correlation maps of the $z$ planes shown in (a), all of them being calculated with respect to the trace indicated in (a). (c) A maximum projection of all 18 planes of the experiment reveals the connectivity of the correlated structures, including the (reference) glomerulus, several somata and their dendrites. Due to the sparseness of the functional labeling, the processes are clearly identifiable and the connectivity between somata and glomerulus is obvious. The colorbar indicates the assignment of gray levels to correlation values. Scale bar, $10 \mu \mathrm{m}$.

'activity correlation imaging' (ACI). ACI can thus visualize processes and guide the measurement of signals from a neuron's processes even when they are located far away from the soma and not visible in the raw fluorescence image.

As individual neurons show specific activity patterns, it is possible to obtain many correlation maps using reference signals from different regions of interest (ROIs). Figure 3.12 shows six correlation maps as maximum z-projections as in Figure 3.10c with the respective $\left[\mathrm{Ca}^{2+}\right]_{\mathrm{i}}$ time traces superimposed. Many more of these maps can be created from a given data set and by assigning different colors to the resulting maps, it is possible to visualize the neuronal circuits with a markedly enhanced 
Correlation maps for different reference traces
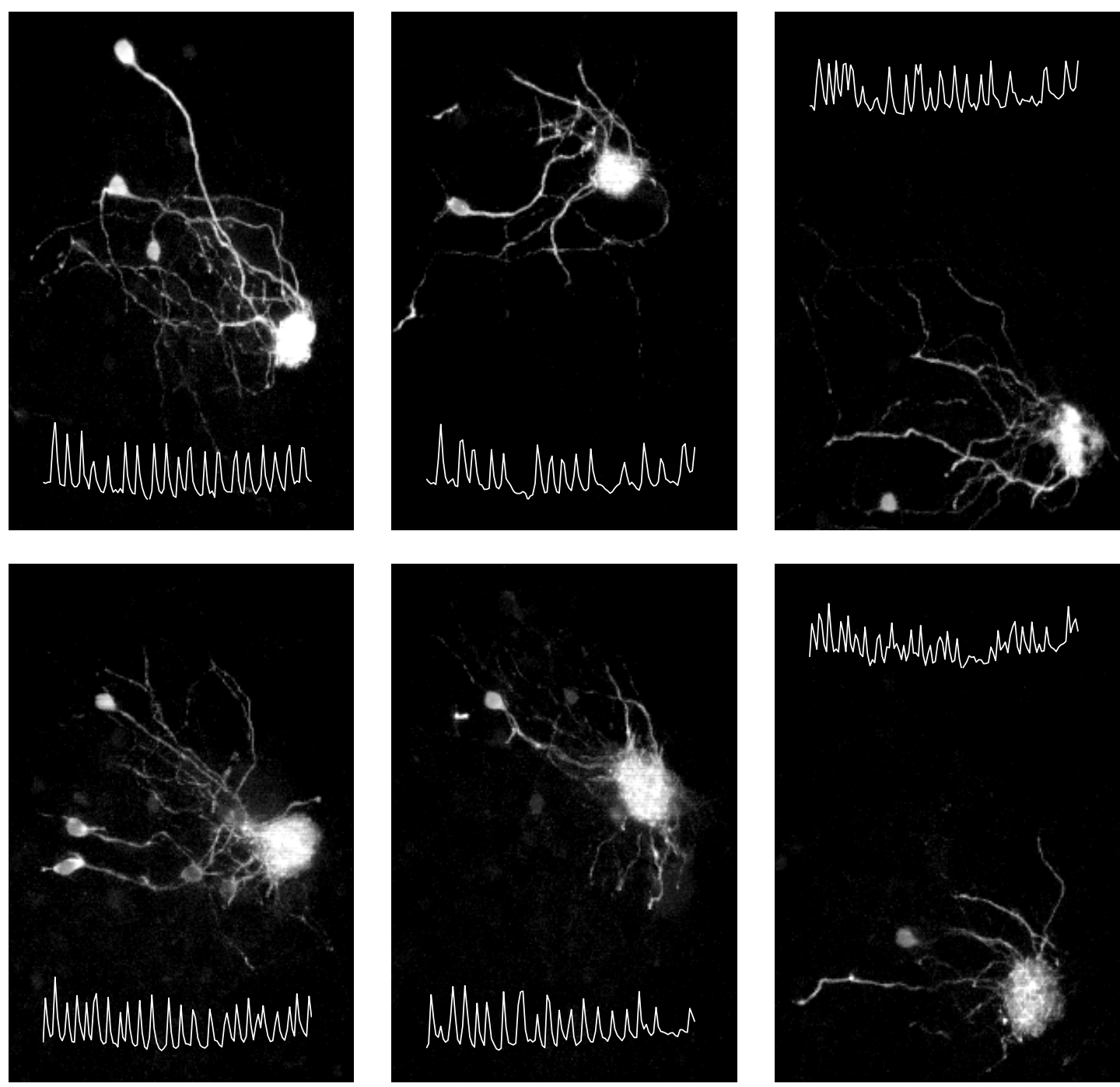

Figure 3.12: Using reference traces from different regions allows to create numerous correlation maps within the same experiment. Shown are the maximum $z$-projections of correlation maps that were created using the respective superimposed time traces. The colormap and the field of view is identical for all maps.

contrast. Figure 3.13a shows the time traces of 190 ROIs. Each of these traces can be used to create a correlation map. Assigning each of these maps a distinct color allows an almost complete visualization of many neurons and their processes (Fig. 3.13c $c_{1}$ ). Using different color schemes (Fig. 3.13b), it is possible to create 
a

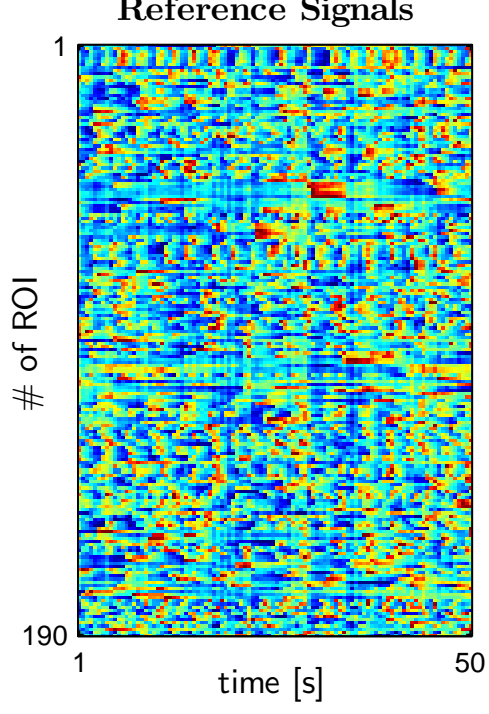

$\mathbf{c}_{2}$

LUT 2

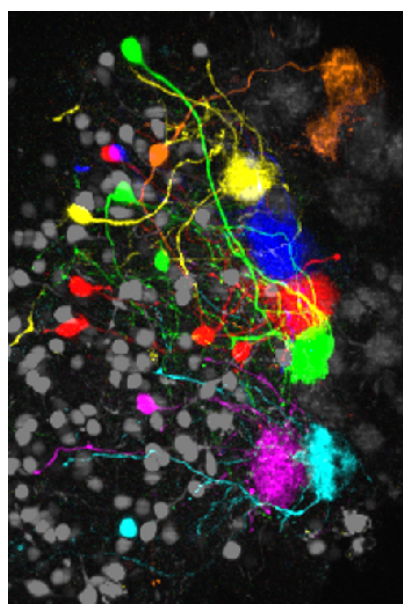

b

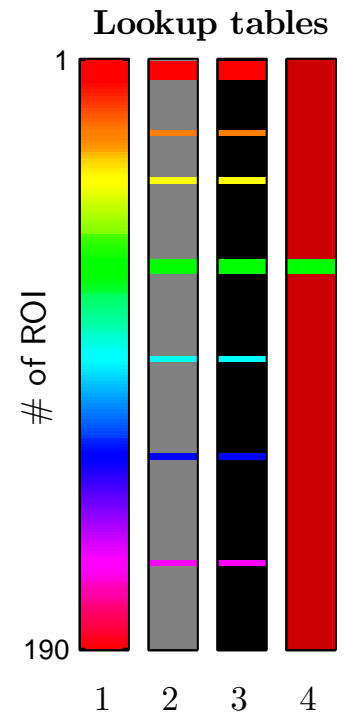

$\mathbf{c}_{3}$ $\mathbf{c}_{1}$ LUT 1

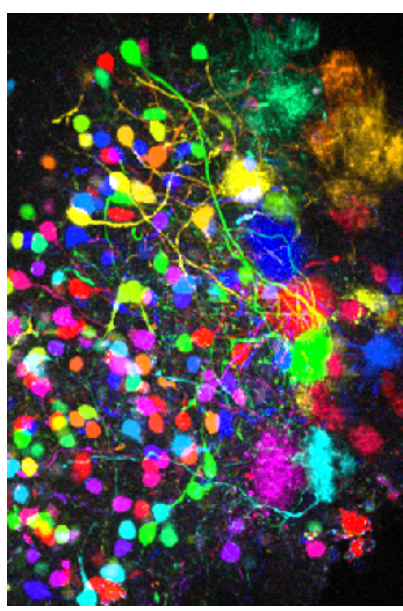

$\mathbf{c}_{4}$

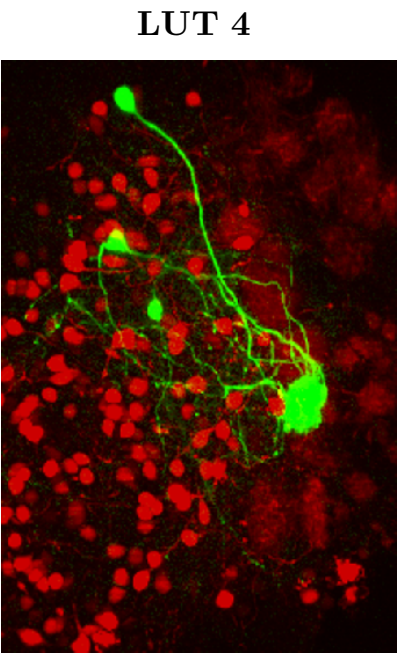

Figure 3.13: The numerous correlation maps can be combined into a multi-color display. (a) $\left[\mathrm{Ca}^{2+}\right]_{\mathrm{i}}$ signals of 190 different ROIs, arranged as a matrix with time in $x$ - and ROI index in $y$ direction. Color code of the time traces, dark blue to light red for low to high $\left[\mathrm{Ca}^{2+}\right]_{\mathrm{i}}$, respectively. (b) Four different color lookup tables chosen to highlight different features of the network. (c) Combining the correlation maps of different ROIs using the corresponding color lookup tables in (b). In these maps, the hue of each pixel is determined by the hue of the ROI that most correlates to the pixel's signal. The intensity was determined by the degree of correlation. The maps represent a multi-color visualizations of the complete network $\left(c_{1}\right)$, highlighting multiple $\left(c_{2}, c_{3}\right)$ or a single $\left(c_{4}\right)$ ensemble of correlated neurons. 
various multi-color visualizations of the network, each emphasizing different aspects of the network (Fig. 3.13c $c_{1}-c_{4}$ ). For example, one can selectively highlight multiple (Fig. $3.13 c_{2}$ ) or a single (Fig. $3.13 c_{3}$ ) ensemble of neurons to visualize how these neurons are embedded in their surrounding circuitry.

It is noteworthy that the neuronal activity patterns of these units are obtained from the functional imaging data. Combining the activity time traces with the deduced structural information reveals network structure and function in an unprecedented clarity.

\subsubsection{ACI and single cell dye injection}

As this polychromatic 'staining' generated by ACI is the result of a cross-correlation algorithm, its interpretation is less obvious than for example a fluorophore staining. A number of control experiments were performed, to compare the correlation maps (acquired with $40 \times 0.8 \mathrm{~W}$ objective) with the neuronal morphology as revealed by dye injections (imaged with $40 \times 1.2 \mathrm{~W}$ objective). To this end a complete set of 3D correlation maps was generated online, and a particular neuron, selected from one of the maps, was filled with fluorescent tracer through a patch pipette. This experiment may also serve to demonstrate that this method can be used as an online-tool. Figure 3.14a shows the staining obtained after the pipette was detached from the cell, while Figure $3.14 \mathrm{~b}$ shows the correlation map of this neuron. The overlay in Figure 3.14c confirms that the structures emerging from the soma of the selected neuron in the correlation map are indeed processes of this cell. It was never observed, that structures in the correlation map branching off from the selected neuron were not visible in the injection image. Of course, the correlation maps revealed - by definition - additional somata and dendritic segments with highly correlated activity, whenever present.

Combined ACI and dye injection were performed in five cells. The comparison of the morphology of the investigated neurons resulted in the following numbers (dye injection / ACI): primary dendrites emerging from the soma and ending in a glomerulus: 6 / 6 (1 or 2 per cell); branching of dendrites (sub-branches leaving the observation volume in some cases): $7 / 9$ (1 - 3 per cell); further processes emerging 

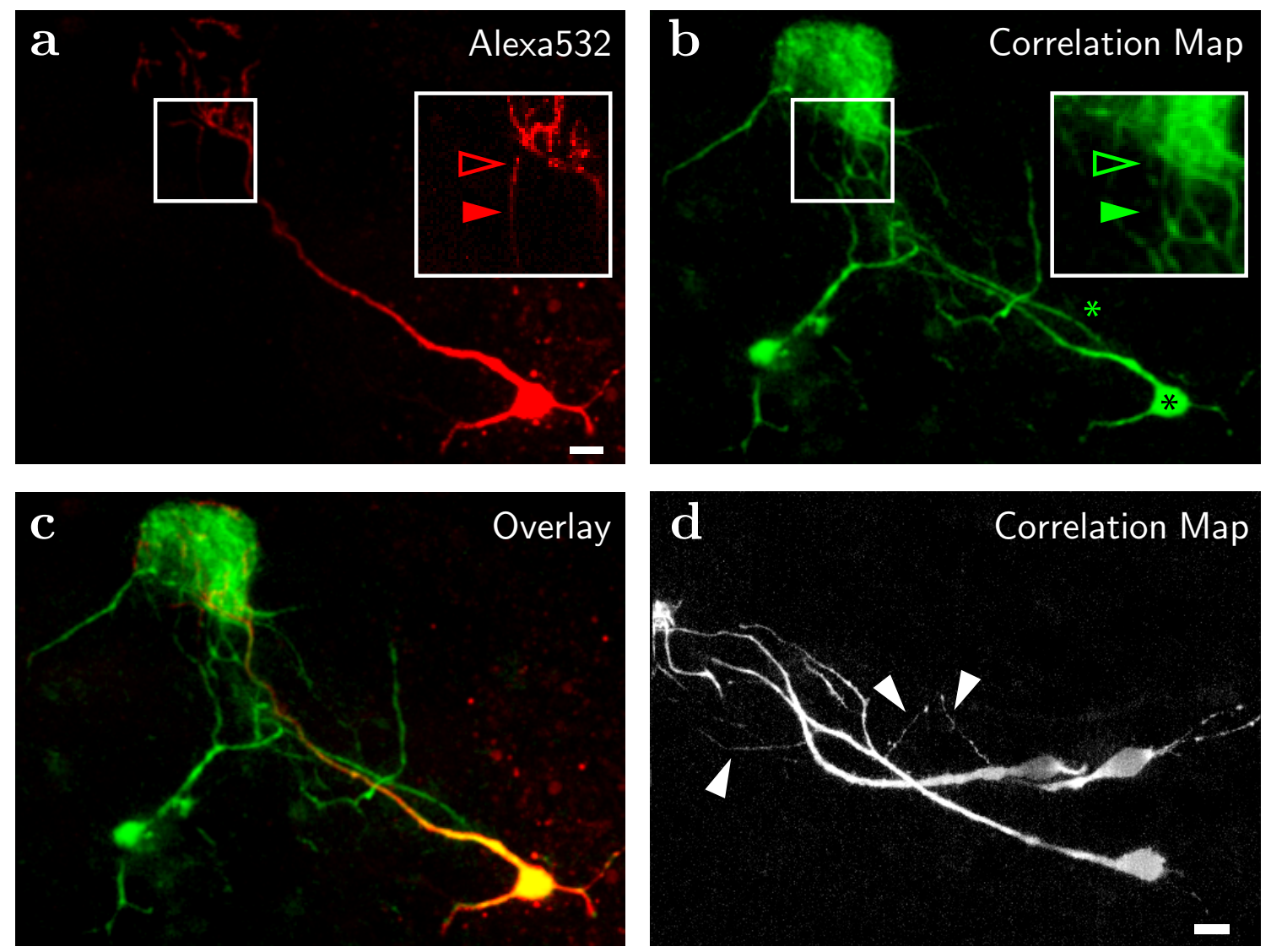

Figure 3.14: ACI and dye injection. (a) Intensity image after dye injection with a patch pipette (Alexa 532). The neuron's processes that are visible in the correlation map (b) are all present here. Some dendrites appear only very faint, indicating their small diameter (inset, empty and filled arrowheads). The inset is a projection of only three out of $24 z$-planes. Scale bar $10 \mu \mathrm{m}$. (b) From this correlation map, the soma was selected for dye injection (black star). The inset shows a magnification of the indicated part (same as in a), with the empty arrowhead pointing at a part of a dendrite that disappears into noise (filled arrowhead). The marked dendrite (green star) does not belong to the filled neurons and only seems to branch off of the primary dendrite due to the projection of the image stack. The image is slightly gauss-filtered (width 0.8 pixel) for improved display. (c) The overlay of the correlation map with the injection image confirms that the majority of the structures of the filled neuron is present in both images. (d) Correlation map acquired with a higher magnification objective. Thin secondary dendrites can be identified unambiguously (filled arrowheads). Scale bar, $10 \mu \mathrm{m}$.

from the soma (possibly axons): $3 / 5$ (0 - 2 per cell).

Occasionally, very thin structures that were observable in the injection-labeled 
image were missing in the correlation map (see insets of Fig. 3.14a and 3.14b, arrowheads). To address the question whether these thin processes were generally inaccessible to this technique, ACI was performed using a higher magnification objective $(63 \times 0.95 \mathrm{~W})$. As can be seen in Figure $3.14 \mathrm{~d}$, the correlation maps of these experiments clearly revealed very thin dendritic structures, proving that ACI can detect these structures as long as they exhibit correlated $\left[\mathrm{Ca}^{2+}\right]_{\mathrm{i}}$ signals.

\subsubsection{Discussion of ACI}

ACI can generate a virtually unlimited number of correlation maps in a single experiment, and coding each of them with a different color results in a multi-color visualization of the complete neuronal network.

Recently, multi-color visualization of neuronal networks has been realized using a transgenic approach (Livet et al., 2007). In the Brainbow transgenic mice, a differential expression of $2-4$ different fluorescent proteins is used to provide the contrast to distinguish neurons. Each neuron in Brainbow is thus coded by $2-4$ variables, each representing the expression level of a particular fluorescent protein. ACI takes a different and partly complementary approach. The neurons are stained with a single fluorescent dye, and the neurons' specific temporal activity waveforms are exploit as a contrast variable. In this way, each neuron is 'labeled' by an $N$ dimensional temporal activity waveform, with $N$ being the number of sample points in time. As the number of time points can be increased (limited only by bleaching), ACI provides a very high specificity to distinguish neurons. This is manifested by the fact that individual correlation maps typically reveal only one or few neurons and their processes with little unspecific labeling. A further advantage of obtaining contrast in the 'time domain' is that this leaves the unused spectral channels available for other potential applications. For example, one might use fluorescent dyes of different colors to label the expression of certain proteins or molecules. This would make it possible to simultaneously map the expression of molecules, the expression of functional activities, and the morphology of neurons.

ACI develops its full potential when combined with a fast image acquisition system. Recently, a number of fast 3D imaging systems have been developed based 
on point scanning through a predefined trajectory (Göbel et al., 2007), AOD scanning (Reddy and Saggau, 2005; Vucinić and Sejnowski, 2007) or planar illumination (Holekamp et al., 2008). Combined with bolus loading of $\mathrm{Ca}^{2+}$ indicators, these systems allow the imaging of many neuronal somata distributed in 3D. To visualize dendritic structures, however, the labeling density must be reduced, e.g. by electroporation (Nagayama et al., 2007) or by single cell dye injections. But this intrinsically limits the number of neurons that can be imaged to at most a few percent of all neurons. In contrast, the ACI approach provides the simultaneous visualization of both the functional activities and the dendritic connections of virtually all active and dye-loaded neurons in the volume of interest.

We performed ACI in the OB taking its spontaneous activities as the basis. Spontaneous activity has been recorded by use of calcium indicator dyes in many parts of the brain, including the cortex (Ikegaya et al., 2004; Mao et al., 2001; Stosiek et al., 2003) and the hippocampus (Usami et al., 2008). In some cases, small numbers of neurons were found to be synchronized (Mao et al., 2001), similarly to the $\mathrm{M} / \mathrm{T}$ cells innervating the same glomerulus in the OB. Thus, with respect to the occurrence and properties of the spontaneous activity, our model system appears to be representative for a large number of networks in the brain. Obviously, ACI could also be based on evoked activity, as long as the subset of activated neurons is either sparse or inhomogeneous with respect to the temporal response patterns.

It should be noted that the correlation map reveals the parts of an image that exhibit changes in fluorescence similar to the selected ROI. This might be a group of neurons, an entire neuron, or a sub-compartment of a cell. In cell types where there is a strong action potential back-propagation into the dendritic tree (e.g. mitral cells (Bischofberger and Jonas, 1997; Charpak et al., 2001); pyramidal neurons or substantia nigra dopaminergic neurons (Stuart et al., 1997)), the correlation map is likely to reveal a substantial part of the cell, as we have shown here for the case of mitral cells. ACI may also be useful to study cell types where action potential back-propagation is relatively weak (e.g., Purkinje neurons (Stuart et al., 1997)). In these cases one might use the signals from different dendritic segments as reference signals to construct complementary correlation maps. This might reveal the whole 
cell's morphology but also the extent of functional sub-compartmentalization within a single neuron and thus help to elucidate how these neurons integrate and process information.

While compartmentalization of fluorescent dye in organelles was not an issue for the performance of ACI in our preparation, it might potentially affect the results of the ACI algorithm. It might prove beneficial to place the reference ROIs in organelle-free parts of the cell (e.g. periphery of the soma or olfactory glomerulus) and to minimize compartmentalization by optimizing the experimental parameters, such as the incubation temperature and the choice of the calcium indicator dye (for a review, see Takahashi et al. (1999)).

An additional strength of the method is that the computation of correlation maps can be done very fast. Using Matlab on a common PC, it is possible to calculate 190 correlation maps $(512 \times 256 \times 18$ pixels each, 112 time points, see Fig. 3.13$)$ in $30 \mathrm{~s}$, which is within the time for acquiring the actual data. The ability to map function and structure of neuronal populations online opens up a number of intriguing applications. It allows selecting specific cells (Fig. 3.14) or cell pairs (Chen et al., submitted) for targeted electrophysiological recordings. Further applications might include selecting neurons with certain functional and/or projection profiles for targeted ablation or stimulation. This way it will be possible to investigate how these changes affect the network function.

Taken together, ACI opens up novel ways for investigating and manipulating neuronal networks with an unprecedented specificity. 


\subsection{Olfactory Coding with Population Onset Times}

Information about an olfactory stimulus are contained in the spike patterns of mitral/tufted $(\mathrm{M} / \mathrm{T})$ cells at the level of the olfactory bulb (OB). Even for very simple stimuli (e.g. mono-molecular odorants) this information is distributed over numerous cells. The relative timing of the response patterns of the different neurons is assumed to be important for the coding of an odor (Friedrich and Laurent, 2001; Kauer, 1991; Laurent, 1999; Laurent et al., 2001). Findings in other sensory systems and behavioral studies in the rodent and human olfactory system motivate a detailed investigation of the early phase of the odor-evoked $\mathrm{M} / \mathrm{T}$ cell response patterns (Abraham et al., 2004; Gollisch and Meister, 2008; Kiesecker et al., 1996; Laing, 1986; Slotnick, 2007a; Verhagen et al., 2007).

Different parameters of the early $\mathrm{M} / \mathrm{T}$ cell response patterns have been suggested to be important for olfactory coding: the time of the response onset, the frequency of the evoked spike train in a neuron, and the number of elicited action potentials (Bathellier et al., 2008; Margrie and Schaefer, 2003). The present study aims at investigating the time of the response onset (which is also referred to as response latency) for single neurons and particularly the relations between onset times across neurons in the OB. Unlike the response frequency and the number of action potentials, the onset times of single $\mathrm{M} / \mathrm{T}$ cells have been suggested to be odor and concentration dependent across species (insects (Wilson et al., 2004), fish (Yaksi et al., 2007) and mammals (Spors et al., 2006)). Additionally, the timing of the first spike has been implicated in coding strategies in various sensory systems (Gollisch and Meister, 2008; Johansson and Birznieks, 2004; Petersen et al., 2001). This makes the pattern of response latencies a likely candidate for a general (species independent) odor specific aspect of the $\mathrm{M} / \mathrm{T}$ cell response pattern. Additionally, response times provide an immediate and thus fast measure, and efficient strategies for decoding the order of response onset patterns have been proposed (Gütig and Sompolinsky, 2006). The analysis of onset times was thus extended to an investigation of the order in which ensembles of $\mathrm{M} / \mathrm{T}$ cells start responding to a given stimulus. 
An investigation of the patterns of response onsets requires the simultaneous observation of the activity of a large number of neurons with a temporal resolution that allows to distinguish the onset times of many neurons within a short time interval. The LIM provides fast confocal imaging, and functional dyes can be used to visualize neuronal activity of cell populations. Tissue slices of the OB of Xenopus laevis tadpoles were thus stained with the $\mathrm{Ca}^{2+}$ sensitive fluorescent dye Fluo-4/AM. The onset time of a neuron is defined by the time of the first odor-evoked spike. Since the $\mathrm{Ca}^{2+}$ dependent fluorescence signal is known to reflect the electrical activity of $\mathrm{M} / \mathrm{T}$ cells in Xenopus (Lin et al., 2007), the $\mathrm{Ca}^{2+}$ trace can be expected to show a sharp rising phase at the beginning of the response, which facilitates the detection of the onset time. Amino acids were used as stimuli, as they have been shown to be behaviorally relevant odors in tadpoles (Kiseleva, 1983, 1995) and other amphibians (Ferrer and Zimmer, 2007).

\subsubsection{Measuring odor-evoked responses with high temporal resolution}

The determination of the response onset times requires a high temporal resolution for the investigation of the onset of the odor-evoked response. Additionally, the acquisition of pre- and post-stimulus time periods are necessary to evaluate the level of spontaneous activity and the late phase of the response. For these phases of the image acquisition, a lower temporal resolution is however sufficient. The custom built LIM offers the possibility of dynamic scan protocols. This allows to change the temporal resolution within a single acquisition period, and thereby to restrict high frame rates to a 'time interval of interest'. In this way bleaching of the fluorescent dye is minimized, and thus a large number of stimulus applications can be carried out on a single slice. In the following, an (odor) application refers to the image sequence acquired during a single odorant stimulation, while an experiment refers to all applications carried out on a single olfactory bulb slice. Different stimuli were applied in a randomized order, with a minimum inter-stimulus interval of $1.5 \mathrm{~min}$. Application of ringer (bath solution) was used as a negative control.

Figure 3.15a shows a typical scan protocol used for most experiments in this section. The baseline of the intracellular calcium level is recorded for $5 \mathrm{~s}$ at $2 \mathrm{~Hz}$, 
$\mathbf{a}$

Scan Mirror Position ( $y$-position)
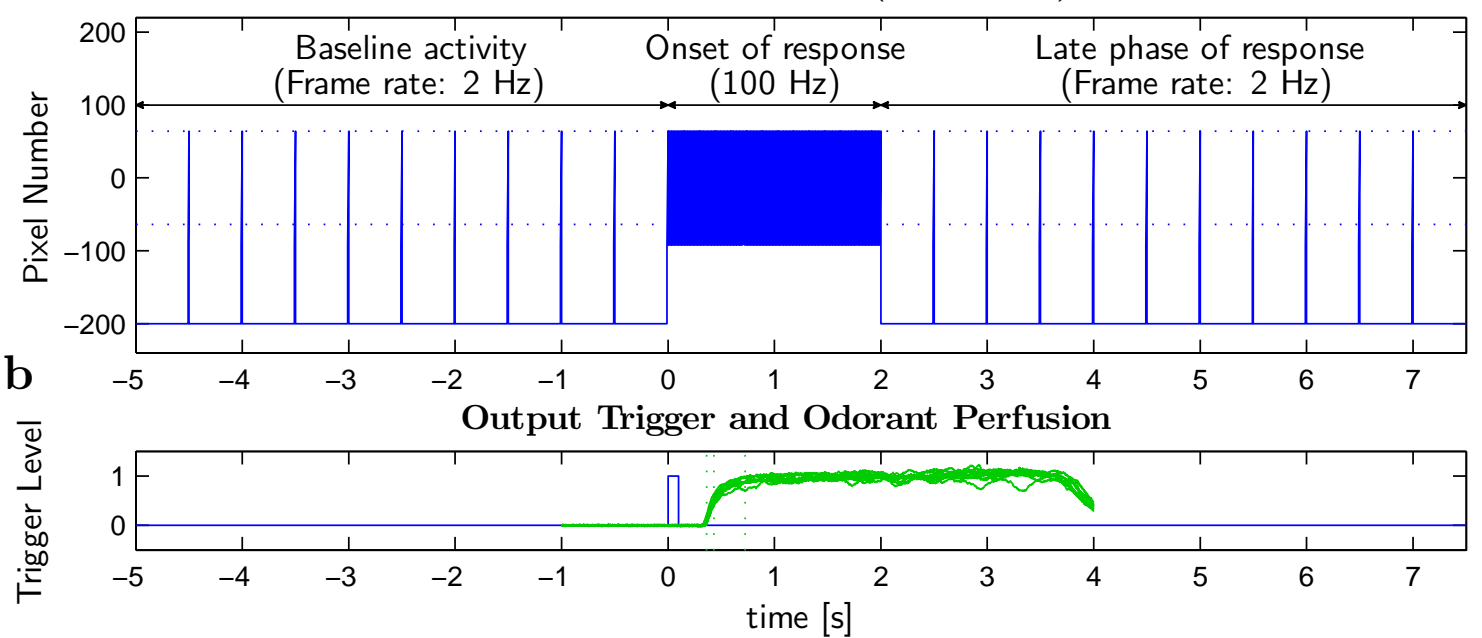

C

Fluorescence Images
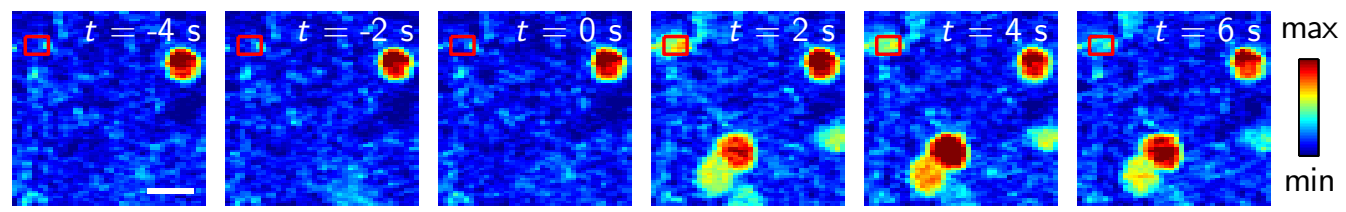

Fluorescence Intensity

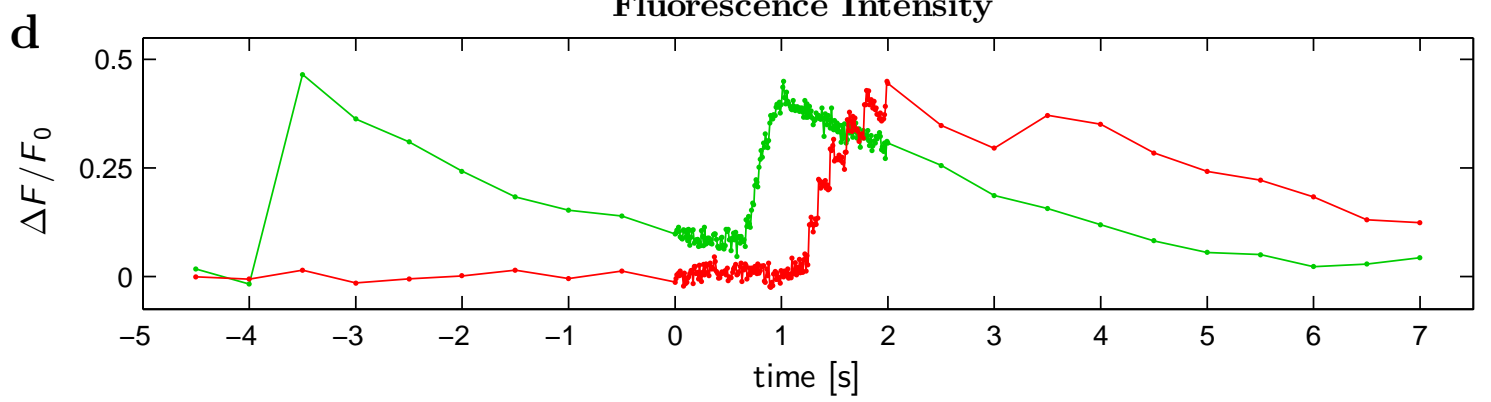

Figure 3.15: Schematics of scan protocol for odor stimulation experiments. (a) The onset of the response is imaged with a high temporal resolution $(100 \mathrm{~Hz})$. To minimize photo-bleaching, the baseline and the late part of the response are acquired with a lower resolution (2 $\mathrm{Hz})$. The scan mirror returns to the 'light-off' position in between frames. The dotted horizontal lines indicates the imaged area, the scanned area (blue rectangle) is slightly larger due to the additional "preacquisition lines' (see 3.1.3). One frame consists of 128 lines in $y$-direction that cover a length corresponding to 256 pixels in $x$-direction, resulting in rectangular pixels. (b) The odor delivery is initiated by a trigger from the control PC (blue curve). The normalized time course of ten applications, visualized by adding fluorescent dye to the solution, are shown in green. Vertical green lines indicate the averaged times of $10 \%, 50 \%$ and $90 \%$ of the maximum concentration (0.36 s, $0.42 \mathrm{~s}$ and $0.72 \mathrm{~s}$ after trigger onset, respectively). (c) The scanning procedure results in a sequence of fluorescence images. Shown are six images acquired at the indicated time points. The red outline indicates the position of a neuronal soma. Scale bar, $10 \mu \mathrm{m}$. (d) By averaging the pixel over a soma, the temporal pattern of $\mathrm{Ca}^{2+}$ dependent fluorescence intensities can be extracted from the image series, shown as $\Delta F / F_{0}$ values. The red curve shows the time trace of the region indicated in (c), the green curve shows the trace of a spontaneously active neuron outside the field of view. During the time of fast scanning, the neuronal activity is captured with great detail. 
followed by a high speed acquisition at $100 \mathrm{~Hz}$ for $2 \mathrm{~s}$, and finally another $5 \mathrm{~s}$ acquisition at $2 \mathrm{~Hz}$. The odor application is triggered to the central acquisition phase with the arrival of the odor being delayed by few hundred milliseconds (see Fig. 3.15b). This delay might vary from slice to slice due to the positioning of the perfusion cannula relative to the mucosa, but it is highly reproducible for repeated applications in one slice (Fig. 3.15b). The result of this scanning scheme is a series of fluorescence images with varying inter-image time intervals (representative examples are shown in Fig. 3.15c). By outlining the area of a neuronal soma with a region of interest (ROI, red rectangle in Fig. 3.15c) and spatially averaging the fluorescence intensities of the pixels included in this area, the relative $\left[\mathrm{Ca}^{2+}\right]_{\mathrm{i}}$ time course of this neuron can be extracted from this image series. Typically this time course is displayed after correcting for the baseline $\left[\mathrm{Ca}^{2+}\right]_{\mathrm{i}}$ level as $\Delta F / F_{0}=\left(F(t)-F_{0}\right) / F_{0}$ value, with $F(t)$ being the fluorescence measured at time $t$, and $F_{0}$ being the fluorescence prior stimulation (temporal average). The red curve in Figure 3.15d represents the fluorescence time course of the stimulated neuron outlined in Figure 3.15c. The green trace (extracted from a neuron outside the field of view in Fig. 3.15c) displays a spontaneously active neuron. Without the pre-stimulus image acquisition, the activity around $t=5.6 \mathrm{~s}$ could be interpreted as an odor-induced response, which is unlikely, given the similar $\left[\mathrm{Ca}^{2+}\right]_{\mathrm{i}}$ transient prior stimulus application (around $t=1 \mathrm{~s})$. Levels of spontaneous activity were evaluated based on the time traces of all applications. Traces with elevated levels of spontaneous activity were not included in the analysis.

\subsubsection{Determination of the response onset time}

The following experiments concern the reproducibility and specificity of the onset time of the odor induced response, both for single neurons and for neuronal ensembles. In previous studies, the response onset was determined by fitting a sigmoidal function to the $\mathrm{Ca}^{2+}$ dependent fluorescent time course (Spors and Grinvald, 2002; Spors et al., 2006). While this approach yields an estimate of the response begin, it is unlikely to capture the onset time with a sufficient precision, as it is based on the global shape of the response pattern. Since the current study aims at exploring the 

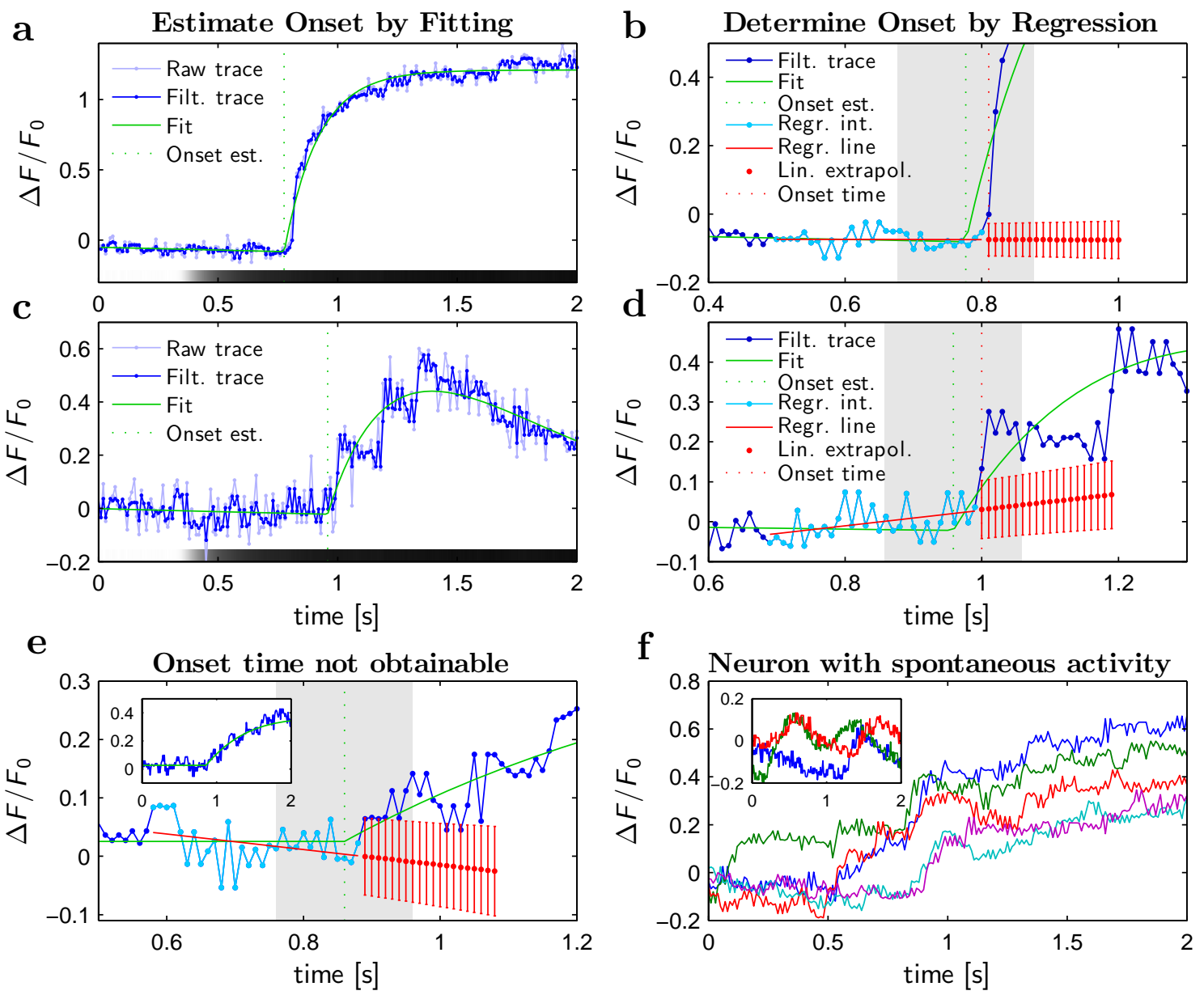

Figure 3.16: The onset of the response is determined in three steps, demonstrated using the responses of two neurons (first and second row). Horizontal bars at the bottom of the graphs in (a) and (b) indicate stimulus application (gray values correspond to averaged values from Fig. 3.15b). $(\mathbf{a}, \mathbf{c})$ First, the raw traces (light blue) are smoothed using an edge-preserving filter (dark blue). Then, a function is fitted to the data (fitted curve: green solid line, estimate for onset: green broken line; see text for details) to determine an estimate of the response onset. (b,d) The precise onset is determined by a local analysis restricted to the interval of $\pm 100 \mathrm{~ms}$ around the estimated value (light gray area around broken green line). From the linear regression (solid red line) of the data points within a moving window (cyan line), prediction intervals are calculated (red errorbars). The window is moved from early towards later times, and the onset is defined as the first out of 20 consecutive time points that are outside the prediction intervals (broken red line). (e) For some traces, the onset cannot be determined due to an insufficient signal-to-noise ratio. These curves were not used in the further analysis. The displayed prediction intervals were calculated at the presumed onset. The inset shows unambiguously that the neuron was active during the observation interval. (f) Some neurons show high levels of spontaneous activity. Since this is a potential error source, these neurons could not be included in the analysis. Shown are responses of a neuron to repeated applications of a stimulus. The reaction to the stimulus was superimposed by spontaneous activity (compare to control measurements, inset), which makes a reliable determination of the response onset impossible. 
latencies of a large number of neurons within a limited time interval, it is crucial to use a precise method that works equally well for different shapes of evoked $\mathrm{Ca}^{2+}$ response patterns. A novel method for a reliable determination of the response onset time was thus devised. The determination of the response onset consists of three steps (see Fig. 3.16 for two exemplary $\mathrm{Ca}^{2+}$ traces):

1. The trace is smoothed using the edge preserving Kuwahara filter (Kuwahara et al., 1976) with a width of $30 \mathrm{~ms}$ (three time points). The use of an edgepreserving filter ensures a suppression of noise without 'blurring' the sharp response onset (Fig. 3.16a and c, light and dark blue curves show raw and filtered signal, respectively).

2. A piecewise defined function $f_{\text {fit }}$, consisting of a linear 'baseline' in the first part followed by a double-exponential function containing both a rising and a declining term, is fitted to the trace (see Fig. 3.16a and c, green solid line):

$$
f_{\text {fit }}(t)= \begin{cases}a_{\text {lin }} \cdot t+f_{0} & , t<t_{\text {on }}^{\text {fit }}, \\ a_{\text {lin }} \cdot t_{\text {on }}^{\mathrm{fit}}+f_{0}+a_{\text {exp }} \cdot \mathrm{e}^{-\left(t-t_{\text {on }}^{\text {fit }}\right) / \tau_{\text {down }}} \cdot\left(1-\mathrm{e}^{-\left(t-t_{\text {on }}^{\mathrm{fit}}\right) / \tau_{\text {up }}}\right), t \geq t_{\text {on }}^{\mathrm{fit}}\end{cases}
$$

The fast rising and slowly declining exponentials account for the response onset and the subsequent $\mathrm{Ca}^{2+}$ outflux, respectively. The following values are fitting parameters: The coefficients $a_{\operatorname{lin}}$ and $a_{\exp }$, the value $f_{0}=f(t=0)$, the time constants $\tau_{\text {up }}$ and $\tau_{\text {down }}$ of the exponential terms and the onset estimate $t_{\mathrm{on}}^{\mathrm{fit}}$ that separates the two sections of this function (Fig. 3.16a and b, green broken vertical line).

3. To determine the precise onset time, a local regression analysis is performed in a time window $\pm 100 \mathrm{~ms}$ around the onset estimate $t_{\mathrm{on}}^{\mathrm{fit}}$. The onset is characterized by a significant deviation from the linear baseline. To determine this time point, the linear regression $f_{\text {regr }}$ (red solid line) over a time window of $300 \mathrm{~ms}$ (cyan data points) is used to calculate linear prediction intervals for later time points (red error bars):

$$
f_{\text {regr }}\left(t_{n}\right)=a_{\text {regr }} \cdot t_{n}+b+\epsilon,
$$


assuming a normal distribution of the residuals $\epsilon=N\left(0, \sigma^{2}\right)$. Using $M$ time points for the regression, the prediction interval for the $(M+1)^{\text {st }}$ value to be a continuation of the linear trend is given by (Beichelt and Montgomery, 2003)

$$
\begin{aligned}
& {\left[f_{\text {regr }}\left(t_{M+1}\right)-t_{M-2, \alpha / 2} s \sqrt{1+\frac{1}{M}+\frac{\left(t_{M+1}-\bar{t}\right)^{2}}{s_{t t}}},\right.} \\
& \left.f_{\text {regr }}\left(t_{M+1}\right)+t_{M-2, \alpha / 2} s \sqrt{1+\frac{1}{M}+\frac{\left(t_{M+1}-\bar{t}\right)^{2}}{s_{t t}}}\right],
\end{aligned}
$$

where $f_{\text {regr }}\left(t_{M+1}\right)=a_{\text {regr }} \cdot t_{M+1}+b$ is the predicted value at time point $t_{M+1}$, $s^{2}=\frac{1}{M-2} \sum_{n=1}^{M}\left(f_{\text {meas }}\left(t_{n}\right)-f_{\text {regr }}\left(t_{n}\right)\right)^{2}$ is the empirical residual variance of $f_{\text {meas }}\left(t_{n}\right), s_{t t}=(M-1) s_{t}^{2}=\sum_{n=1}^{M}\left(t_{n}-\bar{t}\right)^{2}$ is the variance of the time data and $t_{M-2, \alpha / 2}$ is the $\left(1-\frac{\alpha}{2}\right)$ - quantile of the $t$-distribution with $M-2$ degrees of freedom ('-2' for the regression parameters $a_{\text {regr }}$ and $b$ ). The time window is moved from early towards later times (starting from $t_{M+1}=t_{\mathrm{on}}^{\mathrm{fit}}-100 \mathrm{~ms}$ ), until twenty consecutive data points are found outside of the prediction intervals. The analysis was found to be insensitive to the required number of time points, as long as it is sufficiently large to ensure that the deviation from the linear trend is significant. The first of these time points is defined as the odor onset (indicated by red vertical line in Fig. 3.16b and d).

While the algorithm performs well for most time traces (Fig. 3.16a-d), there are cases where, despite the smoothing, the signal-to-noise ratio is too small and the onset time is not accessible using the described algorithm (see example in Fig. 3.16e). These traces could not be included in the subsequent analysis. Furthermore, while the global fit (step 2 of the algorithm) is rather insensitive to noise and occasional spontaneous activity, elevated levels of spontaneous activity were a potential error source for the algorithm. Figure $3.16 \mathrm{f}$ shows an example of a neuron that displayed a superposition of spontaneous and odor-evoked activity. Neurons showing consistently high levels of spontaneous activity were not included in the analysis. The occasional occurrence of non-odor dependent activity remained however a potential source for erroneous onset times. 


\subsubsection{Reproducibility and concentration dependence of response onset times}

A typical experiment for the investigation of response onset patterns of $\mathrm{M} / \mathrm{T}$ cells is shown in Figure 3.17. The olfactory bulb tissue slice was stained with the $\mathrm{Ca}^{2+}$ indicator dye Fluo-4 (overview of bulb hemisphere in Fig. 3.17a), and the M/T cell layer is used for image acquisition (red outline in Fig. 3.17a and Fig. 3.17b). To facilitate the selection of responsive neurons, an 'autocorrelation map' was calculated (Fig. 3.17c). This map highlights the regions that show similar time courses upon repeated application of a stimulus (see Materials and Methods for details). Figure 3.17d shows exemplary the time courses of the four ROIs indicated in Figure $3.17 \mathrm{c}$ for four applications of the same stimulus (arginine, $25 \mu \mathrm{M}$ ). The responses are highly reproducible, including the time of response onset. In order to compare the onset times of the neurons during the individual applications, the same data are shown in Figure 3.17e sorted by application. The times of response onset, determined using the algorithm described above, are indicated by vertical lines. The onset order of the four cells is nearly identical for all applications, indicating a high reproducibility of the population onset pattern. Solely the onset order of cells 1 and 2 are exchanged in the last application. This motivates the investigation of the reproducibility and stimulus dependence of the response latencies of individual $\mathrm{M} / \mathrm{T}$ cells as well as of onset patterns of ensemble of $\mathrm{M} / \mathrm{T}$ cell.

First, the variability of response onset times was investigated from 247 cells in 14 slices, stimulated with a variety of stimuli (varying both in odor and concentration). Each stimulus was repeatedly applied (6 - 8 times), and the standard deviation of the response onset was calculated for each cell across all applications of a given stimulus. As not all cells responded to all applications, and as the response times could not be assessed for all traces, the standard deviations are based on a different numbers of values. Figure 3.18a shows the distribution of standard deviations, with different colors indicating different number of measurements from which the standard deviation was calculated. The distribution has a peak around $40 \mathrm{~ms}$, a median of $84 \mathrm{~ms}$ and few values larger than $200 \mathrm{~ms}$. In contrast, the standard deviation calculated over all recorded onset times is $288 \mathrm{~ms}$. To test whether the variability 
a

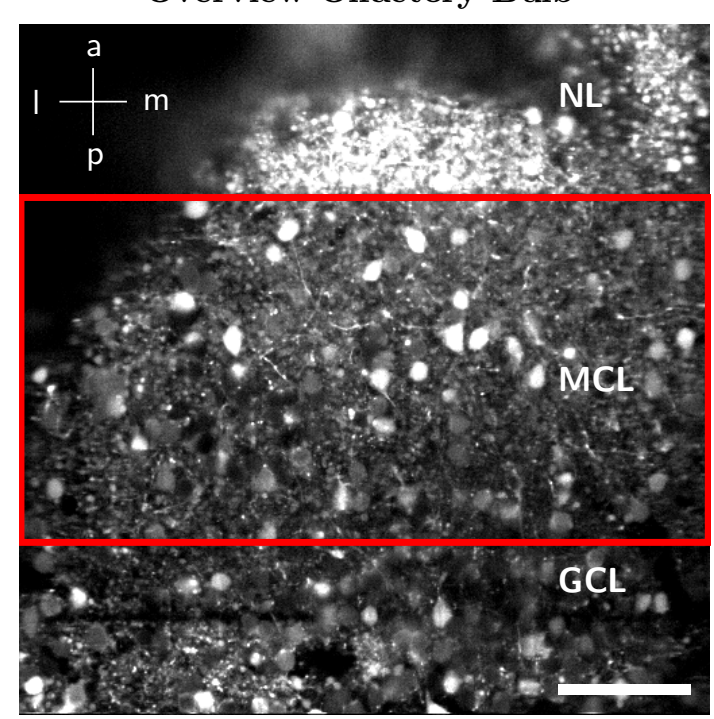

d

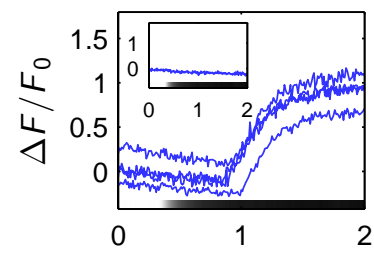

$\mathbf{e}$

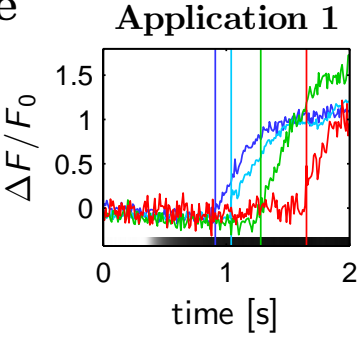

b

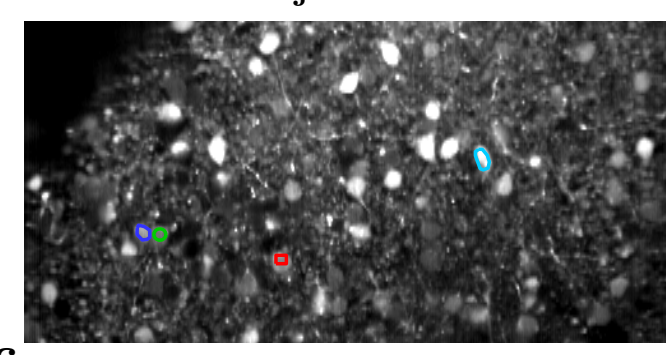

c

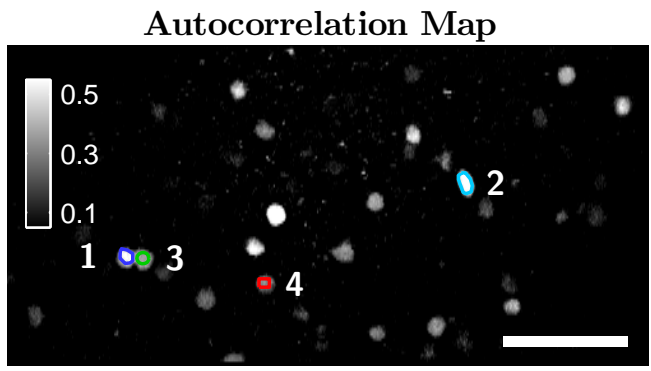

Cell 3

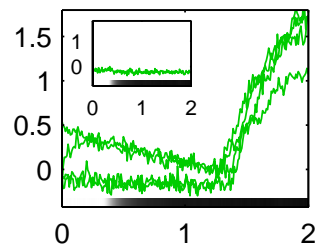

Application 3

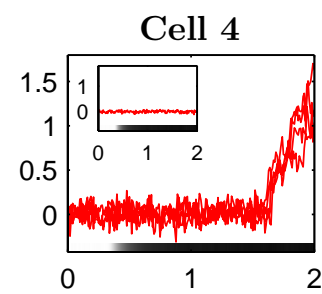

Application 4
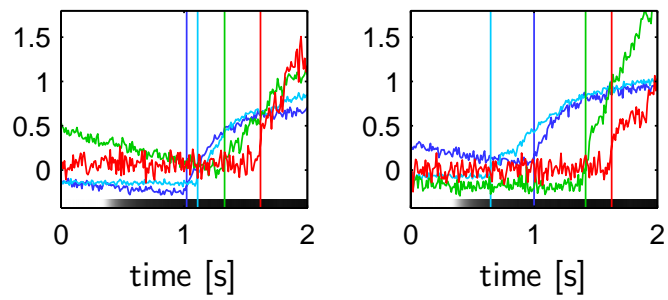

Figure 3.17: Investigation of odor-evoked latency patterns in the olfactory bulb. (a) Overview of one hemisphere of the olfactory bulb, stained with the $\mathrm{Ca}^{2+}$ sensitive fluorescent dye Fluo-4 (compare to organization of the olfactory bulb in Fig. 1.2). The region used for imaging (red outline) is placed in the mitral cell layer. Scale bar, $50 \mu \mathrm{m}$. (b) Mean projection over time of the image series of an actual experiment. (c) Selection of neurons that respond to a stimulus is facilitated by calculating an 'autocorrelation map'. Four somata that respond to the stimulus (arginine, $25 \mu \mathrm{M}$ ) are indicated. Scale bar, $50 \mu \mathrm{m}$. (d) Responses of the four neurons indicated in (c) to repeated applications of the amino acid arginine $(25 \mu \mathrm{M})$. The timing of the responses is highly reproducible. Insets: time traces during application of ringer solution (negative control). Horizontal bars at the bottom of each graph indicate stimulus application (gray values correspond to averaged values from Fig. 3.15b). (e) The same traces as in (d), but sorted by application. The vertical lines indicate the response onsets. The order of the onsets is very reproducible. Only the fourth application shows a deviation from the pattern.

Abbreviations a: anterior, p: posterior, l: lateral, m: medial, NL: nerve layer, MCL: mitral/tufted cell layer, GCL: granule cell layer. 

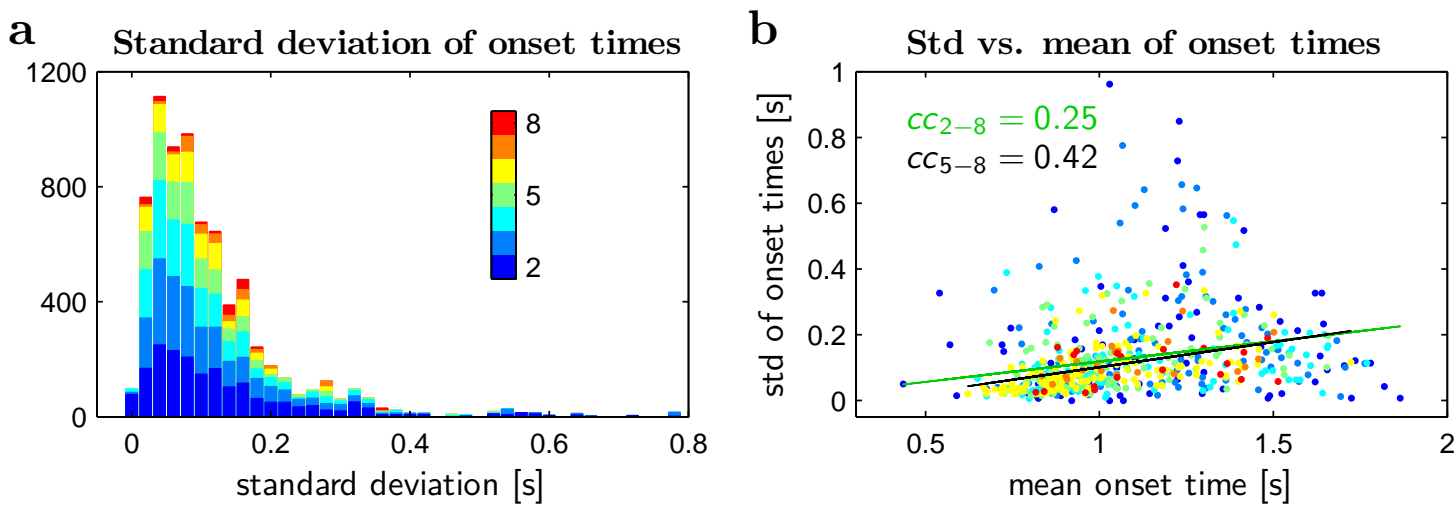

Figure 3.18: Variability of response onset times. (a) For each neuron and each stimulus, the standard deviation of the response onset time was calculated. The distribution has a peak around $40 \mathrm{~ms}$ and a median of about $84 \mathrm{~ms}$. As a comparison, the standard deviation of the odor application system is around $25 \mathrm{~ms}$. The onset times across neurons and stimuli have a standard deviation of almost $300 \mathrm{~ms}$. Colors indicate the number of applications that were included in the calculation of the standard deviation values. (b) To test whether the variability of the onset times depends on the absolute latency, the standard deviation was plotted as a function of mean onset time for each cell and each stimulus. There is a positive correlation between these parameters, as quantified by the correlation coefficient $\left(c c_{2-8}\right.$ : correlation coefficient based on all values, $c c_{5-8}:$ correlation coefficient of values that are based on at least five onset times.) The lines indicate the respective linear regressions. Despite this general trend, some neurons with a long latency show a remarkably small standard deviation.

Abbreviation std: standard deviation.

of the onset time is related to the absolute latency, the standard deviation of the onset times for each cell and each stimulus was plotted against the mean onset time (Fig. 3.18b). The distribution indicated a weak positive correlation, which was confirmed by calculating the correlation coefficient $\left(c c_{2-8}=0.25\right)$. Using only the statistically reliably values that are based on at least 5 onset times, gives an increased correlation coefficient of $c c_{5-8}=0.42$. This observation might reflect the co-variance between latency and standard deviation reported for receptor neurons (Gomez and Atema, 1996). Despite this general trend, some cells with a long latency show a remarkable reproducibility in their onset times (see also cell 4 in Fig. 3.17d), indicating that the $\mathrm{M} / \mathrm{T}$ cell onset pattern is not simply a copy of ORN activation 
a

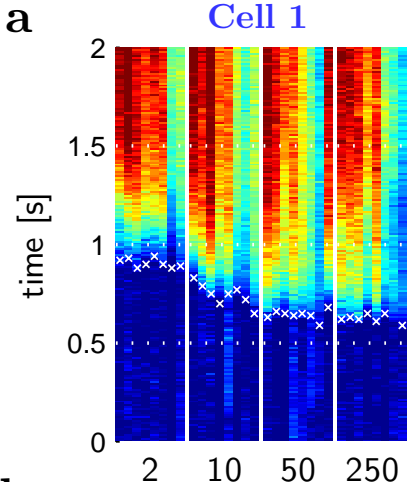

b

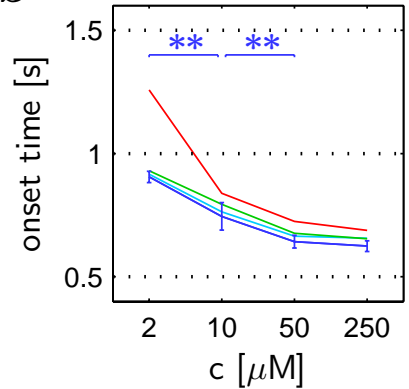

Cell 2
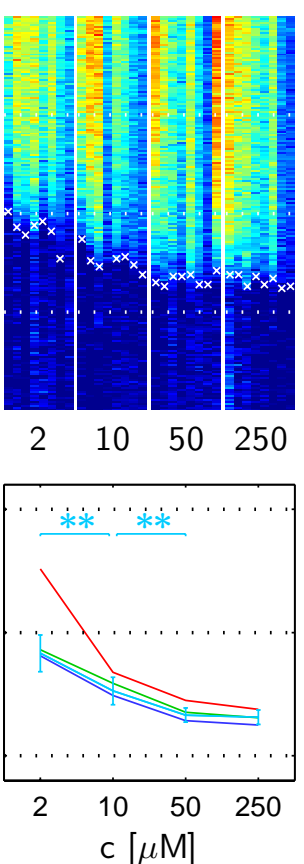

Cell 3
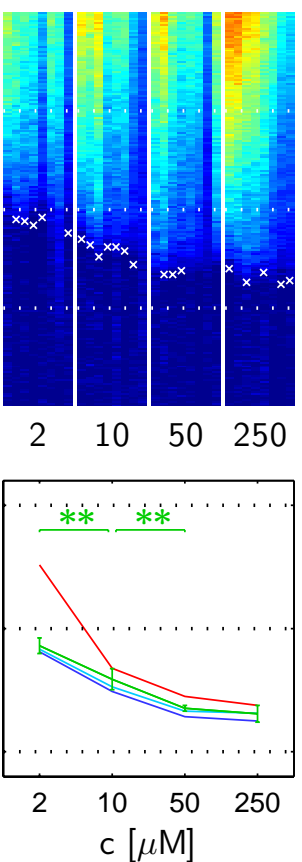

Cell 4
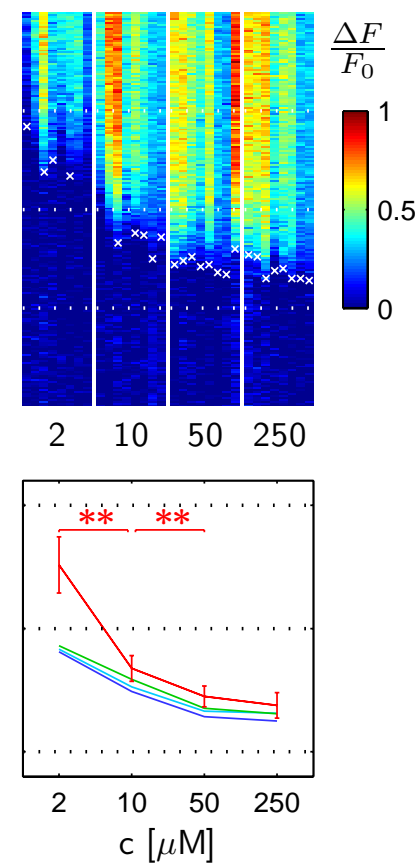

Figure 3.19: Concentration dependence of response onset times. (a) Shown are the responses of four neurons from the same slice as $\Delta F / F_{0}$ values (see colorbar). Each plot consists of columns depicting the response patterns of a given neuron to eight applications of the odor arginine at the indicated concentration (in $\mu \mathrm{M}$ ). The white ' $x$ '-symbols indicate the response onset. (b) Each graph depicts the mean values and standard deviations of the onset times of the respective neuron above it, and the mean values only for the other three cells. For all neurons, the response latency decreases with increasing concentration. This change is statistically significant $(* *: p<0.01)$. Additionally, it appears that the onset order across neurons is independent of concentration.

patterns.

To investigate whether the population onset patterns contain information about the odor identity, the reproducibility and concentration dependence of onset times for a fixed odor (arginine) were investigated. An odor specific feature of the $\mathrm{M} / \mathrm{T}$ cell response pattern has to be invariant with respect to odor concentration. Figure 3.19a shows the responses of four $\mathrm{M} / \mathrm{T}$ cells to four different concentrations of the same odor, each being applied eight times. The selected concentrations cover the dynamic range of effective stimuli (Czesnik et al., 2003; Manzini et al., 2007a). The $\Delta F / F_{0}$ time courses are displayed as color coded columns. The onset times (indicated by 
Corr. coef. w/o randomization

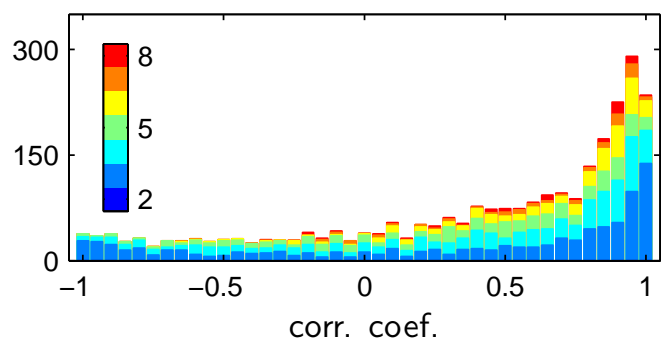

Corr. coef. with randomization

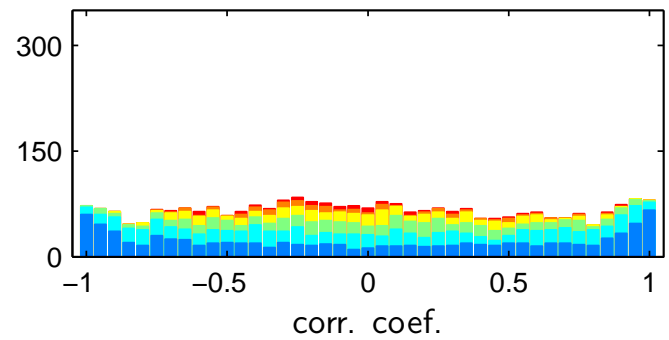

Figure 3.20: Inter-neuronal correlations between onset times across trials. (a) To determine whether the fluctuations in onset times are correlated between neurons, the correlation coefficients of onset times was calculated for all pairs of neurons within a slice over all applications of each stimulus. The distribution shows a peak around 0.95 , indicating that the fluctuations in onset times are strongly correlated. Colors indicate the number of applications that were included in the analysis. Coefficients based on less than three applications were excluded from the display. (b) As a comparison, the correlation coefficients were calculated after randomizing the trial order, in this way removing any causal relations between cells. The peak at large coefficients disappeared, the distribution appears nearly uniform (same color-code as (a)). This observation emphasizes the necessity to measure populations of neurons simultaneously.

the ' $X$ ') show that the response latency decreases with increasing concentration for all cells (Fig. 3.19b). The concentration dependent differences in onset times cover a range of typically about $200 \mathrm{~ms}$ and they are significant for all pairs of concentrations except $50 \mu \mathrm{M}$ vs. $250 \mu \mathrm{M}$ (Student's t-test, $p<0.01$, see Fig. 3.19b). The onset order (vertical order of lines in Fig. 3.19b) is in addition very similar across concentrations. The population onset pattern appears to be a concentration independent feature of the overall spatio-temporal response pattern, and therefore a promising candidate for a stimulus specific characteristic.

While the concentration dependence of the absolute response latency was demonstrated unambiguously by using the averages of the response onset times, the evaluation of the order of response onsets is less clear even for only four cells (Fig. 3.19). Larger numbers of neurons make it impossible to draw a conclusion about the reproducibility of the onset order or inter-neuronal delay times based on mean values and standard deviations of the response onset times. Another way of quantifying 
the odor-evoked population responses is therefore required.

\subsubsection{Measures for the similarity of population onset times}

Scientific research commonly uses averages of values that result from various trials of a given experiment (e.g. repeated applications of the same stimulus). This serves to emphasize the characteristic features that are contained in the behavior of the observed system and to suppress random variability. The activity patterns in the olfactory bulb are however assumed to rely on the temporal dependencies of the activity of the individual neurons. The absolute response times of individual cells is thus unlikely to carry the relevant information. Rather, the odor specific information is contained in the relative timing of neuronal activity within the network. Trialto-trial fluctuations of the absolute times of activity might result from variability in the experimental setup (e.g. fluctuations in the time of stimulus onset) or due to a variability of the temporal behavior of parts or the complete network. To determine whether the variance of neurons within a slice co-vary, the correlation coefficient between pairs of cells across applications of the same stimulus were calculated:

$$
c c_{(i, j)}=\frac{\sum_{a}\left(t_{i}^{\text {on }}(a)-\overline{t_{i}^{\text {on }}}\right) \cdot\left(t_{j}^{\text {on }}(a)-\overline{t_{j}^{\text {on }}}\right)}{\left\|t_{i}^{\text {on }}(r)-\overline{t_{i}^{\text {on }}}\right\| \cdot\left\|t_{j}^{\text {on }}(r)-\overline{t_{j}^{\text {on }}}\right\|} .
$$

The distribution of correlation coefficients, shown in Figure 3.21a, is skewed towards large values with a peak around 0.95. As a comparison, the correlation coefficients were calculated after randomizing the onset times of the neurons, thereby removing any causal covariance. The resulting distribution (Fig. 3.21b) is close to a uniform distribution, emphasizing the existence of a covariance in the onset times of the neurons. This observation is only possible due to the simultaneous recording of a large number of neurons, and emphasizes the need to investigate inter-neuronal dependencies in response patterns of single trials, rather than comparing inter-trial observations. The observed covariance in $\mathrm{M} / \mathrm{T}$ cell responses obscures the presumed invariant patterns of population dynamics when averaging absolute response times for individual neurons before investigating inter-neuronal dependencies. It is thus crucial for the analysis of neuronal network dynamics to investigate the dependencies of the neuronal activities on a trial-by-trial basis, and to use averages only for quantities that concern the population dynamics and do not depend on an absolute 
a

Application 1

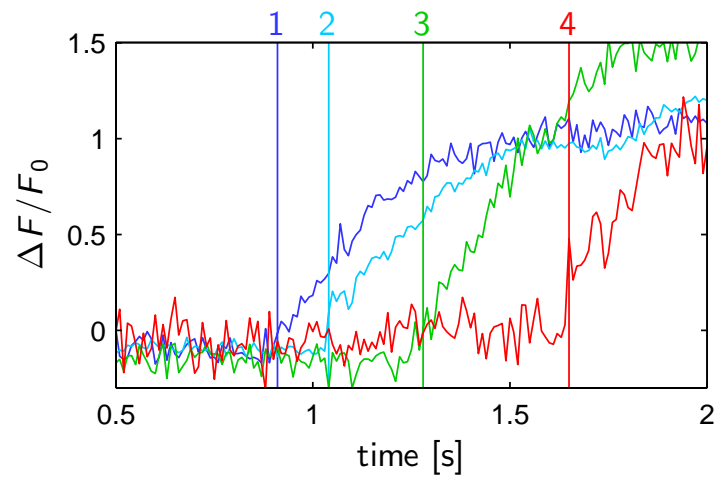

b

Correlation Coefficient

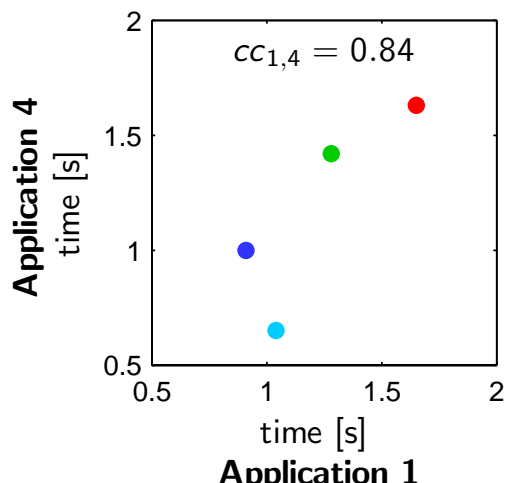

Application 4

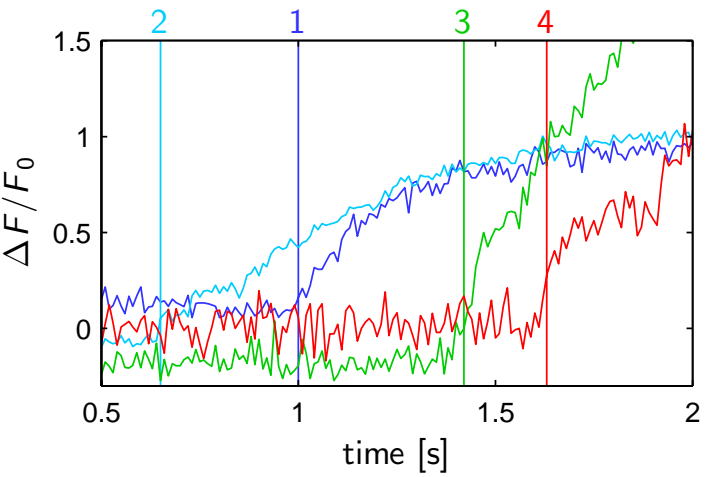

C

Inversion Index

Onset order Appl. 1

$\left(\begin{array}{llll}1 & 2 & 3 & 4\end{array}\right)$

Onset order Appl. 4

( $\left.\begin{array}{llll}2 & 1 & 3 & 4\end{array}\right)$

Number of inversions

$N_{1,4}^{\operatorname{lnv}}=1$

Max. number of inversions $N_{\max }^{\operatorname{lnv}}(4)=6$

Inversion index $\quad I_{1,4}=1-1 / 6=0.83$

Figure 3.21: Two measures for the similarity of latency patterns between odor applications within the same slice are introduced, the correlation coefficient and the inversion index. (a) Shown are the relative $\mathrm{Ca}^{2+}$ time courses of four neurons to two odor applications and the respective response onsets (vertical lines; same data as in Fig. 3.17d, Appl. 1 and 4). While the correlation coefficient is based on the time of the onsets, the inversion index uses the relative order in which the cells start responding (cell order indicated by colored numbers on top of graphs). (b) Plotted are the onset times of the four cells during application 2 against the onset times during application 1. The correlation coefficient measures the 'linearity' of the depicted pairs of onset times. Since the values form approximately a straight line (with the exception of cell 2, cyan point), the resulting correlation coefficient $c c_{1,4}$ is close to 1 . (c) The inversion index is based on the number of inversions between the two 'order-of-onset' vectors. In this example, only the neurons 1 and 2 exchanged their position, i.e. there is only one inversion. This value is then normalized to the maximum number of inversions for the respective number of cells (in this case a maximum of 6 inversions) and then subtracted from one. 
time reference. To this end, a measure for the similarity between the population onset patterns of two neuronal response patterns has to be defined. This measure can be used to compare the response onsets for pairs of applications using either the same or different stimuli. Averages of this measure can be calculated for each pair of stimulus conditions (stimulus A vs. stimulus A; stim. A vs. stim. B; stim. B vs. stim. B; etc.).

Two measures are introduced to compare the population onset patterns between pairs of odor application experiments within the same preparation. These measures are based on the response onset times $t_{i}^{\text {on }}(r)$ and $t_{j}^{\text {on }}(r)$ of the $i^{\text {th }}$ and $j^{\text {th }}$ application, respectively, for neurons $r \in R, R$ being the set of all $\mathrm{M} / \mathrm{T}$ cells within the field of view. Not all neurons might respond during each application and for some responsive neurons the onset time might not be accessible (see 3.3.2). For each pair of applications, the intersection $R_{i, j} \subseteq R$ of neurons with available onset times is used for the quantification of the response similarity. The number of elements in $R_{i, j}$ can be different for each application pair $(i, j)$. The traces of the applications 1 and 4 in Figure 3.17e will be used to illustrate the proposed measures (Fig.3.21a).

\section{Correlation coefficient}

The correlation coefficient $C_{i, j}$ can be used to measure the similarity of the response onset times $t_{i}^{\text {on }}(r)$ and $t_{j}^{\text {on }}(r)$ of a set of neurons from two odor applications $i$ and $j$ (Fig. 3.21b). $C_{i, j}$ is defined as (Beichelt and Montgomery, 2003)

$$
C_{i, j}=\frac{\sum_{r \in R_{i, j}}\left(t_{i}^{\text {on }}(r)-\overline{t_{i}^{\text {on }}}\right) \cdot\left(t_{j}^{\text {on }}(r)-\overline{t_{j}^{\text {on }}}\right)}{\left\|t_{i}^{\text {on }}(r)-\overline{t_{i}^{\text {on }}}\right\| \cdot\left\|t_{j}^{\text {on }}(r)-\overline{t_{j}^{\text {on }}}\right\|} .
$$

This value is a measure for the degree of linearity between the pairs of onset times of these neurons (Beichelt and Montgomery, 2003). While this value can be a useful indicator for onset similarity, it is very sensitive to extreme values. If, for example, a neuron consistently responds with latencies much longer than the other neurons, the correlation coefficient will assume artificially large values, regardless of the relation between the remaining onset times. 


\section{Inversion index}

Thus another measure is introduced that exclusively assesses the similarity of the onset order, while disregarding the durations by which the onset times are separated. To this end, the neurons of a slice are numbered in an arbitrary way. For each application, the numbers of the responding neurons are sorted in the order of the respective onset times (see colored numbers on top of the graphs in Fig. 3.21a). The similarity of two onset patterns is now defined by the degree to which the cells appear in the same order in these two permutations (Fig. 3.21c). This similarity can be quantified by counting the pairs of cells that appear in a different order in the two sequences, i.e. the number of inversions $N^{\text {Inv }}$ (Weisstein, 2002). Inversion based measures are commonly used to assess the similarity of genomic sequences (Watterson et al., 1982). In the example from Figure 3.21, the only inversion is found in the first two positions of the permutation vectors for the applications 1 and 4, yielding $N_{1,4}^{\mathrm{Inv}}=1$. The range of this value depends on the length of the sequence, i.e. on the number of elements in $R_{i, j}$. It is desirable to create an index that is independent of this number and that assumes large values for a high degree of similarity. Thus the inversion index $I_{i, j}$ for the application pair $(i, j)$ is introduced, defined as

$$
I_{i, j}=1-\frac{N_{i, j}^{\operatorname{Inv}}}{N_{\max }^{\operatorname{Inv}}\left(L_{i, j}\right)},
$$

with $N_{\max }^{\mathrm{Inv}}\left(L_{i, j}\right)$ being the maximum number of inversions for a sequence with length $L_{i, j}$, which is given by $N_{\max }^{\mathrm{Inv}}\left(L_{i, j}\right)=L_{i, j} \cdot\left(L_{i, j}-1\right) / 2$. This normalization yields a range that is independent of the sequence length $(I \in[0,1]$ for all $L)$. The example in Figure 3.21 yields $L_{1,4}=4, N_{\max }^{\mathrm{Inv}}(4)=6$ and thus $I_{1,4}=1-1 / 6=0.83$. It can be shown that $N_{\text {Inv }}$ is a metric on the space of all permutations of the numbers $\{1, \ldots, L\}$, justifying the use of the inversion index as a 'measure of similarity' (inverse distance measure) in a mathematical sense.

Both the inversion index and the correlation coefficients are measures of neuronal interdependencies of population response onset. They are thus independent of an absolute time frame. It should however be acknowledged, that an inversion index based on a long sequence of neurons contains more information than an index based on a short sequence. For example, a pair of identical sequences of length ten is 
less likely to occur by chance than a pair of identical sequences of length two. For the display of inversion indices, color-coded stacked histograms can be used (see Fig. 3.22). Each histogram visualizes the distribution of inversion indices, which are pooled into bins of equal width (bin width is 0.1 for inversion index histograms throughout this thesis). The counts within each bin are segregated according to the underlying distribution of sequence lengths. This distribution is visualized by stacked color patches, with the height of each segment representing the number of counts and the color representing the sequence length (see colorbar in Fig. 3.22 and 3.23). The same visualization is used for the display of the correlation coefficients (see for example Fig. 3.22e). When calculating average values, the sequence length can be accounted for by using a weighted mean instead of the common (unweighted) arithmetic mean. In this way, values based on longer sequences will have a stronger 'impact' on the resulting average value than those based on short sequences. It is desirable that the weighted mean $\tilde{I}$ has the same range as $I$, the weight factor is therefore chosen to be the maximum number of inversions $N_{\max }^{\mathrm{Inv}}$. This results in the weighted mean inversion index

$$
\tilde{I}=\frac{\sum_{i, j} I_{i, j} \cdot N_{\max }^{\operatorname{Inv}}\left(L_{i, j}\right)}{\sum_{i, j} N_{\max }^{\operatorname{Inv}}\left(L_{i, j}\right)}=1-\frac{\sum_{i, j} N_{i, j}^{\mathrm{Inv}}}{\sum_{i, j} N_{\max }^{\operatorname{Inv}}\left(L_{i, j}\right)},
$$

with $N_{i, j}^{\mathrm{Inv}}$ and $N_{\max }^{\mathrm{Inv}}\left(L_{i, j}\right)$ being the number of inversions and the maximum possible number of inversions for the application pair $(i, j)$ with sequence length $L_{i, j}$, respectively. This definition of $\tilde{I}$ is consistent with the definition of $I$ in that (1) $\tilde{I} \in[0,1]$ and (2) if all sequences have the same length $L_{i, j} \equiv L, \tilde{I}$ is becoming the regular average of the inversion index $\tilde{I}=\bar{I}=\frac{1}{K} \sum_{i, j} I_{i, j}$. Similarly, a weighted standard deviation $\widetilde{\sigma}_{I}$ is defined,

$$
\tilde{\sigma}_{I}=\sqrt{\frac{\sum_{i, j}\left(I_{i, j}-\tilde{I}\right)^{2} \cdot N_{\max }^{\operatorname{Inv}}\left(L_{i, j}\right)}{\sum_{i, j} N_{\max }^{\operatorname{Inv}}\left(L_{i, j}\right)}} .
$$

For the correlation coefficient, the sequence length $L_{i, j}$ is used as a weight. This yields the following definitions for the weighted mean $\widetilde{C}$ and standard deviation $\widetilde{\sigma}_{C}$ of the correlation coefficient, respectively:

$$
\widetilde{C}=\frac{\sum_{i, j} C_{i, j} \cdot L_{i, j}}{\sum_{i, j} L_{i, j}} \quad \text { and } \quad \tilde{\sigma}_{C}=\sqrt{\frac{\sum_{i, j}\left(C_{i, j}-\widetilde{C}\right)^{2} \cdot L_{i, j}}{\sum_{i, j} L_{i, j}}} .
$$


In the following, both measures will be used to measure the similarity between onset patterns, with a focus on the inversion index.

\subsubsection{Concentration and stimulus dependence of the inversion index $I$}

\section{Concentration dependence of $\mathbf{I}$}

Using the inversion index and the correlation coefficient, the similarity of the patterns of $\mathrm{M} / \mathrm{T}$ cell onset times for applications of different concentrations $(5 \mu \mathrm{M}$, $25 \mu \mathrm{M}, 125 \mu \mathrm{M})$ of the amino acid arginine was quantified. Figures $3.22 \mathrm{a}$ and $\mathrm{b}$ show the distributions of inversion indices for pairs of concentrations for a single slice and pooled over slices $(n=8)$, respectively, arranged in a matrix-like display. The color code of the histograms indicates which fraction of the distributions are due to shorter (cold colors) or longer (warm colors) sequences. Both diagonal (i.e. pairs of identical concentration) as well as off-diagonal distributions are centered around large values $(0.7-0.9)$, with virtually no entries for values smaller than 0.5. To facilitate the comparison of the distributions between same vs. different concentrations, the data from Figure $3.22 \mathrm{~b}$ are combined accordingly into the two histograms in Figure 3.22c. To appreciate the significance of the pattern similarities both for conditions of identical as well as different concentration, distributions of inversion indices were calculated for randomly generated sequences of onset order (using the same number of 'experiments' and distributions of sequence length). These histograms (Fig. 3.22d) are centered around the value 0.5. Especially for long sequences (warm colors), the probability of randomly generating two highly similar patterns twice is virtually zero, as reflected by the histograms. This emphasizes the significance of the high degree of similarity between onset patterns from stimulations with identical or different concentrations of the same odorant.

\section{Odor specificity of latency patterns}

The reproducibility of the population onset patterns can have two reasons. It could either imply that the $\mathrm{M} / \mathrm{T}$ cell network contains 'early' and 'late' neurons, i.e. that the latency is cell specific and independent of odor identity (though concentration dependent as seen from Fig. 3.19). Alternatively, it could mean that the order is 
$\mathbf{a}$

Distributions for a single experiment

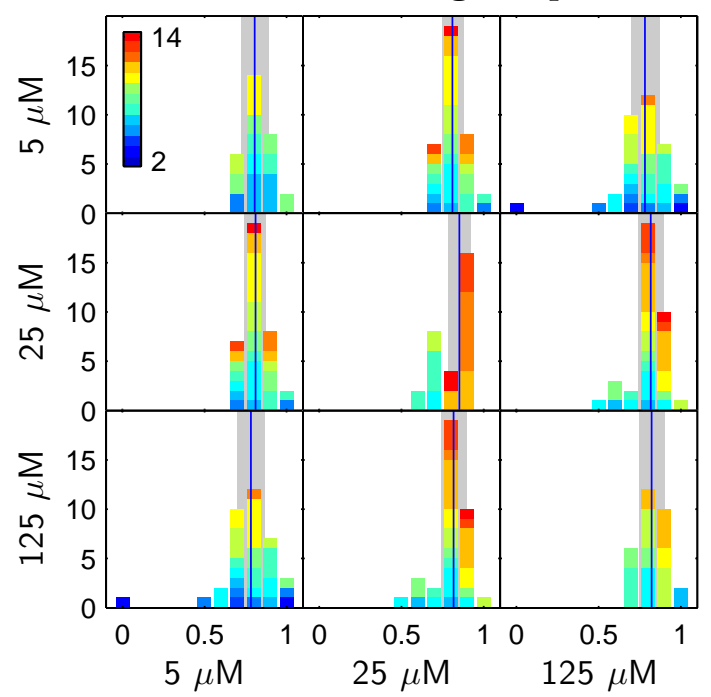

b

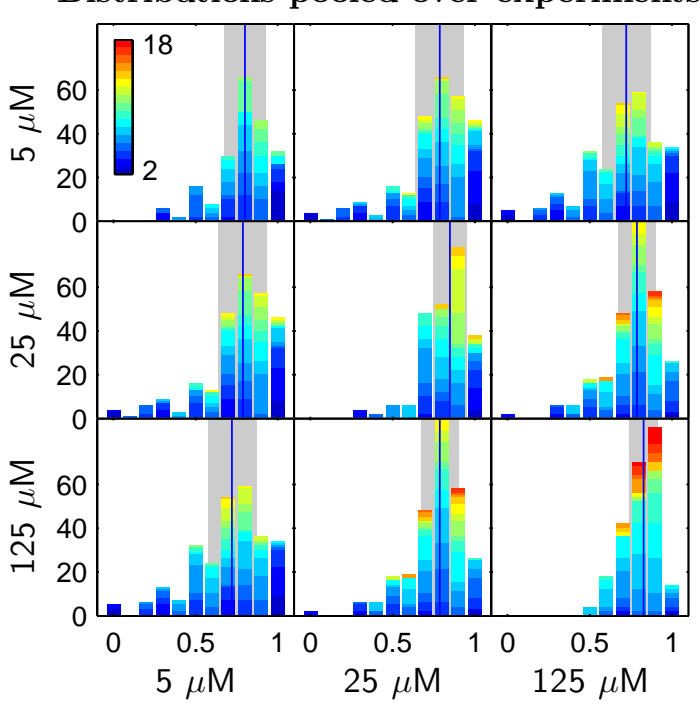

C
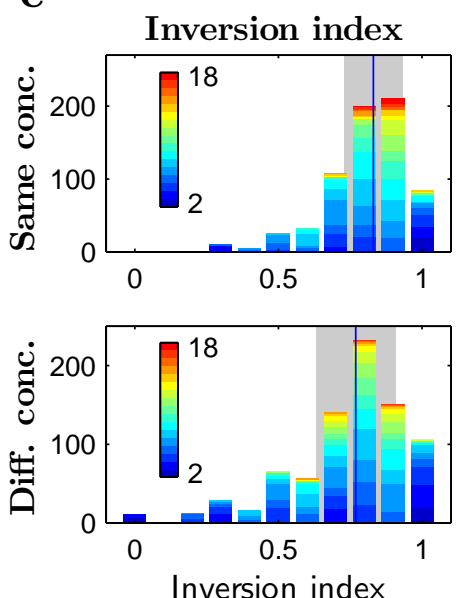

d
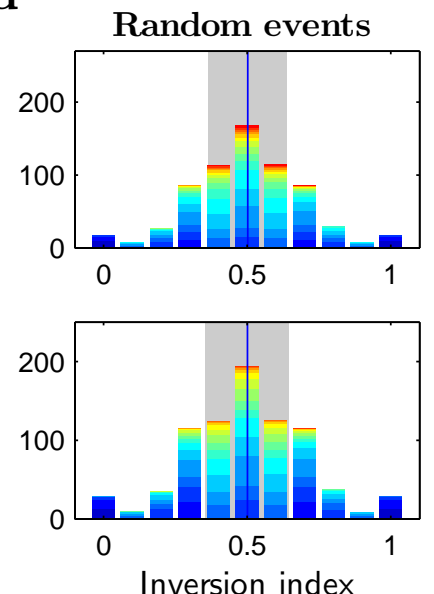

e
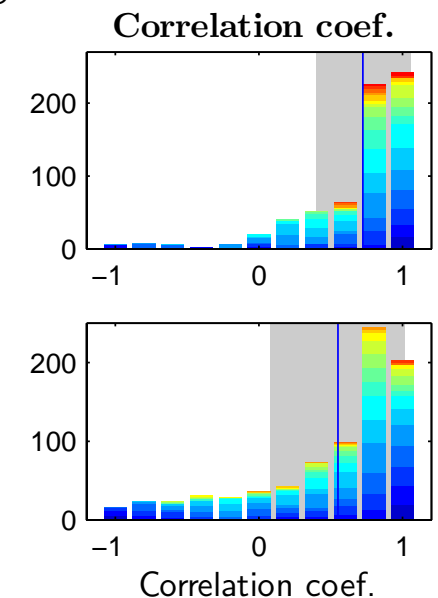

Figure 3.22: Distribution of inversion indices for applications of different concentrations of arginine. Arginine was applied repeatedly at three different concentrations and the inversion index was calculated for all pairs of applications. Blue vertical lines indicate the weighted mean, gray bars indicate mean \pm standard deviation (weighted according to sequence length, see text for details). (a) 'Matrix' of distributions of inversion indices for all pairs of concentrations for a single slice. Colors indicate the number of neurons underlying the calculated index (see colorbar). For pairs of both equal and different concentrations the distributions show peaks and mean values between 0.7 and 0.9, indicating a high reproducibility of the onset pattern. (b) Same display as in (a), but pooled results over eight slice preparations. The distributions are similar to (a), though with a larger standard deviation. (c) Inversion indices sorted for application pairs of equal (top) vs. different (bottom) concentrations. (d) For comparison, the distribution of inversion indices for randomized onset orders is displayed. These are centered around the value of 0.5. Note that especially for long sequences (warm colors), the probability for values close to one is almost zero. (e) Histograms of correlation coefficients of the same experiments used for the histograms in (c), sorted for application pairs of equal (top) vs. different (bottom) concentration. 
odor specific, and that a given cell $\mathrm{C}_{1}$ could have a shorter latency to odor $\mathrm{A}$ and a longer to odor $\mathrm{B}$, while the cell $\mathrm{C}_{2}$ shows a long latency in response to odor $\mathrm{A}$ and a shorter to odor B. If the former explanation was correct, the inversion indices comparing applications of different odors should be very similar to the inversion indices comparing applications of the same odor and small inversion indices should not occur. To test the hypothesis of odor independent onset patterns, experiments using applications of different amino acids (arginine, histidine, and phenylalanine) were conducted. Figure 3.23a and b show the distributions for all pairs of odors from a single slice and pooled over slices $(n=6)$, respectively. Comparing applications of identical odorants (histograms on the diagonal in Fig. 3.23a and b) yields similar distributions found the experiments concerning concentration dependence, with most values around $0.8-0.9$. Comparing on the other hand applications of different odors yields distributions centered around smaller values. The distributions appear gaussian shaped with an odor-pair specific mean value for experiments of a single slice. When pooling the results over several slices (Fig. 3.23b), the distributions for pairs of different odors appear broader and shifted toward smaller values, compared to the distributions for pairs of identical stimuli.

The degree of similarity between onset pattern presumably depends on the choice of odorants (see distributions in Fig. 3.23) and the preparation (as a random 'selection' of $\mathrm{M} / \mathrm{T}$ cells is in the field of view). It is nevertheless desirable to quantify and compare the similarities of ensemble patterns between stimulations with various concentrations of an odorant vs. stimulations with various odorants. The weighted mean and standard deviations indicated in Figures 3.22 and 3.23 are a first indicator that the onset patterns are more informative about odor identity than odor concentration. Due to the weights associated with each value (inversion index or correlation coefficient), a traditional significance test is however not possible. For this reason, the bootstrap sampling method was used (Boos, 2003; Efron, 1979). This method is based on resampling the data by drawing independent samples with replacement from a given data set. The statistics of this resampled set is computed (e.g. the weighted mean), and the procedure is repeated a larger number of times (1000 times for the results presented here). In this way a distribution of weighted 
$\mathbf{a}$

Distributions for a single experiment

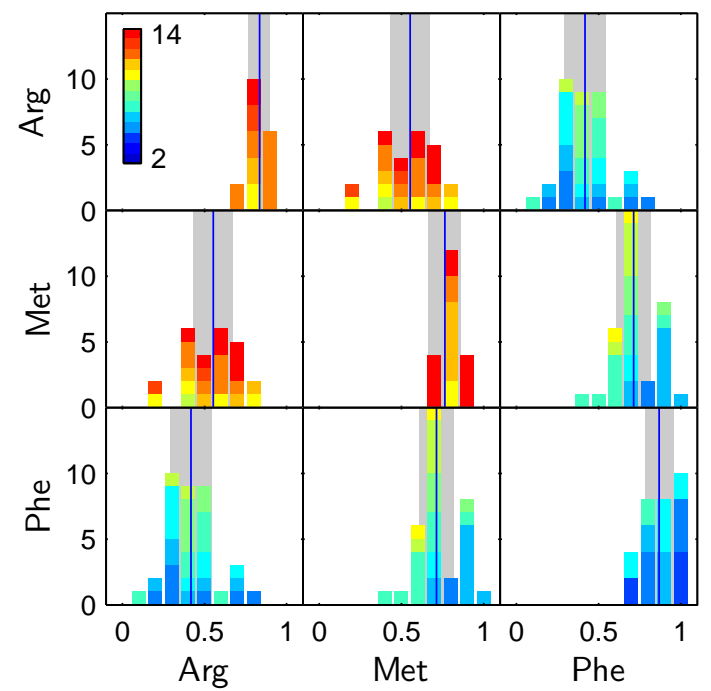

C
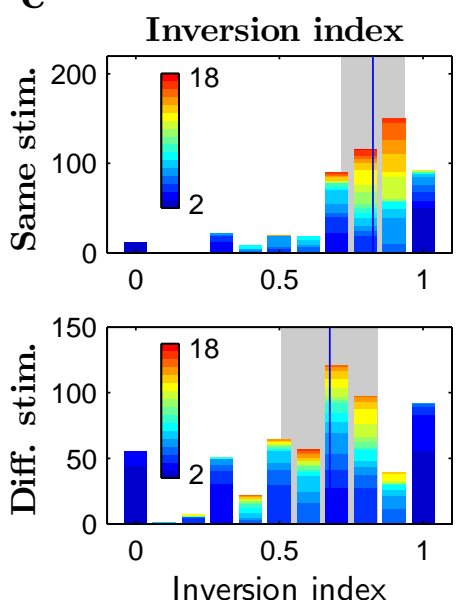

b

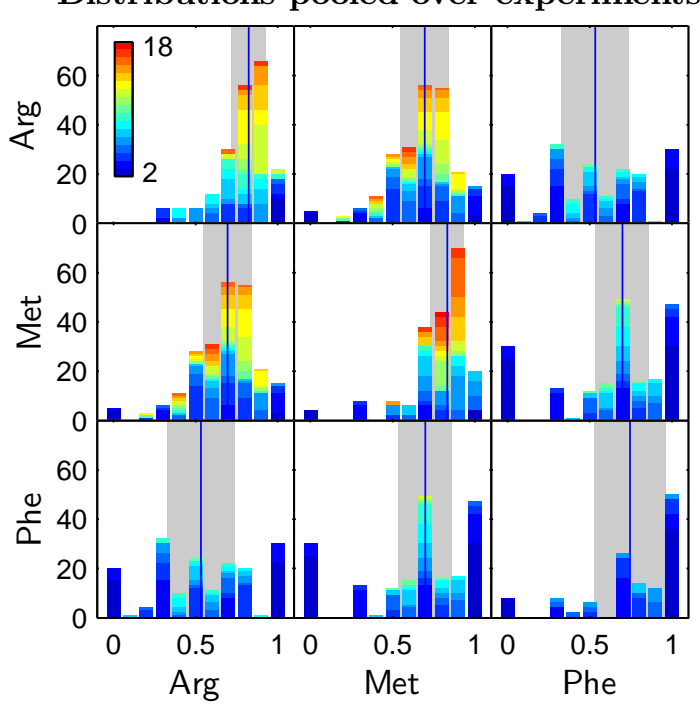

e
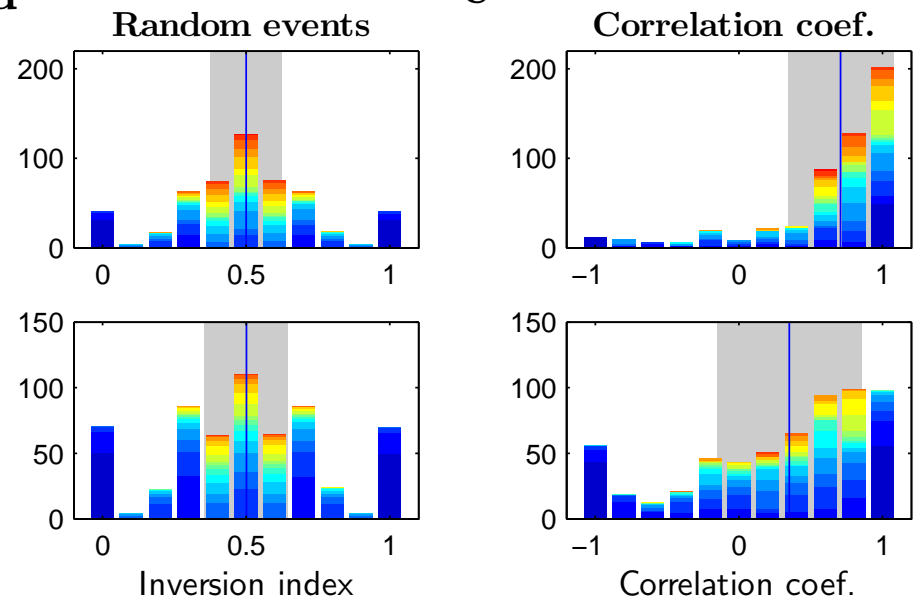

Figure 3.23: Distributions of inversion indices for applications of different odorants. Three different amino acids were applied repeatedly and the inversion index was calculated for all pairs of applications. (a) 'Matrix' of distributions of inversion indices for all pairings of odorants for a single slice. Different colors represent different numbers of neurons underlying the calculated index (see colorbar). The histograms along the diagonal are similar to the histograms shown in Fig. 3.22a. In contrast, the off-diagonal histograms show a shift to lower values. This shift appears to be odor pair specific, for some being larger (Arg / Phe), for others smaller (Met / Phe). (b) Same display as in (a), but pooled results over five slice preparations. (c) Inversion indices sorted for pairs of same (top) vs. different (bottom) odor. (d) For comparison, the distribution of inversion indices for randomized onset orders is displayed. (e) Histograms of correlation coefficients of the same experiments used for the histograms in (c), sorted for pairs of equal (top) vs. different (bottom) odors.

Abbreviations Arg: arginine, Met: methionine, Phe: phenylalanine 
means can be generated and used for statistical significance tests. Figure 3.24a and b show the results of the bootstrap analysis, performed for the inversion indices pooled over experiments using various concentrations and various stimuli, respectively. The distributions show, that the inversion index is largest for pairs of applications using the same stimulus, i.e. identical both in concentration and identity (centered around 0.83). Comparing applications using the same odor at different concentrations shows a significant $(p<0.01)$ but small reduction in inversion index (centered around 0.77). Comparing applications of identical concentration but different odor shows a significant $(p<0.01)$ and strong reduction in inversion index (centered around 0.67 ). The onset order of $\mathrm{M} / \mathrm{T}$ cell ensembles is thus highly reproducible for a given odor, and significantly more informative about the odor identity than about odor concentration.

Finally, the variability of inversion indices and correlation coefficients between different slice preparations was compared. To this end, the weighted mean of either measure was calculated for each slice, and the mean and standard error of these measures was calculated across slices $(n=8$ for various concentrations and $n=6$ for various stimuli). The results are shown in Figure $3.24 \mathrm{~b}$ and c. The calculated mean values are consistent with the bootstrap analysis, and the inter-slice variability is small. This demonstrates that the results are consistent across slices and thus represent a characteristic of the Xenopus laevis tadpole olfactory bulb.

\subsubsection{Preliminary results}

\section{Population onset patterns for complex odorants}

The presented experiments concerning reproducibility and specificity of odor-evoked population onset patterns were conducted using single amino acids as odorants. While these are known to be behaviorally relevant stimuli for tadpoles and other amphibians (Ferrer and Zimmer, 2007; Kiseleva, 1983, 1995), most natural odors are complex mixtures of single odorant molecules. Due to a broader activation of both receptor neurons and $\mathrm{M} / \mathrm{T}$ cells, interaction within the $\mathrm{OB}$ (via lateral connections by periglomerular and granule cells) might play a bigger role for shaping the spatiotemporal response patterns. It is thus not clear whether features of the response 
a

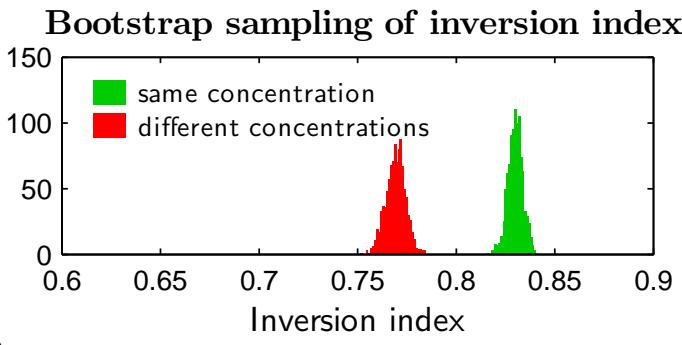

b

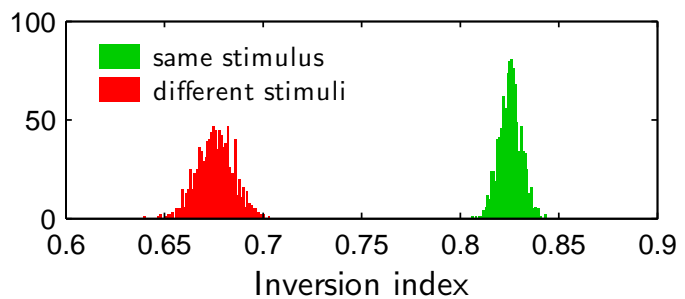

C

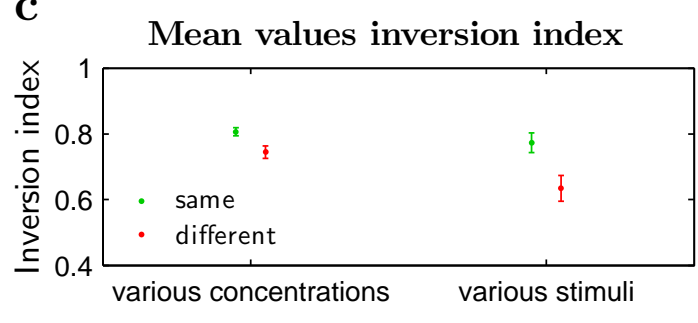

d

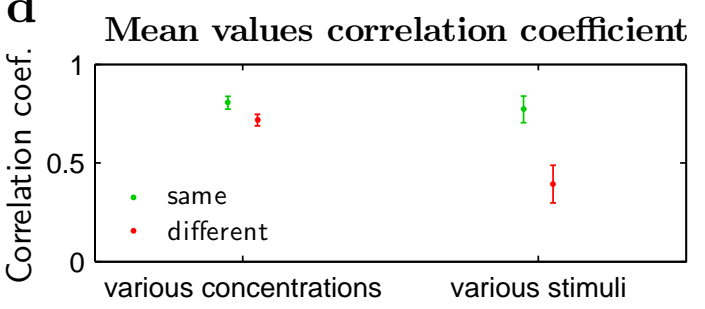

Figure 3.24: Statistical analysis of the results for the inversion index and the correlation coefficient. (a) Bootstrap sampling was performed to test for significance of the differences in inversion indices using various concentrations (see text for details). Based on this analysis, the inversion indices calculated for pairs of applications using the same concentrations (green) are slightly, but significantly larger than inversion indices calculated for pairs of applications using different concentrations (red). (b) Results of the bootstrap analysis for experiments using applications of various odorants. The inversion indices for pairs of applications using the same odorant (green) is significantly larger than for pairs of applications using a different odorant (red). This difference is much more pronounced than for experiments with variable odor concentration (a). (c) Mean values and standard error of inversion indices across slices. For each slice, the weighted mean of the inversion index was calculated for both conditions of identical and different concentrations / stimuli, and these values were averaged and the standard error was calculated. The results show, that the intertrial-variability is very small, and that the presented results represent general properties of the Xenopus olfactory bulb. (d) Mean values and standard deviations of the correlation coefficient across slices, for both conditions of identical and different concentrations / stimuli. The resulting values confirm the presented findings, additionally showing that the interslice-varibality is small.

patterns upon stimulation with mono-molecular odorants can be generalized to more complex odors. To investigate whether the reproducibility and specificity of population onset patterns can be found for stimulation with complex mixtures, a first experiment was conducted using a mixture of 15 amino acids and a food extract as 
a
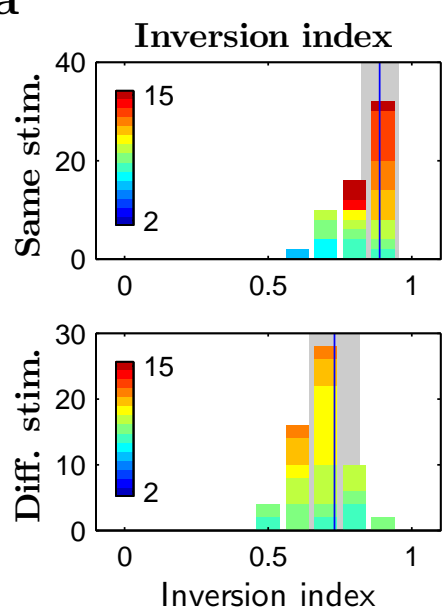

b
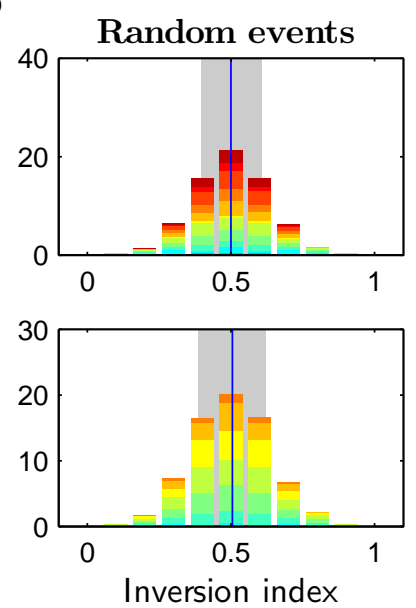

C
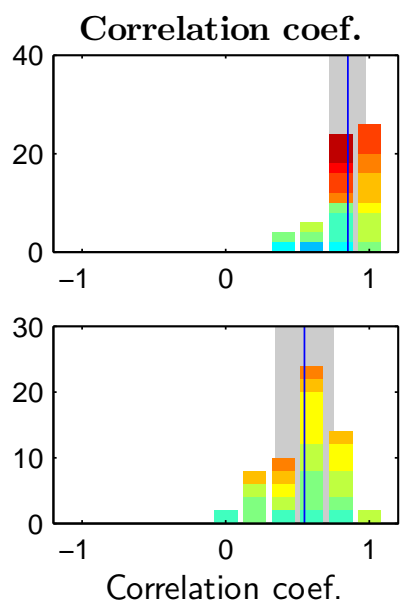

Figure 3.25: Distributions of inversion indices for applications of two complex mixtures. A mixture from 15 amino acids and a food extract were repeatedly applied and the inversion index was calculated for all pairs of applications. (a) Histograms of correlation coefficients sorted for pairs of equal (top) vs. different (bottom) odors. The bottom histogram shows a shift towards smaller values. (b) Inversion indices sorted for pairs of same (top) vs. different (bottom) odor. The prominent peak at 0.9 seen in the top histogram does not exist in the bottom graph. The values are shifted towards smaller values. (c) For comparison, the distribution of inversion indices for randomized onset orders is displayed.

stimuli. The results are presented in Figure 3.25 as distributions of inversion indices and correlation coefficients, similar to the display in Figure 3.23c-e. The results are very similar to the experiments using single amino acids, with a high similarity of onset patterns when comparing applications of the same stimulus (top row of histograms in Fig. 3.25), and with a significantly lower similarity when comparing applications of different stimuli (bottom row of histograms). This result shows, that the responses to complex mixtures show the same reproducibility and specificity of population onset patterns that was observed for simple odorants. This observation holds for both the inversion index and the correlation coefficient. This preliminary result (obtained in a single slice) has to be substantiated by further experiments. Additional experiments should address a potential correlation between the similarity of odors (chemical similarity for single molecules or with respect to similarity of 
$\mathbf{a}$

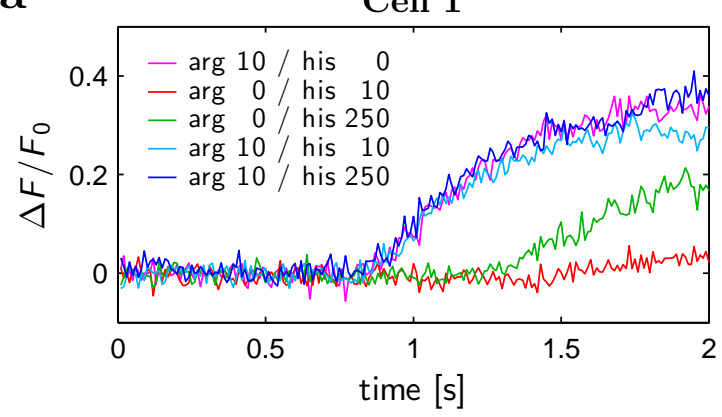

b

Cell 2

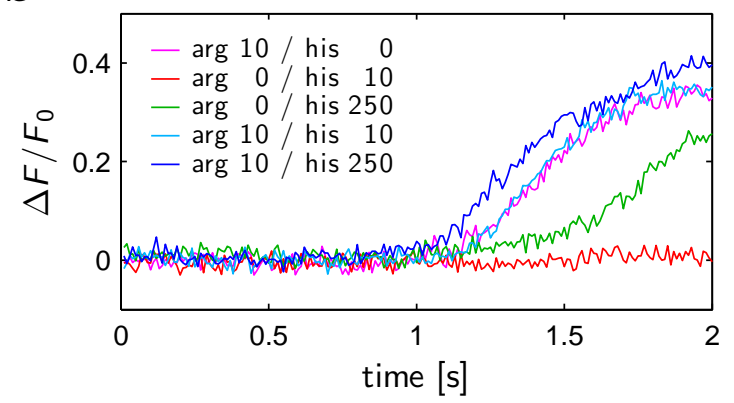

Figure 3.26: Preliminary results concerning response onset times in binary mixtures. Shown are representative examples of two neurons, responsive to both arginine (arg) and histidine (his). The amino acids were applied individually and in mixture, using a fixed concentration for arginine and two concentrations for histidine (see legends). Traces are averages over six applications of each stimulus. (a) While the neuron does not respond to low concentration of histidine $(10 \mu \mathrm{M})$, it responds with a long latency to a high concentration of histidine. The response to the mixtures appears always dominated by arginine, with no apparent difference in onset time. The onset time could in this case be predicted as the shortest onset time measured for the components. (b) The low histidine concentration is not an effective stimulus, and does not affect the response to an equally low concentration of arginine. The neuron responds (weakly) to higher concentration of histidine, and the mixture of the two effective stimuli show a reduced response latency compared to its components. The effect could be described as 'cumulative', as the effect of the two stimuli appears to add up.

mixtures for complex odors) and the similarity of population onset patterns.

\section{Response onset times for binary mixtures}

Most natural odors are complex mixtures of different types of molecules. The task of the olfactory system is to recognize and distinguish these complex odors, taking into account the contained components, as well as the ratios at which these are contained in the mixture. The responses to different components of an odor can be expected to interact in the OB. An understanding of these interactions and how the response to a mixture related to the responses to its components is crucial for our understanding of the coding principles of the OB. Much attention has consequently been payed to the investigation of the response patterns upon stimulation 
with simple odors and their mixture, in particularly using two mono-molecular odors and their binary mixture (see for example (Giraudet et al., 2002; Kang and Caprio, 1997; Khan et al., 2008; McNamara et al., 2007; Tabor et al., 2004)). For the investigation of population onset patterns, it is of interest to study the relation of the onset time of single neurons upon stimulation with either single odors or their binary mixture. A first step in this direction has been made with the amino acids arginine and histidine and their binary mixture, using two different concentrations of histidine. Figure 3.26 shows two representative examples of neurons responsive to either amino acid as well as to their mixture (response patterns averages over six applications of each stimulus). As expected, adding a subthreshold stimulus to an effective stimulus does not affect the response of the system $(10 \mu \mathrm{M}$ histidine in Fig. 3.25a,b). On the other hand, when mixing two effective stimuli (e.g., histidine $250 \mu \mathrm{M}$ and arginine $10 \mu \mathrm{M}$ ), the response to the mixture was found to be related to the responses to the components in one out of two ways: The latency when stimulating with the mixture was either similar to the shortest of the component latencies (Fig. 3.26a), or it was shorter than the shortest component latency ('cumulative effect', Fig. 3.26b). While these preliminary results are not exhaustive or conclusive, they indicate how latency patterns in complex odorants might be generated based on latency patterns of the components. Given the concentration dependence of the response latency, the system would be able to generate specific population onset patterns for mixtures that differ only in the mixing ratios of their components. However, more complex interactions of components of a mixture are very likely, given the lateral connectivity and the occurrence of inhibitory $\mathrm{M} / \mathrm{T}$ cell responses in the $\mathrm{OB}$. The question concerning coding of (binary) mixtures, and the relation between onset patterns for the mixture and its components, requires much attention and the investigation of a wide variety of odor pairs and their mixtures.

\section{Response latencies at the ORN level}

The existence of odor and concentration dependent response latencies raises the question about possible mechanisms that generate these specific latencies. An immediate question is, whether the latencies are already present at the level of ORNs 
$\mathbf{a}$

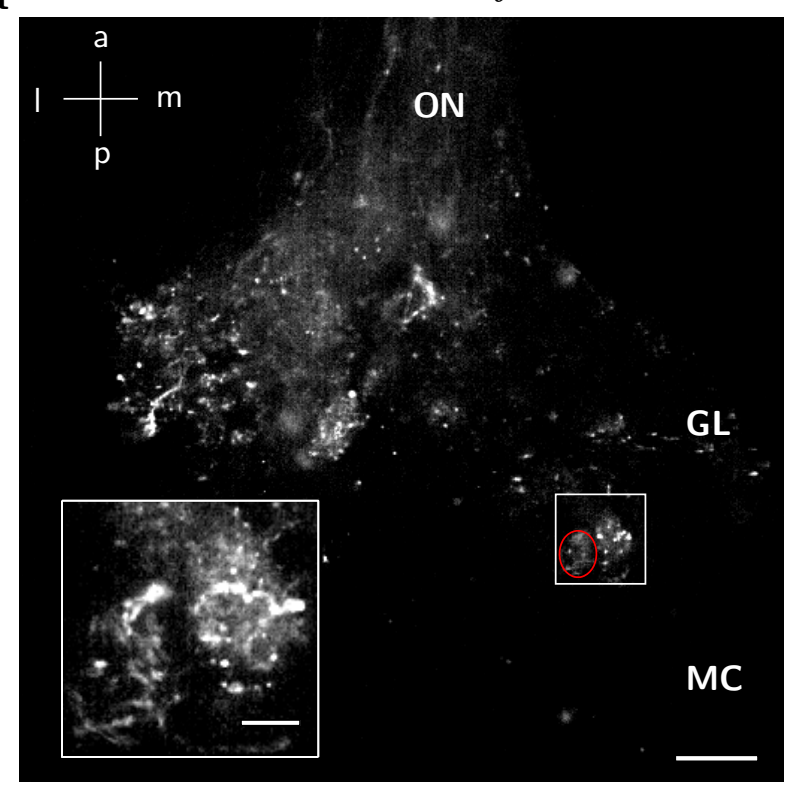

b Glomerular response

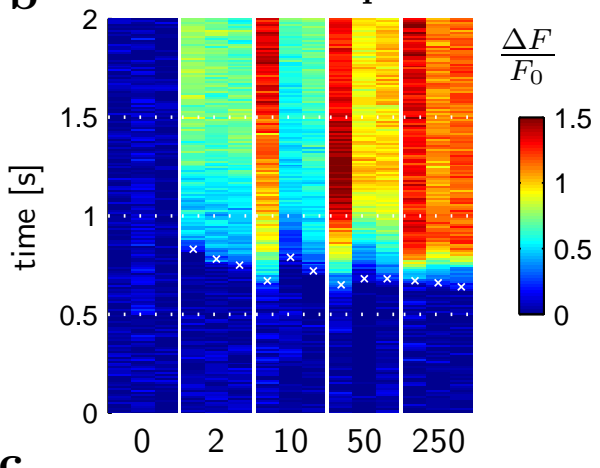

$\mathbf{C}$

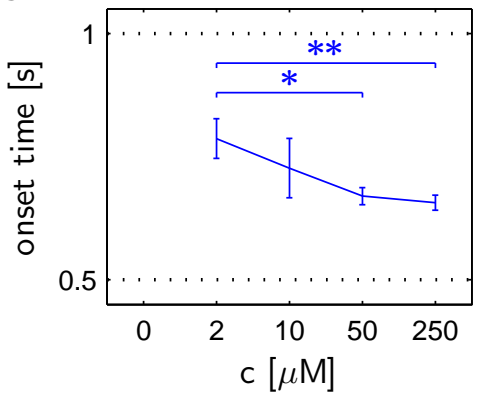

Figure 3.27: Concentration dependence of glomerular response onset times. (a) Overview of the OB. The ORNs have been filled with $\mathrm{Ca}^{2+}$ indicator dye, allowing to measure exclusively from glomerular terminals of ORN axons. The red ellipse outlines the glomerulus (shown magnified in the inset) that was responsive to the stimulus (mixture of 15 amino acids). Scale bar, $50 \mu \mathrm{m}$; scale in inset, $10 \mu \mathrm{m}$. (b) Responses to different concentrations of the odorant, including control measurement (three applications per concentration). The ' $x$ '-symbols indicate the response begin. (c) Averages and mean values of response onset times. The decrease in onset times with increasing concentration is statistically significant $(*: p<0.05, * *: p<0.01)$. The difference appears to be less pronounced than for M/T cells (Fig. 3.19).

or whether they are entirely generated by the circuitry of the OB. A novel preparation for the larval Xenopus laevis (established recently by Eugen Kludt, Department of Neurophysiology and Cellular Biophysics, University Göttingen) allows the investigation of ORN activity at the level of olfactory glomeruli. Using electophoresis, the ORNs are filled with $\mathrm{Ca}^{2+}$ sensitive fluorescent dye, which diffuses through the axon into the glomerular terminals. Using this preparation, it is possible to measure exclusively presynaptic glomerular signals. The size of the glomerular, their 
spatial distribution in the OB, and the optical sectioning of the LIM restricts the observation to only a few glomeruli at a time. Typically one or sometimes very few glomeruli within an optical slice will respond to a given stimulus. Therefore, the concentration dependence of the response latencies of individual glomeruli was investigated, instead of the population onset patterns across glomeruli. As it can be seen in Figure 3.27, the ORN response onset time is concentration dependent, with higher concentrations resulting in shorter latencies. The dependence appears in this example to be less pronounced than for $\mathrm{M} / \mathrm{T}$ cells (compare to Fig. 3.19), with larger differences in concentration necessary for a significant change in onset time. This preliminary data does not yet allow a quantification or a quantitative comparison with the findings in the OB. The result that the onset time of ORNs depends on the concentration of the stimulus is however in agreement with previous reports in other animals (Getchell and Shepherd, 1978; Gomez and Atema, 1996; Spors et al., 2006). While the existence of latencies at the level of ORN might be important for the dynamics of the $\mathrm{M} / \mathrm{T}$ cell responses, their existence does not imply that the latency patterns in the $\mathrm{M} / \mathrm{T}$ cells are simply a 'reflection' (i.e. a direct effect) of these latencies.

\section{Detection of action potentials from $\mathrm{Ca}^{2+}$ signals}

Parameters other than the population onset patterns might be relevant for olfactory coding (Bathellier et al., 2008). It would therefore be interesting to investigate further parameters, such as the spike frequency, the number of spikes or the modulation of spike patterns over time, and to analyze their interneuronal relations and dependencies. Different aspects of the response pattern might be strongly correlated (Margrie and Schaefer, 2003) or might code for different aspects of the stimulus (Friedrich et al., 2004). It is therefore desirable to assess and analyze the overall population activity, i.e. the patterns of APs in the population of $\mathrm{M} / \mathrm{T}$ cells in the OB. It has been shown, that APs are reflected as discrete steps in the $\mathrm{Ca}^{2+}$ signal (Smetters et al., 1999), as recorded using $\mathrm{Ca}^{2+}$ dependent indicator dyes at a sufficient temporal resolution. $\mathrm{Ca}^{2+}$ imaging is thus a suitable tool for recording APs in neuronal populations. The task is to reliably extract the times of APs from the 
a

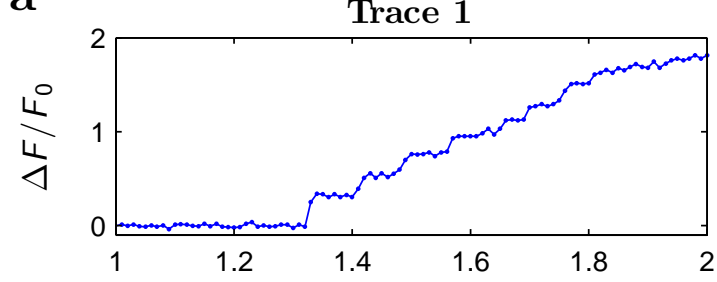

b Local correlation with step function

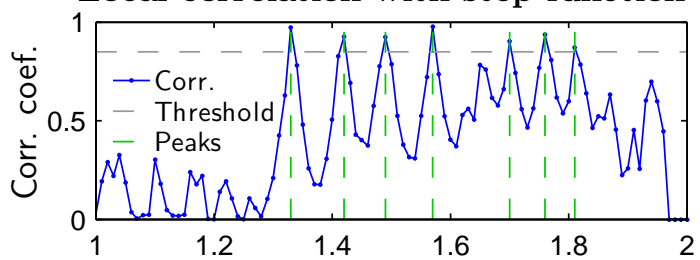

C

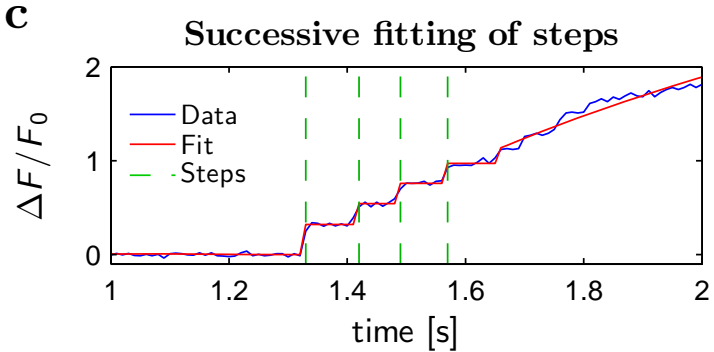

Trace 2

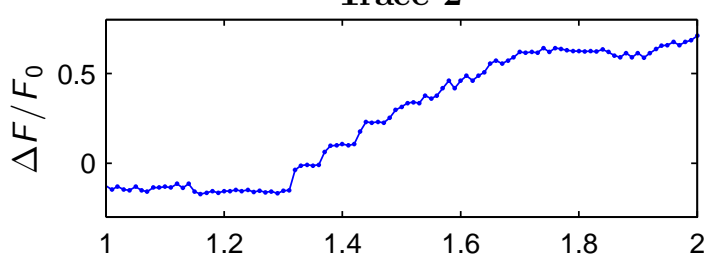

Local correlation with step function

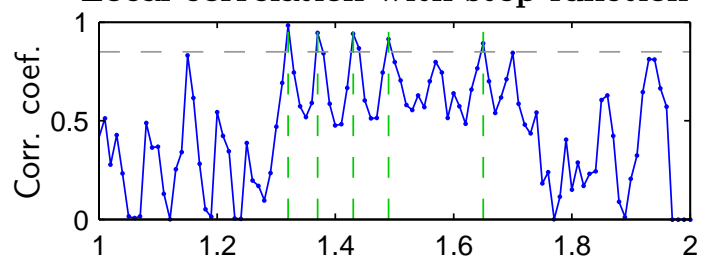

Successive fitting of steps

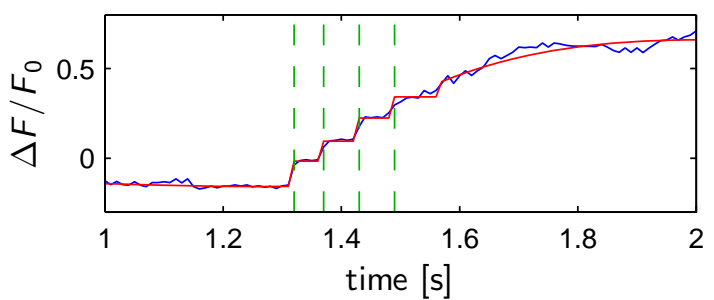

Figure 3.28: Strategies for the detection of single action potentials from the $\mathrm{Ca}^{2+}$ trace. Two algorithms were tested and are presented using odor-evoked $\mathrm{Ca}^{2+}$ traces from two M/T cells. (a) Traces of $\mathrm{Ca}^{2+}$ indicator dyes recorded with a high temporal resolution (here $100 \mathrm{~Hz}$ ) often show step-like shapes, characteristic for occurrence of single action potentials. (b) Calculating local cross-correlation between the $\mathrm{Ca}^{2+}$ trace and a step function results in peaks at the points of presumed APs. By choosing an appropriate threshold (here 0.85) and detecting local maxima in the function yields the candidate time points. (c) Another approach starts from the global fit obtained for the purpose on determination of the response onset. The fit is modified and improved by successively replacing it by steps of constant amplitude. This procedure is repeated as long as the residual between the original trace and the fit is diminished.

recorded $\mathrm{Ca}^{2+}$ traces. Two algorithms were tested on odor-evoked response patterns from data presented here.

The first method is based on a local cross-correlation analysis. To this end, the shape of the $\mathrm{Ca}^{2+}$ trace in response to a single AP is locally approximated by a step 
function (signum function)

$$
s(t)=\left\{\begin{array}{l}
-1, \quad t<0 \\
+1, \quad t \geq 0
\end{array}\right.
$$

To detect $\mathrm{APs}$ from a given $\mathrm{Ca}^{2+}$ trace, a local cross-correlation between the intensity trace $I(t)$ and $s(t)$ is calculated over a window with a width of $(2 n+1)$ time points. For each of the discrete recording times $t_{i}$ a correlation value is obtained:

$$
c\left(t_{i}\right)=\frac{\sum_{\tau=t_{i-n}}^{t_{i+n}} I(\tau) \cdot s\left(\tau-t_{i}\right)}{\sqrt{\|I(t)-\bar{I}\|_{\left[t_{i-n}, t_{i+n}\right]} \cdot\|s(t)-\bar{s}\|_{\left[t_{-n}, t_{+n}\right]}}},
$$

with $\|\cdot\|_{\left[t_{1}, t_{2}\right]}$ being the 2-norm over the interval $\left[t_{1}, t_{2}\right]$. Due to the normalization, $c\left(t_{i}\right)$ is insensitive to the amplitude of the trace in the time window $I(\tau), t_{i-n}<\tau<$ $t_{i+n}$. This is advantageous, since the absolute fluorescent intensity (or the $\Delta F / F_{0}-$ value) depends on parameters that are not relevant for the detection of APs (such as the level of staining). The occurrence of steps in the original trace will be reflected by peaks in the 'correlation-trace' (Fig. 3.28b). The method poses the problem to select an appropriate threshold in order to detect only peaks that are significant. In Figure 3.28b, a threshold of 0.85 was used, but the selection has to be based on statistical criteria (such as thresholding algorithms for the histogram of correlation values), and should be validated by simultaneous electrophysiological recordings.

The second method tested is based on a fitting procedure and does thus not depend on the choice of a threshold. Is starts from the bi-exponential fit that was calculated for the determination of the response onset time in 3.3.2. Assuming that APs are reflected as sharp rising phases in the fluorescent intensity, this fit is modified by consecutively replacing parts of it by individual steps of constant amplitude. After fitting $n$ steps, the function has the form

$$
f_{n}(t)=\left\{\begin{array}{lr}
a_{0} \cdot t+f_{0} & t<t_{0} \\
a_{i} & , t_{i-1}<t<t_{i} \\
a_{0} \cdot t_{\text {on }}^{\mathrm{fit}}+f_{0}+a_{\text {exp }} \cdot \mathrm{e}^{-\left(t-t_{\text {on }}^{\mathrm{fit}}\right) / \tau_{\mathrm{down}}} \cdot\left(1-\mathrm{e}^{\left.-\left(t-t_{\text {on }}^{\mathrm{fit}}\right) / \tau_{\mathrm{up}}\right)}\right), t_{n} & <t,
\end{array}\right.
$$

for $i \in[1, \ldots, n]$. Steps are added as long as the norm of the residual between $I(t)$ and $f_{n}(t)$ is decreased, $\left|I(t)-f_{n}(t)\right|<\left|I(t)-f_{n-1}(t)\right|$. The time points $\left(t_{1}, \ldots, t_{n}\right)$ then indicate the occurrences of APs. 
Testing these procedures using actual recordings of somatic $\mathrm{Ca}^{2+}$ traces, both appear to perform well (based on visual inspection, see Fig. 3.28). However, their performance has to be validated in future experiments by simultaneously recording the electrical activity of the neuron. A reliable determination of APs from population of $\mathrm{M} / \mathrm{T}$ cells will significantly extend the current analysis of spatio-temporal odorevoked response patterns in the OB. 


\section{4}

\section{DISCUSSION}

\subsection{Conclusions}

The coding of odorants in the olfactory system, and in the olfactory bulb (OB) in particular, is an unsolved problem. It is commonly accepted, that both the spatial pattern of activated mitral/tufted $(\mathrm{M} / \mathrm{T})$ cells (the principle neurons of the $\mathrm{OB}$ ), as well as the temporal pattern of their activity are important for the coding of odors. An understanding of the coding principles depends thus on a study of these patterns with a sufficiently high temporal and spatial resolution. Motivated by response times measured in behavioral tasks and by recent findings in other sensory systems, the present study concentrated on the examination of the response onset times across $\mathrm{M} / \mathrm{T}$ cells. Experiments were conducted on larval Xenopus laevis, as it is an approved model system for olfactory research (Czesnik et al., 2003; Manzini et al., 2007a; Scheidweiler et al., 2001). Behaviorally relevant stimuli are known and natural stimulation can be combined with $\mathrm{Ca}^{2+}$ imaging using a nose-brain preparation (Czesnik et al., 2003). The new line-illumination microscope (LIM) enabled the simultaneous measurement of the activity of dozens of individual cells with a temporal resolution of $100 \mathrm{~Hz}$ using $\mathrm{Ca}^{2+}$ indicator dyes. Based on the odor-evoked

$\mathrm{Ca}^{2+}$ time courses, the response onset, i.e. the time of the first spike, could be determined for large numbers of responsive neurons. These times, being recorded simultaneously, were then used to examine the interneuronal dependencies of the activation pattern. It could be shown, that the fluctuations in onset time are correlated between neurons of a slice, which emphasizes the importance of simultaneous recordings. Two measures for the similarity of the population onset times between two odor applications were introduced. These measures are independent to overall 
shifts in onset time and do not rely on time values averaged over trials. First, the newly defined inversion index measures the similarity of the order of response onsets in a neuronal population between two applications. Second, the correlation coefficient measures the linearity of onset times from the responsive neurons between two applications. These measures assess thus different aspects of the pattern of onset times.

By investigating the odor-evoked responses upon stimulation with behaviorally relevant odors in the olfactory bulb of Xenopus laevis tadpoles, the following results were obtained:

1. For a given odor at a fixed concentration, the population onset pattern is highly reproducible, as assessed both by the inversion index and the correlation coefficient.

2. Stimulating with the same odor at different concentration results in a overall decrease of the onset times with increasing concentration for all neurons.

3. The population onset pattern for a given odorant at different concentrations is highly similar, with only a small decrease in similarity with respect to comparing applications using the same concentration as assessed by the two measures.

4. The population onset pattern compared between applications of different odorants on the other hand are very dissimilar and the degree of similarity appears to depend on the pair of odors that are compared.

These experiments focussed on the investigation of one specific aspect of the activity pattern. The presented results can thus not be exhaustive, as there might be other aspects containing odor related information, such as modulation of spontaneous activity, or levels of firing rates across neurons. On the other hand, the approach to include all responsive neurons in the analysis of population onset patterns is very conservative, as there might exist functional subpopulation of cells that are rather independently active. The reproducibility of the onset order across all observed responsive neurons is remarkable and might be even more pronounced for functional neuronal subsets. These observations make the order of response onset a likely 
candidate for an odor-specific code that might be used by higher brain centers, rather than a coincidental epiphenomenon.

Even though the similarity in onset patterns was assessed by two different parameters, it could not be concluded whether the onset order or the durations of the differences in onset times across neurons are more informative about the odor. Both parameters showed a slight dependence on concentration and a strong dependence on odor identity.

In the following, it will be discussed, what further experiments can be done, how the presented results relate to other findings in the olfactory and other sensory systems, and possible strategies for both encoding and decoding of population onset patterns.

Response onset patterns provide a large variety of combinatorial responses, that could be optimized for recognition and differentiation of critical odors by the OB network. The existence of these invariant and specific patterns raises a number of questions about their origin, their relation to properties of the stimulus and possible decoding strategies to extract information from these patterns.

\subsection{Future experiments}

The reported phenomena motivate a number of future experiments:

- Determining the latency patterns for a wider variety of stimuli, it will be possible to infer whether a similarity between two stimuli is reflected in a similarity of the stimulus specific latency patterns. The similarity in stimuli can be either a similar chemical structure for mono-molecular odorants, or a similarity in mixing ratios for more complex odors.

- Experiments investigating the relationship of onset patterns for given odorants and their mixture will uncover whether interactions exist that are more complex than those described in the preliminary results of this thesis (3.3.6). Results of these experiments might be additionally informative about the role of the bulbar network for the manifestation of $\mathrm{M} / \mathrm{T}$ cell onset patterns.

- Similarly to the attempt to quantify the similarity between onset patterns, 
it will be desirable to quantify and compare further aspects of the spatiotemporal activity patterns and their inter-neuronal dependencies, such as spike rate, spike number or inhibitory responses. The different parameters might contain complementary information, might be redundant, or not stimulus specific at all.

- A confirmation of the presented results in other preparations and animal models is desirable. Slice preparations as used for the experiments in this thesis offer great control about the experimental conditions, and have contributed much to today's neuroscientific knowledge. However, whole-brain and in vivo preparations offer systems even closer to the natural state of the nervous system. Currently, staining and imaging techniques are being developed in our laboratory that will allow the investigation of $\mathrm{M} / \mathrm{T}$ cells in the un-cut brain of Xenopus leavis larvae. As the olfactory system is very conserved across animal species, it will be interesting to study population onset patterns in other species, from insects to higher vertebrates.

- Eventually, it will be possible to combine measurements and manipulations at the single-cell level with behavioral experiments. Using the recently discovered channelrhodopsin and their derivatives (Boyden et al., 2005; Nagel et al., 2002), it will be possible to artificially generate spatio-temporal activation patterns in the OB, without a stimulation of the ORNs. By varying these patterns and combining them with behavioral paradigms, such as simple odor discrimination tasks (without actually applying any odor), it will be possible to relate characteristics of the activation patterns to behavioral responses. As the required tools are already available, they will hopefully be combined in the near future to uncover which aspects of the OB code are responsible for odor recognition. 


\subsection{Response latencies in the olfactory and other sensory systems}

\subsubsection{Response latencies in the olfactory system}

A number of studies, using both electrical recordings and imaging of functional dyes, has investigated the properties of odor-evoked response latencies in the olfactory system. While these studies agree in that latencies of $\mathrm{M} / \mathrm{T}$ cell responses are variable, there is no general conclusion as to whether and to what extent these latencies carry relevant information. Further, there has not yet been an approach to quantify similarities in latency patterns between applications of identical or different odorants.

Already in 1977, response latencies were reported based on electrophysiological recordings of mitral cells in the salamander Ambystoma tigrinum (Kauer and Shepherd, 1977). This study reported concentration dependent onset times ranging between 120 and $450 \mathrm{~ms}$ for excitatory responses, though without investigating the odor specificity of these latencies. More recent studies using electrophysiological recordings tried to assess the information contained in the response latencies. These investigations used different approaches for interpreting the acquired data, yielding diverging conclusions. Margrie et al. used the respiration cycle of mice as a temporal reference and showed that the time of the first spike relative to the respiration cycle is strongly correlated to the number of spikes per respiration cycle, both for artificial stimulation of the olfactory nerve and for odor stimulation (Margrie and Schaefer, 2003). They concluded that the time of the first spike is the fastest and most reliable indicator of input strength. This study does however not include an investigation of the odor specificity of the response latencies or of the ensemble patterns of onset times, as it was restricted to a single recording at a time. Bathellier et al. recorded a small number of neurons simultaneously by using tetrodes (a total of 101 neurons from recordings in 13 animals,(Bathellier et al., 2008)). They used a variety of odors and concentrations, and performed classification tests, to assess which feature of the response patterns contains the most odor related information. They concluded that the mean spike rate contains the most information, with la- 
tency and phase of the response showing much higher error rates in the classification task. However, their analysis is based on pooling responses across trials and across animals, which removes temporal dependencies between neurons. A coarse measure like the mean firing rate is insensitive to this averaging procedure, which makes it the most reliable feature of such an analysis. The present study tried to avoid this pitfall by quantifying response patterns on a single trial basis. The imaging approach employed by Spors and Grinvald (2002) is the most similar to the results presented here. While this study reports reproducible patterns of response latencies as measured from postsynaptic glomerular terminals, it does not attempt to quantify the inter-trial degree of similarity, or to investigate the odor specificity of the onset patterns. A quantification of these parameters is crucial for a conclusion about the potential relevance of the observed phenomenon.

An indication that response latencies might play a role for the perception and recognition of odorants came from behavioral tasks in humans (Laing et al., 1994). Using binary mixtures of odorants, it was found that the components are perceived in a temporal order, and that in many cases one component is suppressing the perception of the other component. This suppression could be correlated to the order in which the components where perceived, with the 'faster' component suppressing the 'slower' one. However, the role of 'dominant' and 'suppressed' component as well as their perceived order could be exchanged by introducing a slight temporal delay in the application of the two components (on the order of hundreds of milliseconds). Following the results presented in this thesis, the idea that the components produce overlapping patterns of response latencies, and that the 'earlier' component dominates this pattern and thus the perception is a possible explanation of the neuronal basis of these findings.

\subsubsection{Response latencies in other sensory systems}

Response latencies have been implicated in the stimulus coding in other sensory systems, based both on cellular investigations, and on behavioral studies.

The auditory system The probably most famous example was found in the auditory system. The small temporal difference at which a sound reaches the two 
ears results in the slightly delayed generation of APs in the two sides of the brain. This interaural time difference is used by the nervous system to localize the source of the sound (Carr and Konishi, 1990; Jeffress, 1948). It has been further shown, that first-spike time latencies of auditory cortical neurons contain information about the change of peak pressure in the auditory cortex of cats (Heil, 1997). Additionally, a phenomenon similar to the masking effect reported for the olfactory system (see above, (Laing et al., 1994)) is known from psychoachoustic experiments as the Haas or precedence effect (also known as 'Gesetz der ersten Wellenfront', (Haas, 1949)). When two identical sounds from different sources are played with a slight temporal difference, the observer perceives the sound as coming exclusively from the earlier source.

The visual system In the visual system it was found that the spatial structure of a stimulus is represented in the timing of the first spikes of retinal ganglion cells (Gollisch and Meister, 2008). The stimulus could be recovered more accurately from the latencies of the first spike of the ganglion cells, than from their spike counts. Furthermore, binocular delays have been implicated in the perception of depth (Burr and Ross, 1979), somewhat similar to the sound localization based on interaural time differences. It was further shown that response latencies in the striate cortex of monkeys vary with stimulus contrast and suggested that synchronization based on latencies might contribute to feature binding in the visual system (Gawne et al., 1996). These findings were confirmed and extended more recently (Reich et al., 2001).

The somatosensory system In the somatosensory system, object manipulation tasks showed, that tactile information are used faster by the nervous system than can be explained by using firing rates (Johansson and Birznieks, 2004). However, the activation sequence of different afferents provided the required information fast enough to account for the observed response behavior, implying a role of onset patterns for coding in these tasks. Analysis of spike patterns in the rat barrel cortex revealed that spike timing is more informative about stimulus location than spike number, with the timing of the first spike being particularly informative (Panzeri 
et al., 2001; Petersen et al., 2001, 2002).

\subsection{Onset patterns and properties of the olfactory system}

The observation of odor specific latency patterns in the OB suggests a possible role of these patterns for olfactory coding. While this hypothesis cannot yet be tested directly, it has to be consistent with other properties of the olfactory system.

Latencies of ORN responses Receptor type and concentration dependencies of response latencies in ORNs have been reported across species (Firestein et al., 1993; Friedrich and Korsching, 1997; Getchell and Shepherd, 1978; Gomez and Atema, 1996; Spors et al., 2006), and are in agreement with the present preliminary recordings from ORN terminals in the OB of Xenopus tadpoles. These latencies originate, at least in part, from stimulus integration (Gomez and Atema, 1996), and might be one basis for the latency patterns observed in the OB.

Concentration independence of odor perception It has been shown that recognition and perception of odors is independent over a wide range of concentrations for humans (Gross-Isseroff and Lancet, 1988), rats (Youngentob et al., 1990) and honeybees (Pelz et al., 1997). However, the activity patterns elicited in the OB are not concentration invariant, with large arrays of glomeruli (Johnson and Leon, 2000; Meister and Bonhoeffer, 2001) and M/T cells (Czesnik et al., 2003) being activated with increasing concentration, due to the fact that higher concentrations activate additional receptor types in the olfactory epithelium. Further, the odorevoked time pattern in $\mathrm{M} / \mathrm{T}$ cells were found to be concentration dependent (Wellis et al., 1989). The high degree of similarity of population onset patterns provides a response characteristic that can account for the concentration invariance of the perception. The additional recruitment of $\mathrm{M} / \mathrm{T}$ cells at higher concentrations would not affect the perception, as the cells activated by ORNs with a lower affinity, would be expected to show longer delays. At least one alternative strategy for achieving concentration invariant odor perception has been proposed (Cleland et al., 2007). The model is based on a presumed normalization of $\mathrm{M} / \mathrm{T}$ cell responses, which is 
implemented by the OB network.

Identification of the components of a mixture One property of the olfactory sense (at least in humans) is the limited ability to decompose complex mixtures into their components. Even for small numbers of components (less than five), human subjects were found to perform poorly (Laing and Francis, 1989; Laing and Glemarec, 1992). Given the fact that most natural odorants are complex mixtures, this appears not too surprising, as the olfactory sense seems optimized for recognizing mixtures as undivided entities rather than decomposing them. A coding scheme exploiting response latencies would be in agreement both with the lacking ability to recognize components in mixtures, as well as with an optimization to memorize compound mixtures, as each mixtures (with fixes component-ratios) produces as characteristic population onset pattern. This concept is supported by the recent finding that marginal changes in a mixture are sufficient for an altered perception (Berre et al., 2008). Also the reported suppression of components in a mixture ((Laing et al., 1994), see above) is in accordance with the proposed role of population onset patterns.

Role of sniffing Sniffing is assumed to play a crucial role for odor recognition (Kepecs et al., 2006; Laing, 1986; Scott, 2006; Verhagen et al., 2007). Its assumed relevance is supported by the presence of sniff-like behavior in non-mammalian species ('coughing' in fish (Nevitt, 1991) and antennular flicking in crustaceans (Atema, 1995; Mellon, 1997)). While each sniff is thought to represent a unit of olfactory processing (Kepecs et al., 2006), its precise role is not yet understood. Assuming a role of population onset patterns, sniffing could be assigned at least two concrete roles: to create a temporal reference for the odor onset patterns (similarly to the proposed role of saccades in the visual system (Gollisch and Meister, 2008)), and to improve odor recognition by generating repeated sequence of onset patterns with each sniff, in this way removing uncertainty due to random fluctuations.

Discrimination times for odors of varying similarity It has been reported that the time required to discriminate two binary mixtures depends on the similarity 
of the mixtures (Abraham et al., 2004). This finding is in agreement with a coding scheme using response latencies, as the similarity of two stimuli is reflected in the degree of overlapping responsive $\mathrm{M} / \mathrm{T}$ cell populations. Thus, very different stimuli can be distinguished rapidly, based solely on the initial spatial activity pattern. Highly similar stimuli on the other hand depend on the temporal onset patterns, and possibly on the late activation of weakly activated cells that might be specific of either stimulus.

Structure and role of olfactory glomeruli The olfactory glomeruli are a unique structure of the olfactory system. It is assumed that they serve to improve the signal-to-noise ratio by integrating responses from a large number of receptor neurons. Considering the idea of coding with response latencies, they could have a more distinct role, namely the generation of a reproducible onset time even for low concentration of odorant. It is known that the response latency of ORNs varies greatly, especially for low odor concentrations (Gomez and Atema, 1996). By integrating the responses from many ORNs of the same type, as realized in the olfactory glomeruli, this variability could be greatly reduced, resulting in reproducible onset times even for very low concentrations.

Spontaneous activity and inhibitory responses in the $\mathrm{OB} \mathrm{M} / \mathrm{T}$ cells are known to be occasionally spontaneously active at low spiking rates $(0.3 \mathrm{~Hz}$ in salamander (Kauer and Shepherd, 1977), $0.5 \mathrm{~Hz}$ in frog (Doeving, 1964) and $0.6 \mathrm{~Hz}$ in mice (Margrie et al., 2002)). While it was suggested that the spontaneous activity in receptor neurons plays a role for the establishment and maintenance of an olfactory sensory map (Yu et al., 2004), it is not clear whether the spontaneous activity of $\mathrm{M} / \mathrm{T}$ cells (or its modulation) contributes to the olfactory code. Further, $\mathrm{M} / \mathrm{T}$ cells are known to be inhibited in response to some odors. It is not clear whether the observed suppression of spontaneous activity is part of the olfactory code used by higher centers, or whether it is due to lateral inhibition that is only relevant in the sense of a not-activated $\mathrm{M} / \mathrm{T}$ cell. The sparseness and low firing rate of the spontaneous activity do not support a crucial role of its suppression for fast coding. In particular, the onset time of an inhibitory response is not identifiable accurately 
(Kauer and Shepherd, 1977), making these responses unsuitable for a coding scheme based on response onset times. In insects, spontaneous activity of projection neurons (the equivalent of vertebrate $\mathrm{M} / \mathrm{T}$ cells) has been implicated in the formation of memory, based on the comparison of pre- and post-stimulus spontaneous activity (Galán et al., 2006).

\subsection{Decoding of response latency patterns}

The proposition of a coding scheme immediately raises the question about potential read-out mechanisms that might be employed by higher brain areas, i.e. mechanisms for decoding the stimulus under the proposed coding scheme. Many of the proposed coding schemes suggested for the olfactory system relied on a global reference signal, the information being contained in the timing of spikes relative to this reference. In most cases, this global reference was suggested to be the local field potential oscillations in the $\gamma$ range (Hopfield, 1995; Laurent et al., 2001), but also subthreshold $\theta$-oscillations in $\mathrm{M} / \mathrm{T}$ cells which are locked to the breathing cycle were suggested (Margrie and Schaefer, 2003). While this is a possible coding strategy, there is at least one report arguing against an essential role of these oscillations for olfactory coding. It was reported, that young rats (postnatal day 7) lack the local field potential $\gamma$-oscillations, but show no significant difference in either the receptive fields of $\mathrm{M} / \mathrm{T}$ cells, nor in behavioral odor discrimination. A role of oscillations coupled to the breathing cycle appears tempting, as the frequency of these oscillations match the rather slow dynamics of the OB. However, while breathing-like behavior has been described for some fish (Nevitt, 1991) and crustaceans (Atema, 1995; Mellon, 1997), it is probably not a feature common for all animals or even vertebrates. This does not exclude that sniffing might help to increase accuracy and sensitivity, but makes it an unlikely candidate for a required general aspect of olfactory coding.

One reason that global oscillations have been favored by scientists might originate from the inability to synchronously record the activity of a large number of neurons. The observed oscillations were a convenient way to temporally relate successive recording, by aligning them to the phase of the oscillations. Such a global reference is however not an essential part of a multi-neuron coding scheme. Instead, 
the spike times across neurons might carry information that can be read out by higher centers without relying on a global reference frame. An example for such a coding with inter-neuronal latencies is the sound localization based on inter-aural time differences mentioned earlier (Carr and Konishi, 1990; Jeffress, 1948). The time difference between spikes from the two sides of the brain are read out - decoded - using axonal delays. A generalized version of this mechanisms could be used by higher olfactory centers. The time delay associated with certain odors would have to be learned by such a system. In fact, it has been noted by Hopfield that the piriform cortex, one of the targets of $\mathrm{OB}$ output, is a suitable structure for learning and decoding inter-neuronal time delays (Hopfield, 1995): 'The simplest scheme is to have a multiplicity of time delays, direct or indirect, available via different synapses. This would be easily done in a structure like the piriform cortex, where the recurrent collaterals allow many possible indirect paths of different time delay between two cells. In such a structure the correct time delays would merely need to be selected by strengthening appropriate synapses using a Hebb-like rule.' Another biologically plausible mechanism for decoding spatio-temporal spike patterns has been introduced recently as the Tempotron by Gütig and Sompolinsky (2006). They demonstrated that a learning rule based on information contained in the spatio-temporal structure of spike patterns, rather than in mean firing rates, is capable of distinguishing large numbers of input patterns. Additionally, it was shown that the learning capacity of the tempotron exceeds the capacity of comparable decoders based on mean firing rates, such as a single-layer perceptron (Gütig and Sompolinsky, 2006; Minsky and Papert, 1969) .

The presented results suggest a role of $\mathrm{M} / \mathrm{T}$ cell response onset patterns for olfactory coding. A coding scheme relying at least in parts on these ensemble patterns appears consistent with the properties of the olfactory system, could be decoded by higher brain centers and is supported by similar observations in other sensory systems. 


\section{5}

\section{SUMMARY}

This thesis aimed at investigating coding principles of the olfactory system by developing novel experimental and analytical tools and applying them to specific biological questions. The olfactory bulb $(\mathrm{OB})$ is the only central processing station of the vertebrate olfactory system. It is assumed that odors are represented by a spatio-temporal code in the OB, with both the identity of the activated neurons and the temporal sequence of their activity patterns being stimulus-relevant parameters. An adequate investigation of these coding principles thus relies on the simultaneous recording of a large number of cells with a high temporal resolution. To date, few studies have been based on recordings of this type, and none of these has attempted to investigate and quantify aspects of inter-neuronal dependencies in the odor-evoked activity patterns.

First, a fast confocal microscope was designed, built and characterized. By scanning the sample with a line instead of a point, and by using a fast sensor, high frame rates can be achieved. The aperture provided by the pixel width of the CCD camera provides optical sectioning capabilities. The microscope was optimized for light efficiency in the emission pathway, as biological specimens demand both low levels of illumination and repeated trials of a given experiment. The microscope was characterized with respect to its spatial and temporal resolution, as these are key parameters for investigating neuronal networks. It was shown that the spatial resolution is adequate for the observation of single neurons, with high magnification objectives providing sufficient resolution even for small neuronal processes. The temporal resolution depends on the pixel dimension of the acquired frame, ranging from $50 \mathrm{~Hz}(512 \times 512$ pixels $)$ to $800 \mathrm{~Hz}(512 \times 32$ pixels $)$ of maximum frame rates. The microscope was thus shown to be a suitable tool for investigating the 
spatio-temporal activity patterns of neurons in the OB.

As a first application, a novel method for the visualization of neuronal morphology based on functional imaging data was demonstrated. Time series of image stacks of spontaneous activity, visualized using $\mathrm{Ca}^{2+}$ indicator dyes, were acquired with subsecond resolution. By exploiting the diverse temporal activity patterns as a means of intrinsic contrast, the individual neurons' dendritic morphology could be visualized. The result of this novel method, termed activity correlation imaging, is a high-contrast multi-color visualization of the neuronal network, as demonstrated on the mitral/tufted cells of the Xenopus larvae OB. Yielding both functional and structural information about neuronal populations, this method opens up unprecedented possibilities for the investigation of neuronal networks.

Various aspects of the odor-evoked activity patterns in the mitral/tufted cells have been suggested as being relevant for olfactory coding. Motivated by findings in other sensory systems, and by the short time scales set by various olfactory discrimination tasks in behavioral experiments, the timing of the onset of odor-evoked responses was investigated. The simultaneous recording of dozens of neurons enables the investigation of inter-neuronal dependencies in the odor-evoked responses, which would be obscured by inter-trial variability in successive single-cell recordings. To this end, nose-brain preparations of Xenopus laevis larvae were stained with $\mathrm{Ca}^{2+}$ indicator dye, stimulated with natural odorants and imaged with a high temporal resolution $(100 \mathrm{~Hz})$. A novel measure, named the inversion index, was introduced to quantify the similarity in the order of response onsets between pairs of stimulus applications. Using this measure, it was shown that these patterns are highly reproducible between applications of a given odor, both for mono-molecular odorants and for complex mixtures. Additionally, the onset order was found to be highly informative about odor identity, while it only relies weakly on odor concentration. These observations make the onset pattern of $\mathrm{M} / \mathrm{T}$ cell ensembles a promising candidate for a stimulus-relevant feature of the code implemented in the OB. The thesis is concluded by relating the reported findings to other properties of the olfactory system and by discussing possible decoding strategies for the observed response onset patterns. 


\section{BIBLIOGRAPHY}

Abbe, E.: Beitrage zur Theorie des Mikroskops und der mikroskopischen Wahrnehmung. Arch. Mikrosk. Anat. 9: 413 - 420, 1873.

Abraham, N.M., Spors, H., Carleton, A., Margrie, T.W., Kuner, T., and Schaefer, A.T.: Maintaining accuracy at the expense of speed: stimulus similarity defines odor discrimination time in mice. Neuron 44 (5): 865 - 876, 2004.

Ache, B.W. and Young, J.M.: Olfaction: diverse species, conserved principles. Neuron 48 (3): $417-430,2005$.

Adrian, E.D.: Olfactory reactions in the brain of the hedgehog. J Physiol 100 (4): $459-473,1942$.

Aroniadou-Anderjaska, V., Ennis, M., and Shipley, M.T.: Glomerular synaptic responses to olfactory nerve input in rat olfactory bulb slices. Neuroscience $\mathbf{7 9}(2)$ : $425-434,1997$.

Atema, J.: Chemical signals in the marine environment: dispersal, detection, and temporal signal analysis. Proc Natl Acad Sci U S A 92 (1): 62-66, 1995.

Aungst, J.L., Heyward, P.M., Puche, A.C., Karnup, S.V., Hayar, A., Szabo, G., and Shipley, M.T.: Centre-surround inhibition among olfactory bulb glomeruli. Nature 426 (6967): 623 - 629, 2003.

Bardoni, R., Magherini, P.C., and Belluzzi, O.: Excitatory synapses in the glomerular triad of frog olfactory bulb in vitro. Neuroreport 7 (11): 1851 - 1855, 1996. 
Bathellier, B., Buhl, D.L., Accolla, R., and Carleton, A.: Dynamic ensemble odor coding in the mammalian olfactory bulb: sensory information at different timescales. Neuron 57 (4): 586 - 598, 2008.

Beichelt, F.E. and Montgomery, D.C. (Editors): Teubner-Taschenbuch der Stochastik. Teubner, Wiesbaden, $1^{\text {st }}$ edition, 2003.

Berkowicz, D.A., Trombley, P.Q., and Shepherd, G.M.: Evidence for glutamate as the olfactory receptor cell neurotransmitter. J Neurophysiol 71 (6): 2557 - 2561, 1994.

Berre, E.L., BÃlno, N., Ishii, A., Chabanet, C., Etiấlvant, P., and ThomasDanguin, T.: Just noticeable differences in component concentrations modify the odor quality of a blending mixture. Chem Senses 33 (4): 389-395, 2008.

Betzig, E., Patterson, G.H., Sougrat, R., Lindwasser, O.W., Olenych, S., Bonifacino, J.S., Davidson, M.W., Lippincott-Schwartz, J., and Hess, H.F.: Imaging intracellular fluorescent proteins at nanometer resolution. Science 313 (5793): 1642 $1645,2006$.

Bischofberger, J. and Jonas, P.: Action potential propagation into the presynaptic dendrites of rat mitral cells. J Physiol 504: 359 - 365, 1997.

Bisulco, S. and Slotnick, B.: Olfactory discrimination of short chain fatty acids in rats with large bilateral lesions of the olfactory bulbs. Chem Senses 28 (5): 361 $-370,2003$.

Boos, D.D.: Introduction to the Bootstrap World. Statistical Science 18 (2): 168 $174,2003$.

Boyden, E.S., Zhang, F., Bamberg, E., Nagel, G., and Deisseroth, K.: Millisecondtimescale, genetically targeted optical control of neural activity. Nat Neurosci 8 (9): 1263-1268, 2005.

Buck, L. and Axel, R.: A novel multigene family may encode odorant receptors: a molecular basis for odor recognition. Cell 65 (1): 175 - 187, 1991. 
Buck, L.B.: Information coding in the vertebrate olfactory system. Annu Rev Neurosci 19: 517 - 544, 1996.

Burr, D.C. and Ross, J.: How does binocular delay give information about depth? Vision Res 19 (5): 523 - 532, 1979.

Byrd, C.A. and Brunjes, P.C.: Organization of the olfactory system in the adult zebrafish: histological, immunohistochemical, and quantitative analysis. J Comp Neurol 358 (2): $247-259,1995$.

Carr, C.E. and Konishi, M.: A circuit for detection of interaural time differences in the brain stem of the barn owl. J Neurosci 10 (10): 3227 - 3246, 1990.

Carr, W.: Sensory Biology of Aquatic Animals. Springer, New York, 1988.

Charpak, S., Mertz, J., Beaurepaire, E., Moreaux, L., and Delaney, K.: Odor-evoked calcium signals in dendrites of rat mitral cells. Proc Natl Acad Sci USA 98 (3): $1230-1234,2001$.

Christie, J.M., Bark, C., Hormuzdi, S.G., Helbig, I., Monyer, H., and Westbrook, G.L.: Connexin36 mediates spike synchrony in olfactory bulb glomeruli. Neuron 46 (5): $761-772,2005$.

Cleland, T.A., Johnson, B.A., Leon, M., and Linster, C.: Relational representation in the olfactory system. Proc Natl Acad Sci USA 104 (6): 1953 - 1958, 2007.

Czesnik, D., Nezlin, L., Rabba, J., Müller, B., and Schild, D.: Noradrenergic modulation of calcium currents and synaptic transmission in the olfactory bulb of Xenopus laevis tadpoles. Eur J Neurosci 13 (6): 1093 - 1100, 2001.

Czesnik, D., Rössler, W., Kirchner, F., Gennerich, A., and Schild, D.: Neuronal representation of odourants in the olfactory bulb of Xenopus laevis tadpoles. Eur J Neurosci 17 (1): 113 - 118, 2003.

Doeving, K.B.: Studies of the relation between the frog's electro-olfactogram (EOG) and single unit activity in the olfactory bulb. Acta Physiol Scand 60: 150 - 163, 1964. 
Duchamp-Viret, P., Chaput, M.A., and Duchamp, A.: Odor response properties of rat olfactory receptor neurons. Science 284 (5423): 2171 - 2174, 1999.

Edwards, F.A., Konnerth, A., Sakmann, B., and Takahashi, T.: A thin slice preparation for patch clamp recordings from neurones of the mammalian central nervous system. Pflugers Arch 414 (5): 600 - 612, 1989.

Efron, B.: Bootstrap methods: Another look at the jacknife. The Annals of Statistics 7 (1): $1-26,1979$.

Eisthen, H.L.: Why are olfactory systems of different animals so similar? Brain Behav Evol 59 (5-6): 273 - 293, 2002.

Engelmann, R.: Faster than real-time: confocal linescan systems provide ideal conditions for millisecond-resolution physiological imaging (Advertising Feature). Nat Methods 3, 2006.

Ennis, M., Zimmer, L.A., and Shipley, M.T.: Olfactory nerve stimulation activates rat mitral cells via NMDA and non-NMDA receptors in vitro. Neuroreport 7 (5): $989-992,1996$.

Fantana, A.L., Soucy, E.R., and Meister, M.: Rat olfactory bulb mitral cells receive sparse glomerular inputs. Neuron 59 (5): 802 - 814, 2008.

Fecteau, J. and Milgram, N.W.: The ability to smell remains intact, but does not recover, after olfactory bulb lesions. Int J Neurosci 108 (1-2): 11 - 20, 2001.

Ferrer, R.P. and Zimmer, R.K.: The scent of danger: arginine as an olfactory cue of reduced predation risk. J Exp Biol 210 (Pt 10): 1768 - 1775, 2007.

Firestein, S., Picco, C., and Menini, A.: The relation between stimulus and response in olfactory receptor cells of the tiger salamander. J Physiol 468: 1-10, 1993.

Fletcher, M.L., Smith, A.M., Best, A.R., and Wilson, D.A.: High-frequency oscillations are not necessary for simple olfactory discriminations in young rats. $J$ Neurosci 25 (4): $792-798,2005$.

Freeman, W.J.: The physiology of perception. Sci Am 264 (2): 78 - 85, 1991. 
Freeman, W.J.: Neural networks and chaos. J Theor Biol 171 (1): 13 - 18, 1994.

Friedrich, R.W. and Korsching, S.I.: Combinatorial and chemotopic odorant coding in the zebrafish olfactory bulb visualized by optical imaging. Neuron 18 (5): 737 $-752,1997$.

Friedrich, R.W. and Korsching, S.I.: Chemotopic, combinatorial, and noncombinatorial odorant representations in the olfactory bulb revealed using a voltage-sensitive axon tracer. J Neurosci 18 (23): 9977 - 9988, 1998.

Friedrich, R.W. and Laurent, G.: Dynamic optimization of odor representations by slow temporal patterning of mitral cell activity. Science 291 (5505): 889 - 894, 2001.

Friedrich, R.W.: Mechanisms of odor discrimination: neurophysiological and behavioral approaches. Trends Neurosci 29 (1): 40 - 47, 2006.

Friedrich, R.W., Habermann, C.J., and Laurent, G.: Multiplexing using synchrony in the zebrafish olfactory bulb. Nat Neurosci 7 (8): $862-871,2004$.

Galán, R.F., Weidert, M., Menzel, R., Herz, A.V.M., and Galizia, C.G.: Sensory memory for odors is encoded in spontaneous correlated activity between olfactory glomeruli. Neural Comput 18 (1): 10 - 25, 2006.

Garaschuk, O., Milos, R.I., and Konnerth, A.: Targeted bulk-loading of fluorescent indicators for two-photon brain imaging in vivo. Nat Protoc 1 (1): 380 - 386, 2006.

Gawne, T.J., Kjaer, T.W., and Richmond, B.J.: Latency: another potential code for feature binding in striate cortex. J Neurophysiol 76 (2): 1356 - 1360, 1996.

Gennerich, A.: Intrazelluläre Fluoreszenz-Korrelations-Spektroskopie: Analyse molekularer Eigenschaften in räumlich eingeschränkten Diffusionssystemen. Master's thesis, Drittes Physikalisches Institut der Georg-August-Universität, Universität Göttingen, 1999.

Getchell, T.V.: Analysis of intracellular recordings from salamander olfactory epithelium. Brain Res 123 (2): 275 - 286, 1977. 
Getchell, T.V. and Shepherd, G.M.: Responses of olfactory receptor cells to step pulses of odour at different concentrations in the salamander. J Physiol 282: 521 $-540,1978$.

Giraudet, P., Berthommier, F., and Chaput, M.: Mitral cell temporal response patterns evoked by odor mixtures in the rat olfactory bulb. J Neurophysiol 88 (2): $829-838,2002$.

Göbel, W., Kampa, B.M., and Helmchen, F.: Imaging cellular network dynamics in three dimensions using fast 3D laser scanning. Nat Methods 4 (1): 73 - 79, 2007.

Gollisch, T. and Meister, M.: Rapid neural coding in the retina with relative spike latencies. Science 319 (5866): 1108 - 1111, 2008.

Gomez, G. and Atema, J.: Temporal resolution in olfaction: stimulus integration time of lobster chemoreceptor cells. J Exp Biol 199 (Pt 8): 1771 - 1779, 1996.

Graziadei, P.P.C.: Handbook of sensory physiology, volume 4, chapter 'The olfactory mucosa of vertebrates'. New York: Springer, 1971.

Gross-Isseroff, R. and Lancet, D.: Concentration-dependent changes of perceived odor quality. Chem Senses 13 (2): 191 - 204, 1988.

Gütig, R. and Sompolinsky, H.: The tempotron: a neuron that learns spike timingbased decisions. Nat Neurosci 9 (3): 420-428, 2006.

Haas, H.: Über den Einfluss eines Einfachechos auf die Hörsamkeit von Sprache. Ph.D. thesis, University of Göttingen, 1949.

Hansson, B.S. and Anton, S.: Function and morphology of the antennal lobe: new developments. Annu Rev Entomol 45: 203 - 231, 2000.

Hartline, H.K.: Visual receptors and retinal interaction. Science 164 (877): 270 278, 1969.

Hassenklöver, T., Kurtanska, S., Bartoszek, I., Junek, S., Schild, D., and Manzini, I.: Nucleotide-induced $\mathrm{Ca}^{2+}$ signaling in sustentacular supporting cells of the olfactory epithelium. Glia 56 (15): 1614 - 1624, 2008. 
Heil, P.: Auditory cortical onset responses revisited. i. first-spike timing. J Neurophysiol 77 (5): 2616-2641, 1997.

Hell, S.W.: Toward fluorescence nanoscopy. Nat Biotechnol 21 (11): 1347 - 1355, 2003.

Hildebrand, J.G. and Shepherd, G.M.: Mechanisms of olfactory discrimination: converging evidence for common principles across phyla. Annu Rev Neurosci 20: 595 $-631,1997$.

Holekamp, T.F., Turaga, D., and Holy, T.E.: Fast three-dimensional fluorescence imaging of activity in neural populations by objective-coupled planar illumination microscopy. Neuron 57 (5): $661-672,2008$.

Hopfield, J.J.: Olfactory computation and object perception. Proc Natl Acad Sci U $S A 88$ (15): 6462-6466, 1991.

Hopfield, J.J.: Pattern recognition computation using action potential timing for stimulus representation. Nature 376 (6535): 33 - 36, 1995.

Hopfield, J.J.: Odor space and olfactory processing: collective algorithms and neural implementation. Proc Natl Acad Sci U S A 96 (22): 12.506-12.511, 1999.

Hopfield, J.J. and Brody, C.D.: Learning rules and network repair in spike-timingbased computation networks. Proc Natl Acad Sci U S A 101 (1): 337-342, 2004.

Ikegaya, Y., Aaron, G., Cossart, R., Aronov, D., Lampl, I., Ferster, D., and Yuste, R.: Synfire chains and cortical songs: temporal modules of cortical activity. Science 304 (5670): 559 - 564, 2004.

Im, K.B., Han, S., Park, H., Kim, D., and Kim, B.M.: Simple high-speed confocal line-scanning microscope. Opt. Express 13 (13): 5151 - 5156, 2005.

Isaacson, J.S.: Glutamate spillover mediates excitatory transmission in the rat olfactory bulb. Neuron 23 (2): 377 - 384, 1999.

Isaacson, J.S. and Strowbridge, B.W.: Olfactory reciprocal synapses: dendritic signaling in the CNS. Neuron 20 (4): 749 - 761, 1998. 
Jahr, C.E. and Nicoll, R.A.: An intracellular analysis of dendrodendritic inhibition in the turtle in vitro olfactory bulb. J Physiol 326: 213 - 234, 1982.

Jeffress, L.: A place theory of sound localization. Journal of Comparative and Physiological Psychology 41: 35 - 39, 1948.

Johansson, R.S. and Birznieks, I.: First spikes in ensembles of human tactile afferents code complex spatial fingertip events. Nat Neurosci 7 (2): 170-177, 2004.

Johnson, B.A. and Leon, M.: Modular representations of odorants in the glomerular layer of the rat olfactory bulb and the effects of stimulus concentration. J Comp Neurol 422 (4): 496 - 509, 2000.

Johnson, B.A., Woo, C.C., Hingco, E.E., Pham, K.L., and Leon, M.: Multidimensional chemotopic responses to n-aliphatic acid odorants in the rat olfactory bulb. J Comp Neurol 409 (4): 529 - 548, 1999.

Kajiya, K., Inaki, K., Tanaka, M., Haga, T., Kataoka, H., and Touhara, K.: Molecular bases of odor discrimination: Reconstitution of olfactory receptors that recognize overlapping sets of odorants. J Neurosci 21 (16): 6018-6025, 2001.

Kandel, E.R., Schwartz, J.H., and Jessell, T.M. (Editors): Principles of neural science. McGraw-Hill, $4^{\text {th }}$ edition, 2000.

Kang, J. and Caprio, J.: In vivo responses of single olfactory receptor neurons of channel catfish to binary mixtures of amino acids. J Neurophysiol 77 (1): $1-8$, 1997.

Kauer, J.S.: Contributions of topography and parallel processing to odor coding in the vertebrate olfactory pathway. Trends Neurosci 14 (2): 79 - 85, 1991.

Kauer, J.S. and Shepherd, G.M.: Analysis of the onset phase of olfactory bulb unit responses to odour pulses in the salamander. J Physiol 272 (2): 495 - 516, 1977.

Kepecs, A., Uchida, N., and Mainen, Z.F.: The sniff as a unit of olfactory processing. Chem Senses 31 (2): 167-179, 2006. 
Khan, A.G., Thattai, M., and Bhalla, U.S.: Odor representations in the rat olfactory bulb change smoothly with morphing stimuli. Neuron 57 (4): 571 - 585, 2008.

Kiesecker, J., D.P., C., and Blaustein, A.: The use of chemical cues in predator recognition by western toad tadpoles. Animal Behaviour 52 (6): 1237 - 1245, 1996.

Kiseleva, E.I.: Sensitivity of the rana temporaria tadpole chemoreceptor system to amino acids. Zh Evol Biokhim Fiziol 19 (1): 99 - 102, 1983.

Kiseleva, E.I.: Natural amino acids as effective stimuli evoking chemoreceptordirected behavior in anuran tadpoles. Zh Obshch Biol 56 (3): 329 - 345, 1995.

Knight, M.M., Roberts, S.R., Lee, D.A., and Bader, D.L.: Live cell imaging using confocal microscopy induces intracellular calcium transients and cell death. Am J Physiol Cell Physiol 284 (4): C1083 - C1089, 2003.

Korsching, S.I., Argo, S., Campenhausen, H., Friedrich, R.W., Rummrich, A., and Weth, F.: Olfaction in zebrafish: what does a tiny teleost tell us? Semin Cell Dev Biol 8 (2): $181-187,1997$.

Kuwahara, M., Hachimura, K., Eiho, S., and Kinoshita, M.: Digital Processing of Biomedical Images, chapter Processing of RI-angiocardiographic images, pages 187 - 202. University of Tokyo Press, 1976.

Laing, D.G.: Identification of single dissimilar odors is achieved by humans with a single sniff. Physiol Behav 37 (1): 163 - 170, 1986.

Laing, D.G., Eddy, A., Francis, G.W., and Stephens, L.: Evidence for the temporal processing of odor mixtures in humans. Brain Res 651 (1-2): 317 - 328, 1994.

Laing, D.G. and Francis, G.W.: The capacity of humans to identify odors in mixtures. Physiol Behav 46 (5): 809-814, 1989.

Laing, D.G. and Glemarec, A.: Selective attention and the perceptual analysis of odor mixtures. Physiol Behav 52 (6): 1047-1053, 1992. 
Laurent, G.: A systems perspective on early olfactory coding. Science 286 (5440): $723-728,1999$.

Laurent, G., Stopfer, M., Friedrich, R.W., Rabinovich, M.I., Volkovskii, A., and Abarbanel, H.D.: Odor encoding as an active, dynamical process: experiments, computation, and theory. Annu Rev Neurosci 24: 263 - 297, 2001.

Laurent, G.: Olfactory network dynamics and the coding of multidimensional signals. Nat Rev Neurosci 3 (11): 884 - 895, 2002.

Lin, B.J., Chen, T.W., and Schild, D.: Cell type-specific relationships between spiking and $\left[\mathrm{Ca}^{2+}\right]_{\mathrm{i}}$ in neurons of the Xenopus tadpole olfactory bulb. J Physiol $582(1): 163-175,2007$.

Livet, J., Weissman, T.A., Kang, H., Draft, R.W., Lu, J., Bennis, R.A., Sanes, J.R., and Lichtman, J.W.: Transgenic strategies for combinatorial expression of fluorescent proteins in the nervous system. Nature 450 (7166): 56 - 62, 2007.

Lledo, P.M., Gheusi, G., and Vincent, J.D.: Information processing in the mammalian olfactory system. Physiol Rev 85 (1): 281 - 317, 2005.

Malnic, B., Hirono, J., Sato, T., and Buck, L.B.: Combinatorial receptor codes for odors. Cell 96 (5): $713-723,1999$.

Manzini, I.: Diversity of transduction mechanisms in receptor neurons of the main olfactory epithelium in Xenopus laevis tadpoles. Ph.D. thesis, International MaxPlanck Research School for Neurosciences, 2002.

Manzini, I., Brase, C., Chen, T.W., and Schild, D.: Response profiles to amino acid odorants of olfactory glomeruli in larval Xenopus laevis. J Physiol 581 (Pt 2): $567-579,2007$ a.

Manzini, I., Heermann, S., Czesnik, D., Brase, C., Schild, D., and Rössler, W.: Presynaptic protein distribution and odour mapping in glomeruli of the olfactory bulb of Xenopus laevis tadpoles. Eur J Neurosci 26 (4): 925 - 934, $2007 \mathrm{~b}$. 
Manzini, I., Peters, F., and Schild, D.: Odorant responses of Xenopus laevis tadpole olfactory neurons: a comparison between preparations. J Neurosci Methods 121 (2): 159 - 167, 2002.

Manzini, I. and Schild, D.: cAMP-independent olfactory transduction of amino acids in Xenopus laevis tadpoles. J Physiol 551 (Pt 1): 115 - 123, 2003a.

Manzini, I. and Schild, D.: Multidrug resistance transporters in the olfactory receptor neurons of Xenopus laevis tadpoles. J Physiol 546 (Pt 2): 375 - 385, 2003b.

Manzini, I. and Schild, D.: Classes and narrowing selectivity of olfactory receptor neurons of Xenopus laevis tadpoles. J Gen Physiol 123 (2): 99 - 107, 2004.

Manzini, I., Schweer, T.S., and Schild, D.: Improved fluorescent (calcium indicator) dye uptake in brain slices by blocking multidrug resistance transporters. $J$ Neurosci Methods 167 (2): 140 - 147, 2008.

Mao, B.Q., Hamzei-Sichani, F., Aronov, D., Froemke, R.C., and Yuste, R.: Dynamics of spontaneous activity in neocortical slices. Neuron 32 (5): 883-898, 2001.

Margrie, T.W., Brecht, M., and Sakmann, B.: In vivo, low-resistance, wholecell recordings from neurons in the anaesthetized and awake mammalian brain. Pflugers Arch 444 (4): 491-498, 2002.

Margrie, T.W. and Schaefer, A.T.: Theta oscillation coupled spike latencies yield computational vigour in a mammalian sensory system. $J$ Physiol 546 (Pt 2): 363 $-374,2003$.

Masters, B.R. and Thaer, A.A.: In vivo human corneal confocal microscopy of identical fields of subepithelial nerve plexus, basal epithelial, and wing cells at different times. Microsc Res Tech 29 (5): 350 - 356, 1994.

Maurice, D.M.: A scanning slit optical microscope. Invest Ophthalmol 13 (12): 1033-1037, 1974. 
McNamara, A.M., Magidson, P.D., and Linster, C.: Binary mixture perception is affected by concentration of odor components. Behav Neurosci 121 (5): 1132 1136, 2007. Noch nicht gelesen.

Meister, M. and Bonhoeffer, T.: Tuning and topography in an odor map on the rat olfactory bulb. J Neurosci 21 (4): 1351 - 1360, 2001.

Mellon, D.J.: Physiological characterization of antennular flicking reflexes in the crayfish. J. Comp. Physiol. A 180 (5): 553 - 565, 1997.

Minsky, M.: U.S. Patent \#3013467, Microscopy apparatus. 1957.

Minsky, M.: Memoir on inventing the confocal scanning microscope. Scanning 10: $128-138,1988$.

Minsky, M. and Papert, S.: Perceptrons. MIT Press, Cambridge, MA, 1969.

Mombaerts, P.: Seven-transmembrane proteins as odorant and chemosensory receptors. Science 286 (5440): $707-711,1999$.

Mombaerts, P.: Odorant receptor gene choice in olfactory sensory neurons: the one receptor-one neuron hypothesis revisited. Curr Opin Neurobiol 14 (1): 31 - 36, 2004.

Mori, K., Kishi, K., and Ojima, H.: Distribution of dendrites of mitral, displaced mitral, tufted, and granule cells in the rabbit olfactory bulb. J Comp Neurol 219 (3): 339 - 355, 1983.

Nagayama, S., Zeng, S., Xiong, W., Fletcher, M.L., Masurkar, A.V., Davis, D.J., Pieribone, V.A., and Chen, W.R.: In vivo simultaneous tracing and ca $(2+)$ imaging of local neuronal circuits. Neuron 53 (6): 789 - 803, 2007.

Nagel, G., Ollig, D., Fuhrmann, M., Kateriya, S., Musti, A.M., Bamberg, E., and Hegemann, P.: Channelrhodopsin-1: a light-gated proton channel in green algae. Science 296 (5577): 2395-2398, 2002.

Nevitt, G.A.: Do fish sniff? A new mechanism of olfactory sampling in pleuronectid flounders. J Exp Biol 157: 1-18, 1991. 
Nezlin, L.P. and Schild, D.: Structure of the olfactory bulb in tadpoles of Xenopus laevis. Cell Tissue Res 302 (1): 21 - 29, 2000.

Nezlin, L.P., Heermann, S., Schild, D., and Rössler, W.: Organization of glomeruli in the main olfactory bulb of Xenopus laevis tadpoles. J Comp Neurol 464 (3): $257-268,2003$.

Nezlin, L.P. and Schild, D.: Individual olfactory sensory neurons project into more than one glomerulus in Xenopus laevis tadpole olfactory bulb. J Comp Neurol 481 (3): $233-239,2005$.

Nicoll, R.A. and Jahr, C.E.: Self-excitation of olfactory bulb neurones. Nature 296 (5856): $441-444,1982$.

Nieuwkoop, P. and Faber, J. (Editors): Normal table of Xenopus laevis (Daudin). Garland Publishing Inc, New York, $2^{\text {nd }}$ edition, 1967.

Niimura, Y. and Nei, M.: Evolutionary dynamics of olfactory and other chemosensory receptor genes in vertebrates. J Hum Genet 51 (6): 505 - 517, 2006.

Ohki, K., Chung, S., Ch'ng, Y.H., Kara, P., and Reid, R.C.: Functional imaging with cellular resolution reveals precise micro-architecture in visual cortex. Nature 433 (7026): $597-603,2005$.

Ohki, K., Chung, S., Kara, P., HÃijbener, M., Bonhoeffer, T., and Reid, R.C.: Highly ordered arrangement of single neurons in orientation pinwheels. Nature 442 (7105): 925 - 928, 2006.

Okano, M. and Takagi, S.F.: Secretion and electrogenesis of the supporting cell in the olfactory epithelium. J Physiol 242 (2): 353 - 370, 1974.

Orbach, H.S., Cohen, L.B., and Grinvald, A.: Optical mapping of electrical activity in rat somatosensory and visual cortex. J Neurosci 5 (7): 1886 - 1895, 1985.

Orona, E., Rainer, E.C., and Scott, J.W.: Dendritic and axonal organization of mitral and tufted cells in the rat olfactory bulb. J Comp Neurol 226 (3): $346-$ 356,1984 . 
Panzeri, S., Petersen, R.S., Schultz, S.R., Lebedev, M., and Diamond, M.E.: The role of spike timing in the coding of stimulus location in rat somatosensory cortex. Neuron 29 (3): $769-777,2001$.

Paternostro, M.A., Reyher, C.K., and Brunjes, P.C.: Intracellular injections of lucifer yellow into lightly fixed mitral cells reveal neuronal dye-coupling in the developing rat olfactory bulb. Brain Res Dev Brain Res 84 (1): 1 - 10, 1995.

Pawley, J.B. (Editor): Handbook of biological confocal microscopy. Springer, $3^{\text {rd }}$ edition, 2006.

Pelz, C., Gerber, B., and Menzel, R.: Odorant intensity as a determinant for olfactory conditioning in honeybees: roles in discrimination, overshadowing and memory consolidation. J Exp Biol 200 (Pt 4): 837-847, 1997.

Petersen, R.S., Panzeri, S., and Diamond, M.E.: Population coding of stimulus location in rat somatosensory cortex. Neuron 32 (3): 503-514, 2001.

Petersen, R.S., Panzeri, S., and Diamond, M.E.: The role of individual spikes and spike patterns in population coding of stimulus location in rat somatosensory cortex. Biosystems 67 (1-3): 187 - 193, 2002.

Pinching, A.J. and Powell, T.P.: The neuropil of the glomeruli of the olfactory bulb. J Cell Sci 9 (2): 347 - 377, 1971.

Poher, V., Kennedy, G.T., Manning, H.B., Owen, D.M., Zhang, H.X., Gu, E., Dawson, M.D., French, P.M.W., and Neil, M.A.A.: Improved sectioning in a slit scanning confocal microscope. Opt Lett 33 (16): 1813 - 1815, 2008.

Rall, W., Shepherd, G.M., Reese, T.S., and Brightman, M.W.: Dendrodendritic synaptic pathway for inhibition in the olfactory bulb. Exp Neurol 14 (1): 44 $56,1966$.

Reddy, G.D. and Saggau, P.: Fast three-dimensional laser scanning scheme using acousto-optic deflectors. J Biomed Opt 10 (6): 064.038, 2005.

Reich, D.S., Mechler, F., and Victor, J.D.: Temporal coding of contrast in primary visual cortex: when, what, and why. J Neurophysiol 85 (3): 1039 - 1050, 2001. 
Ressler, K.J., Sullivan, S.L., and Buck, L.B.: Information coding in the olfactory system: evidence for a stereotyped and highly organized epitope map in the olfactory bulb. Cell 79 (7): 1245 - 1255, 1994.

Salin, P.A., Lledo, P.M., Vincent, J.D., and Charpak, S.: Dendritic glutamate autoreceptors modulate signal processing in rat mitral cells. J Neurophysiol 85 (3): $1275-1282,2001$.

Scheidweiler, U., Nezlin, L., Rabba, J., Müller, B., and Schild, D.: Slice culture of the olfactory bulb of Xenopus laevis tadpoles. Chem Senses 26 (4): 399 - 407, 2001.

Schild, D.: A computer-controlled device for the application of odours to aquatic animals. J Electrophysiol Techn 12: 71 - 79, 1985.

Schoppa, N.E., Kinzie, J.M., Sahara, Y., Segerson, T.P., and Westbrook, G.L.: Dendrodendritic inhibition in the olfactory bulb is driven by NMDA receptors. $J$ Neurosci 18 (17): 6790 - 6802, 1998.

Schoppa, N.E. and Westbrook, G.L.: Glomerulus-specific synchronization of mitral cells in the olfactory bulb. Neuron 31 (4): 639 - 651, 2001.

Schoppa, N.E. and Westbrook, G.L.: AMPA autoreceptors drive correlated spiking in olfactory bulb glomeruli. Nat Neurosci 5 (11): 1194 - 1202, 2002.

Scott, J.W.: Sniffing and spatiotemporal coding in olfaction. Chem Senses 31 (2): $119-130,2006$.

Shannon, C.: Communication in the presence of noise. Proceedings of the IRE $37(1): 10-21,1949$.

Sheppard, C.J.R. and Mao, X.Q.: Confocal microscopes with slit apertures. Journal of Modern Optics 35 (7): 1169 - 1185, 1988.

Shipley, M.T. and Ennis, M.: Functional organization of olfactory system. $J$ Neurobiol 30 (1): 123 - 176, 1996. 
Skarda, C.A. and Freeman, W.J.: How brains make chaos in order to make sense of the world. Behavioral And Brain Sciences 10: 161 - 195, 1987.

Slotnick, B.M., Graham, S., Laing, D.G., and Bell, G.A.: Detection of propionic acid vapor by rats with lesions of olfactory bulb areas associated with high 2-dg uptake. Brain Res 417 (2): 343 - 346, 1987.

Slotnick, B.: Odor-sampling time of mice under different conditions. Chem Senses 32 (5): $445-454,2007$ a.

Slotnick, B.: Response accuracy and odor sampling time in mice trained to discriminate between enantiomers of carvone and those of terpinen-4-ol. Chem Senses $32(7): 721-725,2007 \mathrm{~b}$.

Smetters, D., Majewska, A., and Yuste, R.: Detecting action potentials in neuronal populations with calcium imaging. Methods 18 (2): 215 - 221, 1999.

Smith, T.C. and Jahr, C.E.: Self-inhibition of olfactory bulb neurons. Nat Neurosci 5 (8): $760-766,2002$.

Spors, H. and Grinvald, A.: Spatio-temporal dynamics of odor representations in the mammalian olfactory bulb. Neuron 34 (2): 301 - 315, 2002.

Spors, H., Wachowiak, M., Cohen, L.B., and Friedrich, R.W.: Temporal dynamics and latency patterns of receptor neuron input to the olfactory bulb. J Neurosci 26 (4): $1247-1259,2006$.

Stocker, R.F.: Drosophila as a focus in olfactory research: mapping of olfactory sensilla by fine structure, odor specificity, odorant receptor expression, and central connectivity. Microsc Res Tech 55 (5): 284 - 296, 2001.

Stosiek, C., Garaschuk, O., Holthoff, K., and Konnerth, A.: In vivo two-photon calcium imaging of neuronal networks. Proc Natl Acad Sci USA 100 (12): 7319 $-7324,2003$.

Stuart, G., Spruston, N., Sakmann, B., and Häusser, M.: Action potential initiation and backpropagation in neurons of the mammalian CNS. Trends Neurosci 20 (3): $125-131,1997$. 
Tabor, R., Yaksi, E., Weislogel, J.M., and Friedrich, R.W.: Processing of odor mixtures in the zebrafish olfactory bulb. J Neurosci 24 (29): 6611 - 6620, 2004.

Takahashi, A., Camacho, P., Lechleiter, J.D., and Herman, B.: Measurement of intracellular calcium. Physiol Rev 79 (4): 1089-1125, 1999.

Tan, Y.P., Llano, I., Hopt, A., Würriehausen, F., and Neher, E.: Fast scanning and efficient photodetection in a simple two-photon microscope. $J$ Neurosci Methods 92 (1-2): 123 - 135, 1999.

Tietze, U. and Schenk, C.: Halbleiter-Schaltungstechnik. Springer, $11^{\text {th }}$ edition, 1999.

Tsien, R.Y.: A non-disruptive technique for loading calcium buffers and indicators into cells. Nature 290 (5806): $527-528,1981$.

Uchida, N. and Mainen, Z.F.: Speed and accuracy of olfactory discrimination in the rat. Nat Neurosci 6 (11): $1224-1229,2003$.

Urban, N.N.: Lateral inhibition in the olfactory bulb and in olfaction. Physiol Behav 77 (4-5): $607-612,2002$.

Usami, A., Matsuki, N., and Ikegaya, Y.: Spontaneous plasticity of multineuronal activity patterns in activated hippocampal networks. Neural Plast 2008: 108.969, 2008 .

Vassar, R., Chao, S.K., Sitcheran, R., NuÃśez, J.M., Vosshall, L.B., and Axel, R.: Topographic organization of sensory projections to the olfactory bulb. Cell $\mathbf{7 9}$ (6): $981-991,1994$.

Verhagen, J.V., Wesson, D.W., Netoff, T.I., White, J.A., and Wachowiak, M.: Sniffing controls an adaptive filter of sensory input to the olfactory bulb. Nat Neurosci 10 (5): $631-639,2007$.

Vucinić, D., Cohen, L.B., and Kosmidis, E.K.: Interglomerular center-surround inhibition shapes odorant-evoked input to the mouse olfactory bulb in vivo. $J$ Neurophysiol 95 (3): 1881 - 1887, 2006. 
Vucinić, D. and Sejnowski, T.J.: A compact multiphoton 3d imaging system for recording fast neuronal activity. PLoS ONE 2 (1): e699, 2007.

Waldman, B.: Olfactory basis of kin recognition in tadpoles. J Comp Physiol A 156: 565 - 577, 1985. Printed Copy Only.

Watterson, G.A., Ewens, W.J., and Hall, T.E.: The chromosome inversion problem. J. theor. Biol. 99: $1-7,1982$.

Weisstein, E.W.: CRC Concise Encyclopedia of Mathematics. Chapman \& Hall / CRC, $2^{\text {nd }}$ edition, 2002.

Wellis, D.P., Scott, J.W., and Harrison, T.A.: Discrimination among odorants by single neurons of the rat olfactory bulb. J Neurophysiol 61 (6): $1161-1177,1989$.

Wesson, D.W., Carey, R.M., Verhagen, J.V., and Wachowiak, M.: Rapid encoding and perception of novel odors in the rat. PLoS Biol 6 (4): e82, 2008.

Wilhelm, S., Gröbler, B., Gluch, M., and Heinz, H.: Principles of confocal laser scanning microscopy. Carl Zeiss, Inc., Jena, Germany, 2003.

Wilson, R.I., Turner, G.C., and Laurent, G.: Transformation of olfactory representations in the drosophila antennal lobe. Science 303 (5656): 366 - 370, 2004.

Yaksi, E., Judkewitz, B., and Friedrich, R.W.: Topological reorganization of odor representations in the olfactory bulb. PLoS Biol 5 (7): e178, 2007.

Yokoi, M., Mori, K., and Nakanishi, S.: Refinement of odor molecule tuning by dendrodendritic synaptic inhibition in the olfactory bulb. Proc Natl Acad Sci USA 92 (8): 3371 - 3375, 1995.

Youngentob, S.L., Markert, L.M., Mozell, M.M., and Hornung, D.E.: A method for establishing a five odorant identification confusion matrix task in rats. Physiol Behav 47 (6): 1053-1059, 1990.

Yu, C.R., Power, J., Barnea, G., O’Donnell, S., Brown, H.E.V., Osborne, J., Axel, R., and Gogos, J.A.: Spontaneous neural activity is required for the establishment and maintenance of the olfactory sensory map. Neuron 42 (4): 553-566, 2004. 


\section{Curriculum Vitæ}

\section{Personal Details}

Name

Date of birth

Place of birth

Address

Phone

E-mail

Graduate Studies

since 2005

\section{Academic Degree \\ Master's Thesis}

2005

\section{Undergraduate Studies}

$2003-2004$

$2002-2003$

2002

$2000-2002$

2000

$1997-2000$

High School

$1993-1996$

$1991-1993$
Stephan Junek

April 17 1977

Potsdam, Germany

Nikolaistr. 28, 37073 Göttingen, Germany

$+49-551-3919734$

sjunek1@gwdg.de

\section{Education}

International MSc/PhD/MD-PhD Program Neurosciences,

Department of Neurophysiology and Cellular Biophysics, University Göttingen, Supervisor: Prof. D. Schild

Master of Science

Intracellular $\mathrm{Ca}^{2+}$ recordings using glass fibers in olfactory cells Department of Neurophysiology and Cellular Biophysics, University Göttingen, Supervisor: Prof. D. Schild

International MSc/PhD/MD-PhD Program Neurosciences, Max-Planck-Research School for Neurosciences, Göttingen Study of biophysics, University of California in San Diego Intermediate Exams in Biophysics, Humboldt University Study of biophysics, Humboldt University Berlin Study of biology, Free University Berlin Study of music, 'Hochschule für Musik Berlin Hanns Eisler'

Gymnasium 'Hermann von Helmholtz' in Potsdam

'Weinberg Gymnasium' in Kleinmachnow 


\section{Scholarships}

$2005-2006$

$2003-2004$

$2002-2003$

2003

2003

2003

$2001-2002$

2001

$2005-2008$

2007

$2005-2007$

Lichtenberg Stipend

Max Planck Stipend

Humboldt - Stipend

\section{Laboratory Experiences}

Optical sectioning using widefield microscopy (Dept. of

Neurophysiology and Cellular Biophysics, University Göttingen)

Localization of raft related components during membrane remodelling

(Cell Biophysics Group, ENI Göttingen)

Birthdating of stix-positive cells in the neocortex and subiculum

(Dept. of Mol. Biol. of Neuronal Signals, MPI Experimental Medicine)

The Patch Clamp Guide (Dept. of Neurophysiology and

Cellular Biophysics, University Göttingen)

Student assistant, Fraunhofer Institut for Biomedical Engineering

Student assistant, Humboldt University Berlin

\section{Teaching Experiences}

2007 Supervision of the student project

Investigation of glomerular structure using fluorescent markers

Seminars and practical courses on biophysics

Practical courses on neurophysiology

Method courses on fluorescent and confocal microscopy

for the MPIRS for Neurosciences, Göttingen

\section{Courses and Workshops}

2005 Summer School for Computational Neuroscience, MPI for Dynamics and Self-organization, Göttingen

$20064^{\text {th }}$ European School of Neuro-IT and Neuroengineering, Genova. 


\section{Activities and Interests}

Organization

Music

Hobbies
Co-organizer of Neurizons 2007, an interdisciplanary meeting on the neurosciences, taking place May $31^{\text {st }}-$ June $2^{\text {nd }} 2007$ in the MPI for Experimental Medicine, Göttingen

Clarinetist, as soloist as well as in ensembles and orchestras 


\section{List of Publications}

Junek, S., Chen, T.W., Alevra, M., and Schild, D.: Activity Correlation Imaging: Visualizing Function and Structure of Neuronal Populations. Bioph J, accepted.

Olfactory coding with population onset times. In preparation.

Opazo, F., Junek, S., Schulz, J.B., and Falkenburger, B.H.: PKC Couples Metabotropic Receptors to Transporter-mediated Monoamine Efflux. Submitted.

Hassenklöver, T., Kurtanska, S., Bartoszek, I., Junek, S., Schild, D., and Manzini, I.: Nucleotide-induced $\mathrm{Ca}^{2+}$ signaling in sustentacular supporting cells of the olfactory epithelium. Glia 56 (15): 1614 - 1624, 2008.

Britanova, O., Alifragis, P., Junek, S., Jones, K., Gruss, P., and Tarabykin, V.: A novel mode of tangential migration of cortical projection neurons. Dev Biol. 298 (1): $299-311,2006$. 